WSRC-TR-2001-00125

Revision 0

Keywords: Coupled Operations DWPF, PCT, PCCS, Salt Disposition

Retention Time: Permanent

\title{
IMPACT OF COOLING RATE ON THE DURABILITY OF CST GLASSES: A NONPROPRIETARY SUMMARY (U)
}

T. B. Edwards

J. R. Harbour

R. J. Workman

Westinghouse Savannah River Company Savannah River Technology Center Aiken, SC 29808

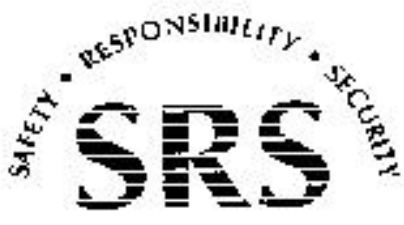

SAVANNAH RIYRII SITE

PREPARED FOR THE U.S. DEPARTMENT OF ENERGY UNDER CONTRACT NO. DE-AC09-96SR18500 
This document was prepared in conjunction with work accomplished under Contract No.

DE-AC09-96SR18500 with the U.S. Department of Energy.

\section{DISCLAIMER}

This report was prepared as an account of work sponsored by an agency of the United States Government. Neither the United States Government nor any agency thereof, nor any of their employees, makes any warranty, express or implied, or assumes any legal liability or responsibility for the accuracy, completeness, or usefulness of any information, apparatus, product or process disclosed, or represents that its use would not infringe privately owned rights. Reference herein to any specific commercial product, process or service by trade name, trademark, manufacturer, or otherwise does not necessarily constitute or imply its endorsement, recommendation, or favoring by the United States Government or any agency

thereof. The views and opinions of authors expressed herein do not necessarily state or reflect those of the United States Government or any agency thereof.

This report has been reproduced directly from the best available copy.

Available for sale to the public, in paper, from: U.S. Department of Commerce, National Technical Information Service, 5285 Port Royal Road, Springfield, VA 22161, phone: (800)

553-6847, fax: (703) 605-6900, email: orders@ntis.fedworld.gov online ordering: http://www.ntis.gov/ordering.htm

Available electronically at http://www.doe.gov/bridge

Available for a processing fee to U.S. Department of Energy and its contractors, in paper, from: U.S. Department of Energy, Office of Scientific and Technical Information, P.O. Box 62, Oak Ridge, TN 37831-0062, phone: (865 ) 576-8401, fax: (865) 576-5728, email: reports@ adonis.osti.gov 


\section{Impact of Cooling Rate on the Durability of CST Glasses: A Nonproprietary Summary (U)}

March 8, 2001

Document Approvals

T. B. Edwards, Author

Date

Statistical Consulting Section

J. R. Harbour, Author

Date

Immobilization Technology Section

R. J. Workman, Author

Date

Immobilization Technology Section

K. G. Brown, Technical Review

Date

Immobilization Technology Section

D. K. Peeler, Technical Review

Date

Immobilization Technology Section

R. C. Tuckfield Manager

Date

Statistical Consulting Section

R. H. Spires, Manager

Date

Immobilization Technology and Business Development Group

Immobilization Technology Section

Authorized Derivative Classifier

The Following Signatures Signify Satisfactory Completion of the FY01 CST Glass Study

H. H. Elder, Acting Manager, HLW Process Engineering

Date

J. T. Carter, Director, HLW Salt Waste Program Engineering

Date

D. W. Wester, TFA CST System Lead,

Date

Pacific Northwest National Laboratory 
WSRC-TR-2001-00125

Revision 0

This page intentionally left blank. 


\section{TABLE OF CONTENTS}

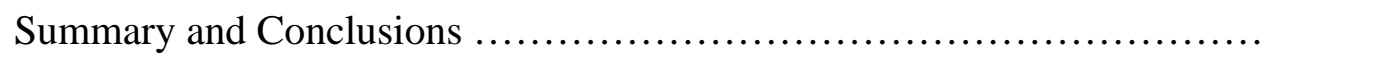

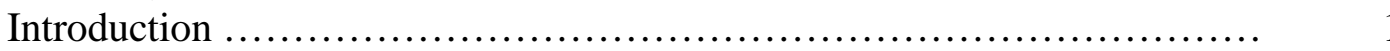

Results and Discussion ............................................ 3

Glass Batching and Cooling ................................... 3

Chemical Compositions ..................................... 4

PCT Results ................................................ 4

Quenched versus Centerline Cooled PCTs ....................... 9

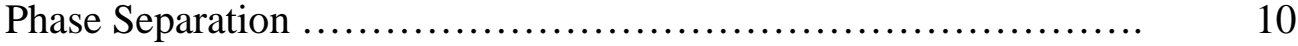

XRD and SEM Analysis of the Glasses........................... 11

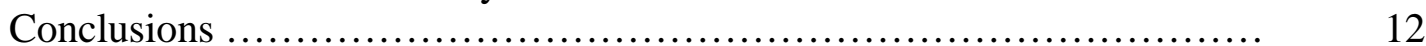

References ..................................................... 13

Appendix A. Supplemental Tables and Exhibits ......................... 15 
WSRC-TR-2001-00125

Revision 0

This page intentionally left blank. 


\section{SUMMARY AND CONCLUSION}

This report is a modified version of WSRC-TR-2001-00124, Revision 0. Information related to the chemical compositions of the study glasses has been removed from this version. WSRC has submitted a patent application for the frit (designated as BD1) utilized in the study and is limiting the distribution of the details of the compositions of the study glasses to protect its intellectual property rights pending patent clearance.

The crystalline silicotitanate (CST) glass study was conducted to determine the effect, if any, on the Product Consistency Test (PCT) responses of CST glasses cooled at different rates. The glasses contained CST and monosodium titanate (MST) [plus a simulated sludge representing Purex, Blend, or $\mathrm{HM}$ ] in amounts consistent with coupled operations using the CST option for salt disposition. Two bounding cooling profiles were used in this study: rapidly quenched and canister centerline cooled. Glasses were selected based on a number of criteria, but mainly to challenge the regions where amorphous phase separation might be expected. Currently, DWPF utilizes a homogeneity constraint to preclude glass regions that could lead to phase separation. However, this constraint was not developed for a compositional region that includes the levels of CST (3-9 wt\%) and MST (2.5 wt\%) introduced by this study. Due to this uncertainty, the CST glasses that were selected for study covered a wide range of values for the homogeneity constraint. Also, it was important to ensure that deleterious phase separation does not occur for either cooling profile. In this case, deleterious phase separation is defined as the formation of amorphous phase separation (or glass-in-glass) that significantly alters the glass durability as measured by the PCT response.

The durabilities, as measured by the PCT response of boron, for the CST glasses ranged from 0.41 to $0.80 \mathrm{~g} / \mathrm{L}$. These values are significantly lower than the PCT values for glasses containing precipitate hydrolysis aqueous (PHA) [as reported in WSRC-TR-2001-00123] and for environmental assessment (EA). The PCT results are highly clustered consistent with previous work. Many of the measured PCT values fall above the upper $95 \%$ prediction interval of the DWPF model used to predict durability, again, consistent with previous results. The very good durability of the CST containing glasses implies that durability may not be the limiting factor for waste loading in this option.

$\mathrm{X}$-ray diffraction (XRD) and scanning electron microscopy (SEM) were used to evaluate crystalline phases observed in the centerline cooled glasses. XRD analysis, which detects crystals throughout the bulk of the glass, revealed that trevorite and hematite were present in some of the glasses.

SEM analyses of the crystals that formed on the surface of the glasses revealed a variety of different species including titanium rods and spots, trevorite, trevorite enriched with titanium (which appears to correlate with the amount of titanium in the glass), and a phase rich in $\mathrm{Mn}$ and Fe. One glass, cst12, contained a crystal high in uranium content.

The results revealed that there was no practical difference between the PCT responses for glasses subjected to the two cooling profiles. In fact, although to a small extent, the boron PCT responses for the centerline-cooled glasses were more durable than the rapidly quenched glasses. These results reveal that no deleterious amorphous phase separation or crystalline phase occurred under either cooling regime for the glass compositions tested.

\section{INTRODUCTION}

One of the Alternative Salt Disposition Flowsheets being considered would require that the Defense Waste Processing Facility (DWPF) vitrify a coupled feed containing High Level Waste (HLW) and crystalline silicotitanate (CST). A Technical Task Request (TTR) [1] was received as part of the Salt Processing Program by the Savannah River Technology Center (SRTC) requesting that a glass variability study be conducted to explore the impact of cooling rate on glass durability for this alternative. A Task Technical and Quality Assurance (TT\&QA) plan [2] was issued by SRTC in response to the TTR. The objective of this task is to study the impact of cooling rate on the durability 
WSRC-TR-2001-00125

Revision 0

of glass with anticipated levels of CST in DWPF glasses with nominal levels of monosodium titanate (MST).

The set of target glass compositions for this task was provided in WSRC-TR-2001-00124, Revision 0. The study glasses were selected from a set of candidate glasses that covered Purex, HM, and Blend sludge types at loadings (in the glass) of 22 to 30 oxide weight percent (wt $\%$ ), utilized CST loadings (in the glass) of 3,6, and 9 oxide wt $\%$, and included MST concentrations (in the glass) mainly at 2.5 wt $\%$. A $\pm 10 \%$ variation in the composition of each sludge type was also utilized in the development of the set of candidate glass compositions. For each composition, the remainder of the glass consisted of glass formers collectively referred to as $\mathrm{BD} 1^{1}$, the composition of which is WSRC proprietary information.

Compositions were selected from the set of candidate points that increased the opportunity of seeing any significant cooling impact on glass durability as measured by the PCT. Several glasses were chosen that would also demonstrate reproducibility of the results from an earlier study [3]. The general targeted compositions for the CST glasses are provided in Table 1.

Table 1: Targeted Compositions (in wt \%) for the CST FY01 Study

\begin{tabular}{|c|ccccc|}
\hline Glass & Sludge & & & & BD1 \\
ID & Type & Sludge & MST & CST & Frit \\
cst01 & Purex & 26 & 2.50 & 9 & 62.50 \\
cst02 & HM & 26 & 1.25 & 6 & 66.75 \\
cst03 & Purex & 22 & 2.50 & 9 & 66.50 \\
cst04 & Purex & 30 & 1.25 & 6 & 62.75 \\
cst05 & Purex & 26 & 2.50 & 3 & 68.50 \\
cst06 & Purex & 30 & 1.25 & 9 & 59.75 \\
cst07 & Purex & 22 & 1.25 & 3 & 73.75 \\
cst08 & Purex & 30 & 2.50 & 6 & 61.50 \\
cst09 & Purex & 26 & 1.25 & 9 & 63.75 \\
cst10 & Blend & 30 & 2.50 & 6 & 61.50 \\
cst11 & Blend & 28 & 2.50 & 9 & 60.50 \\
cst12 & Blend & 30 & 1.25 & 3 & 65.75 \\
cst13 & Blend & 26 & 1.25 & 6 & 66.75 \\
cst14 & HM & 26 & 1.25 & 6 & 66.75 \\
cst15 & Blend & 26 & 1.25 & 6 & 66.75 \\
cst16 & HM & 26 & 1.25 & 6 & 66.75 \\
cst17 & Blend & 26 & 1.25 & 6 & 66.75 \\
\hline
\end{tabular}

Property models [4] that are utilized by the DWPF for process and product quality control were used to help in the selection of the study glasses. The process properties of interest included viscosity and liquidus temperature. The product property of interest for the study glasses is durability (as measured by the 7-day Product Consistency Test (PCT) [5]). Currently, DWPF utilizes a homogeneity constraint to preclude glass regions that could lead to amorphous phase separation. However, this constraint was not developed for a compositional region that includes the levels of CST (3-9 wt\%) and MST (2.5 wt\%) introduced by this study [6]. Due to this uncertainty, the CST glasses that were selected for study covered a wide range of values for the homogeneity constraint. These property predictions along with other key characteristics of the targeted compositions are provided in Table 2.

1 A patent application has been submitted for the formulation of BD1, and, thus, the details associated with the chemical compositions of the study glasses are not provided in this report. 
WSRC-TR-2001-00125

Revision 0

Table 2: Property Predictions for the CST Study Glasses

\begin{tabular}{|c|c|c|c|c|c|c|c|c|c|c|c|c|c|c|}
\hline \multirow[b]{2}{*}{ Glass } & \multirow[b]{2}{*}{ Sludge } & & & & & & & & & \multicolumn{3}{|c|}{$\begin{array}{c}\text { Normalized } \\
\text { Releases }\end{array}$} & \multirow[b]{2}{*}{ Visc. } & \multirow[b]{2}{*}{$\mathbf{T}_{\mathrm{L}}$} \\
\hline & & \multicolumn{4}{|c|}{ Loadings } & $\mathrm{Al}_{2} \mathrm{O}_{3}$ & alkalis & Homo & & B & $\mathbf{L i}$ & $\mathbf{N a}$ & & \\
\hline ID & Type & $\mathbf{S}$ & MST & CST & Frit & $(\mathrm{wt} \%)$ & (wt\%) & $(w t \%)$ & del Gp & $(\mathrm{g} / \mathrm{L})$ & $(\mathrm{g} / \mathrm{L})$ & $(g / L)$ & (P) & ${ }^{\mathbf{0}} \mathrm{C}$ \\
\hline cst01 & Purex & 26 & 2.50 & 9 & 62.50 & 2.870 & 13.12 & 197.9 & -6.3390 & 0.18 & 0.24 & 0.19 & 72.8 & 994.8 \\
\hline cst02 & & 26 & 1.25 & 6 & 66.75 & 7.060 & & & & & 0.17 & 0.13 & 162.2 & 926.3 \\
\hline st03 & & 22 & 2.50 & 9 & 66.50 & 2.720 & & & 769 & 0.20 & 0.27 & 0.22 & 89.6 & 939.8 \\
\hline st 04 & & 30 & 1.25 & - & 62.75 & 3.550 & & & & 077 & 0.34 & .28 & 67.9 & 1007.3 \\
\hline cst05 & & 26 & & & & & & & & & 0.35 & 29 & 8 & 983.1 \\
\hline cst06 & $\mathrm{ex}$ & 30 & 1.25 & 9 & 59.75 & 3.530 & 46 & 209.7 & 54 & 0.20 & 0.27 & 0.22 & 60.7 & 1038.5 \\
\hline cst07 & & 22 & 1.25 & 3 & 73.75 & 2.340 & 49 & 203.3 & & 0.25 & 0.33 & 0.27 & 88.2 & 955.7 \\
\hline cst08 & & 30 & 2.50 & 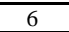 & 61.50 & 2.960 & & & & & 0.24 & 0.19 & 58.5 & 1054.2 \\
\hline cst09 & & 26 & 1.25 & 9 & 63.75 & 2.630 & & .5 & & & 0.25 & 0.20 & 78.2 & 986.7 \\
\hline cst10 & d & 30 & 2.50 & 7 & 61.50 & \begin{tabular}{|l|}
4.440 \\
\end{tabular} & 78 & 208.9 & 90 & 0 & 0.16 & 0.12 & 105.1 & 1009.8 \\
\hline cst11 & end & 28 & 2.50 & 9 & 60.50 & 4.290 & 28 & .8 & 571 & 0.07 & 0.12 & 0.08 & 103.8 & 1023.5 \\
\hline cst12 & & 30 & 1.25 & 3 & 65.75 & 4.600 & & & 887 & 0.10 & 0.16 & 0.12 & 103.9 & 1026.8 \\
\hline cst13 & & 26 & & 6 & & 4.3 & & & & & 0.25 & 0.20 & 94.1 & 978.3 \\
\hline cst14 & & 26 & & 6 & 66.75 & 7.060 & & 204.9 & & & 0.28 & 0.23 & 120.9 & 931.1 \\
\hline $\operatorname{cst} 15$ & & 26 & 1.25 & 6 & 66.75 & 4.320 & 13.57 & 206.5 & & 0.21 & 0.28 & 0.22 & 88.3 & 979.6 \\
\hline cst16 & & 26 & 1.25 & 6 & 66.75 & 7.060 & & & & 0.31 & 0.39 & 0.33 & 100.5 & 934.1 \\
\hline cst17 & Blend & 26 & 1.25 & 6 & 66.75 & 4.320 & 14.57 & 206.5 & -7.6419 & 0.30 & 0.38 & 0.32 & 72.5 & 983.6 \\
\hline
\end{tabular}

The purpose of this report is to provide and investigate comparisons between

- $\quad$ the measured and target compositions of the CST glasses,

- $\quad$ the PCT measurements and durability model predictions, and

- $\quad$ the PCT measurements for quenched versus centerline-cooled glasses.

Evaluating the results from these comparisons provides the basis for assessing the impact of cooling rate on the durability of CST glasses.

\section{RESULTS AND DISCUSSION}

The 17 glasses comprising the CST study were designated as cst01 through cst17. Composition and PCT measurements of these glasses were conducted in accordance with analytical plans that were used to generate the measurements supporting the CST study. (See WSRC-TR-2001-00124, Revision 0.) These plans were prepared to support the overall Task Technical and QA plan [2] and the analytical study plan [7]. The results of the composition measurements were provided in WSRC-TR-200100124, Revision 0 and are not discussed in this report.

Predictions for the properties of interest that were generated for these target compositions by the models utilized by the DWPF were also included in WSRC-TR-2001-00124, Revision 0. These propertiesrelate a given composition to its processability and product quality. For a given composition, acceptable property characteristics and reliable property predictions (using the current DWPF models) are of interest. The critical property for this study is glass durability. Comparisons between predictions and measurements for this property are provided for both cooling treatments in the discussion that follows.

\section{Glass Batching and Cooling}

Glasses were batched and fabricated to targeted compositions and were identified by cst01 though cst17 as indicated in Table 1. Since the compositions of the simulated sludges were allowed to vary $\pm 10 \%$ around their respective nominal compositions, their contributions to the CST glasses had to be represented as dry chemicals (e.g., reagent grade nitrates, carbonates, and oxides). The contributions of the glass formers (as BD1) to the composition of the study glasses were introduced the same way. The contribution of the MST was represented by appropriate addition of this material in the batching of each glass.

For each glass, the combined powders ( 120 grams) were added to a $100 \mathrm{~mL} \mathrm{Pt}-\mathrm{Au}$ crucible and placed in a calibrated furnace that was heated to $1150^{\circ} \mathrm{C}$ at a rate of $10^{\circ} \mathrm{C} /$ minute and then held for four hours at $1150^{\circ} \mathrm{C}$. Then the crucible was removed and the glass was immediately poured onto a clean stainless steel plate, resulting in what is referred to as a "quenched" glass. 
A portion of each glass cooled by quenching was then remelted and allowed to cool following a centerline cooling profile. This was accomplished by taking a portion of each glass and placing it in a smaller crucible. Then, the crucible was introduced to a furnace at $1150^{\circ} \mathrm{C}$. The furnace was programmed to cool at a rate that simulated the centerline cooling profile of DWPF canisters.

\section{Chemical Compositions}

In addition to the CST glasses, a standard glass (Batch 1) and a standard uranium-bearing glass were included in the planning of these analyses. These standards were used to correct for potential biases in the measured chemical compositions of the study glasses. Once again, a detailed discussion of the chemical compositions is provided in WSRC-TR-2001-00124, Revision 0. However, in the discussion that follows three options for the compositions of the glasses [targeted, measured, and measured bias corrected (bc)] are provided.

\section{PCT Results}

Samples of the 17 CST glasses, after being batched and fabricated (via quenching), were subjected to a second heat treatment. They were cooled to simulate a canister-centerline-cooling profile. Differences in glass durability for these two cooling regimes (quenched versus centerline cooled) are of primary interest to this study. The investigation into this question required durability to be measured for the quenched and centerline cooled versions of each of the study glasses.

The 7-day Product Consistency Test (PCT) was used as the assessment of glass durability [5]. More specifically, Method A of the PCT (ASTM C1285) was used for these measurements. The PCTs were conducted in triplicate for the CST glasses. In addition, PCTs were also conducted in triplicate for samples of the Environmental Assessment (EA) glass, the Approved Reference Material (ARM) glass, and a blank (ASTM Type I water). An analytical plan (see WSRC-TR-2001-00124, Revision 0) supporting these tests was provided to assist the SRTC-ML in measuring the compositions of the solutions resulting from these PCTs. Of primary interest were the concentrations (in parts per million, $\mathrm{ppm}$ ) of boron (B), lithium ( $\mathrm{Li})$, sodium $(\mathrm{Na})$, and silicon $(\mathrm{Si})$. Samples of a multi-element solution standard were also included in the analytical plan (as a check on the accuracy of the inductively coupled plasma (ICP) - emission spectrometer used for these measurements).

The results from these tests are given in Table A.1 of Appendix A. The PCT results for the centerlinecooled version of each CST study glass are indicated by the "clc" suffix on the glass ID. One of the quality control checkpoints for the PCT procedure is solution-weight loss over the course of the 7-day test. The shaded entries of Table A3 indicate those solutions that fell outside the weight-loss guidelines (weight loss must be less than 5\%). Two successful solutions out of the 3 conducted for a glass are required to generate a representative PCT for that glass [5]. Although this criterion is not met for all of the CST study glasses, the results are believed to provide meaningful and representative comparisons for assessing the impact of the cooling regimes.

Any measurement in the "as reported" columns of Table A.3 proceeded by a "<" was below the detection limit for this measurement. The measurement was replaced by one half of the detection limit in the determination of the parts per million (ppm) columns of the table. The values in the ppm columns were also adjusted for the dilution factors by multiplying the "as-reported" values by 1.6667 for the CST and ARM glasses and by 16.6667 for the EA glass. Thus, the concentrations in the ppm columns reflect detection and dilution adjustments.

Exhibit A.1 in the Appendix provides pairs of plots of the leachate concentrations and standards in the analytical sequence reported by the SRTC-ML. For each element of interest, one plot of the pair includes the values from the EA PCTs and the blanks. These values expand the scales of these plots, making it difficult to distinguish among the results of the other analyses. The other plot excludes the EA and blank results. 
WSRC-TR-2001-00125

Revision 0

Exhibit A.2 in the Appendix provides pairs of plots of the leachate concentrations for each type of submitted solution: the standards, the blanks, EA, ARM, and the CST glasses. One plot of the pair includes EA and the blank. The other does not.

PCT leachate concentrations are typically normalized using the cation composition (expressed as a weight percent) in the glass to obtain a grams-per-liter $(\mathrm{g} / \mathrm{L})$ leachate concentration. The normalization of the PCTs is usually conducted using the measured compositions of the glasses. This is the preferred normalization process for the PCTs. For completeness, the targeted cation and the bias-corrected cation compositions are also used to conduct this normalization.

As is the usual convention, the common logarithm of the normalized PCT (normalized leachate, NL) for each element of interest are determined and used for comparison. To accomplish this computation, one must

1. Determine the common logarithm of the elemental parts per million (ppm) leachate concentration for each of the triplicates and each of the elements of interest (these values are provided in Table A.3 of Appendix A),

2. Average the common logarithms over the triplicates for each element of interest, and then

Normalizing Using Measured Composition (preferred method)

3. Subtract a quantity equal to 1 plus the common logarithm of the average cation measured concentration (expressed as a weight percent of the glass) from the average computed in step 2.

Or Normalizing Using Target Composition

3. Subtract a quantity equal to 1 plus the common logarithm of the target cation concentration (expressed as a weight percent of the glass) from the average computed in step 2 .

Or Normalizing Using Measured Bias-Corrected Composition

3. Subtract a quantity equal to 1 plus the common logarithm of the measured bias-corrected cation concentration (expressed as a weight percent of the glass) from the average computed in step 2.

As a preliminary step to completing these normalizations of the PCTs, statistical analyses were conducted on the three measurements of the multi-element standard solution per analytical block. Exhibit A.3 in the Appendix provides these analyses. Although there appears to be statistical differences among the block averages, no bias correction of the PCT results for the study glasses was conducted. This approach was taken since the triplicate PCTs for a single study glass were placed in different ICP blocks. Averaging the ppm's for each set of triplicates helps to minimize the impact of the ICP effects.

The block averages are presented in Table 3 . They indicate consistent and reasonably accurate results (differences of overall averages versus reference values $<5 \%$ ) from these analyses.

Table 3: Measurements of Standard Solution

\begin{tabular}{|c|c|c|c|c|}
\hline Analytical & Avg & Avg & Avg & Avg \\
\hline Block & B (ppm) & Li (ppm) & Na (ppm) & Si (ppm) \\
\hline 1 & 19.6 & 9.5 & 82.6 & 49.7 \\
\hline 2 & 19.3 & 9.3 & 77.8 & 49.8 \\
\hline 3 & 20.1 & 9.9 & 78.6 & 50.2 \\
\hline 4 & 19.6 & 10.4 & 80.5 & 48.5 \\
\hline 5 & 19.3 & 9.5 & 81.4 & 50.1 \\
\hline 6 & 20.0 & 10.1 & 80.7 & 51.2 \\
\hline Grand Average & 19.6 & 9.8 & 80.3 & 49.9 \\
\hline Reference Value & 20 & 10 & 81 & 50 \\
\hline \% difference & $-1.8 \%$ & $-2.2 \%$ & $-0.9 \%$ & $-0.2 \%$ \\
\hline
\end{tabular}


Table 4 provides the results from the normalization process using the information on the chemical compositions of the glasses (see WSRC-TR-2001-00124, Revision 0) and all of the data of Table A.1 (i.e., before screening the PCT results for solution weight problems). Exhibit A.4 in Appendix A provides scatter plots for these results (both quenched and centerline-cooled) and offers an opportunity to investigate the consistency in the leaching across the elements for the glasses of this study. The consistency is typically demonstrated by a high degree of linear correlation among the values. PCT values normalized using targeted, measured, and bias-corrected compositions are investigated. A high degree of correlation is not seen for these data for many pairs of the elements. The largest correlation $(\sim 81 \%)$ is between $\mathrm{B}$ and $\mathrm{Li}$ for the unscreened PCTs normalized using the targeted compositions. The smallest correlation $(\sim 38 \%)$ is between $\mathrm{Li}$ and $\mathrm{Na}$. These poor correlations may be due in part to the relative insensitivity of the PCT responses to the compositional variations of the study glasses. For example, all of the $\log \mathrm{NL}[\mathrm{B}(\mathrm{g} / \mathrm{L})]$ values of Table 4 fall within the interval $(-0.382,-0.099)$ for the CST study glasses.

Table 4: Normalized PCTs before Screening for Solution-Weight Problems

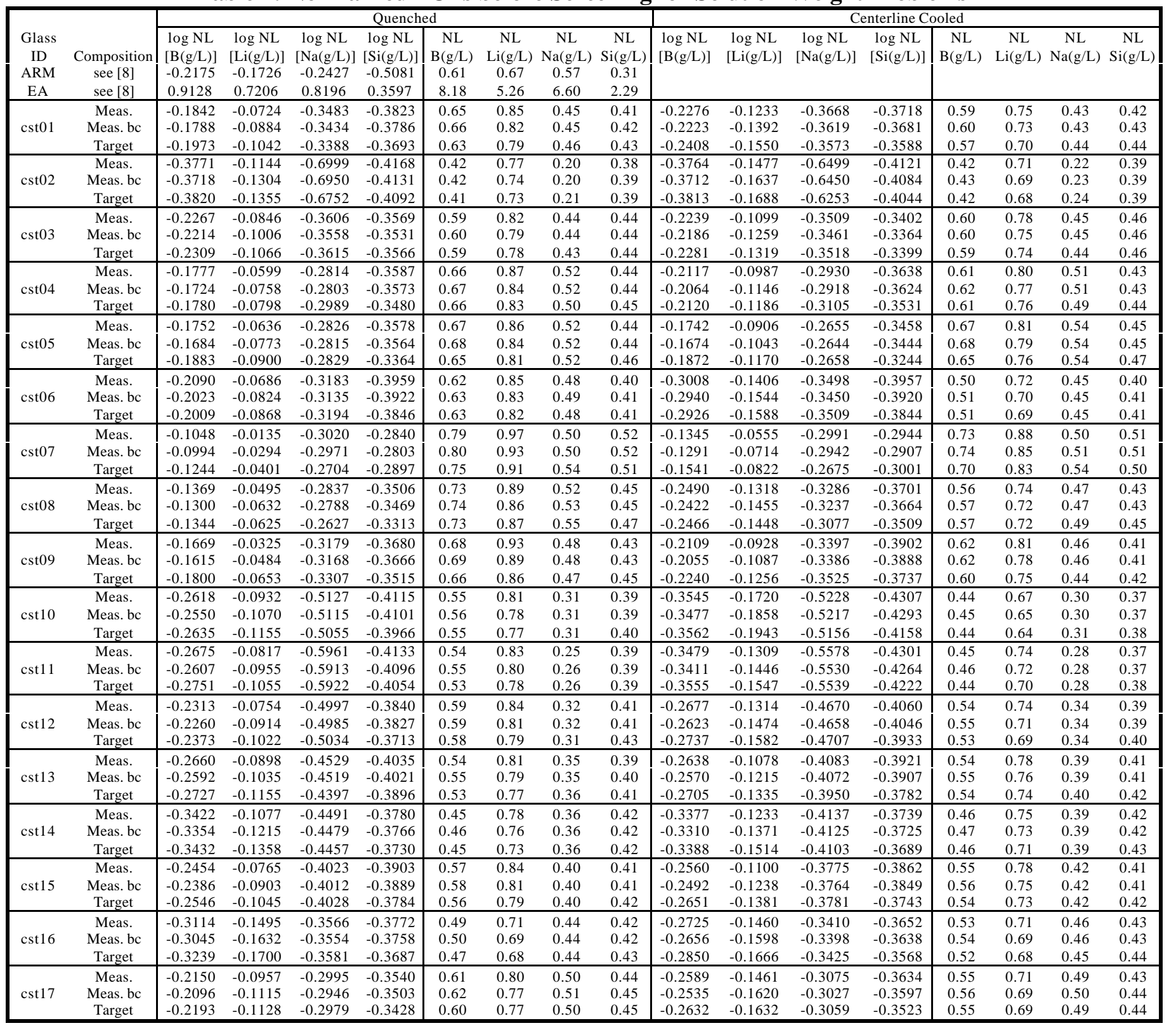


Table 5 provides the results from the normalization process using the information on the chemical compositions of the glasses and the screened data of Table A.1 (i.e., after screening the PCT results for solution weight problems). A valid PCT [5] for a glass requires that at least 2 of the 3 triplicates pass the solution-weight criterion (a weight loss of less than 5\%). The glasses whose PCT results do not satisfy that criterion are shaded in Table 5. All of the other entries of Table 5 are deemed valid PCTs. Exhibit A.5 in Appendix A provides correlations and scatter plots for the screened results (including both quenched and centerline-cooled PCTs) and offers an opportunity to investigate the consistency in the leaching across the elements for the glasses of this study. Correlations and scatter plots of just the valid PCTs are also provided as part of this exhibit. The consistency is typically demonstrated by a high degree of linear correlation among the values. PCT normalized using targeted, measured, and bias-corrected compositions are investigated. A high degree of correlation is not seen for these data for many pairs of the elements. The largest correlation is only $\sim 82 \%$; the smallest, only slightly over $27 \%$.

Table 5: Normalized PCTs after Screening for Solution-Weight Problems ${ }^{2}$

\begin{tabular}{|c|c|c|c|c|c|c|c|c|c|c|c|c|c|c|c|c|c|}
\hline \multirow[b]{2}{*}{$\begin{array}{l}\text { Glass } \\
\text { ID } \\
\text { ARM } \\
\text { EA } \\
\end{array}$} & \multirow[b]{2}{*}{$\begin{array}{c}\text { Composition } \\
\text { see [8] } \\
\text { see [8] } \\
\end{array}$} & \multicolumn{8}{|c|}{ Quenched } & \multicolumn{8}{|c|}{ Centerline Cooled } \\
\hline & & $\begin{array}{c}\log \mathrm{NL} \\
{[\mathrm{B}(\mathrm{g} / \mathrm{L})]} \\
-0.2175 \\
0.9128\end{array}$ & $\begin{array}{c}\log \mathrm{NL} \\
{[\mathrm{Si}(\mathrm{g} / \mathrm{L})]} \\
-0.1726 \\
0.7206\end{array}$ & $\begin{array}{c}\log \mathrm{NL} \\
{[\mathrm{Na}(\mathrm{g} / \mathrm{L})]} \\
-0.2427 \\
0.8196\end{array}$ & $\begin{array}{c}\log \mathrm{NL} \\
{[\mathrm{Li}(\mathrm{g} / \mathrm{L})]} \\
-0.5081 \\
0.3597\end{array}$ & $\begin{array}{c}\mathrm{NL} \\
\mathrm{B}(\mathrm{g} / \mathrm{L}) \\
0.61 \\
8.18\end{array}$ & $\begin{array}{c}\mathrm{NL} \\
\mathrm{Si}(\mathrm{g} / \mathrm{L}) \\
0.67 \\
5.26\end{array}$ & $\begin{array}{c}\mathrm{NL} \\
\mathrm{Na}(\mathrm{g} / \mathrm{L}) \\
0.57 \\
6.60\end{array}$ & $\begin{array}{c}\mathrm{NL} \\
\mathrm{Li}(\mathrm{g} / \mathrm{L}) \\
0.31 \\
2.29\end{array}$ & $\begin{array}{c}\log \mathrm{NL} \\
{[\mathrm{B}(\mathrm{g} / \mathrm{L})]}\end{array}$ & $\begin{array}{c}\log \mathrm{NL} \\
{[\mathrm{Si}(\mathrm{g} / \mathrm{L})]}\end{array}$ & $\begin{array}{c}\log \mathrm{NL} \\
{[\mathrm{Na}(\mathrm{g} / \mathrm{L})]}\end{array}$ & $\begin{array}{c}\log \mathrm{NL} \\
{[\mathrm{Li}(\mathrm{g} / \mathrm{L})]}\end{array}$ & $\begin{array}{c}\mathrm{NL} \\
\mathrm{B}(\mathrm{g} / \mathrm{L})\end{array}$ & $\begin{array}{c}\mathrm{NL} \\
\mathrm{Si}(\mathrm{g} / \mathrm{L})\end{array}$ & $\begin{array}{c}\mathrm{NL} \\
\mathrm{Na}(\mathrm{g} / \mathrm{L})\end{array}$ & $\begin{array}{c}\mathrm{NL} \\
\mathrm{Li}(\mathrm{g} / \mathrm{L})\end{array}$ \\
\hline $\operatorname{cst} 01$ & $\begin{array}{l}\text { Meas. } \\
\text { Meas. bc } \\
\text { Target }\end{array}$ & $\begin{array}{l}-0.1915 \\
-0.1862 \\
-0.2047 \\
\end{array}$ & $\begin{array}{l}-0.0747 \\
-0.0907 \\
-0.1065 \\
\end{array}$ & $\begin{array}{l}-0.3547 \\
-0.3498 \\
-0.3452 \\
\end{array}$ & $\begin{array}{l}-0.3912 \\
-0.3874 \\
-0.3781 \\
\end{array}$ & $\begin{array}{l}0.64 \\
0.65 \\
0.62\end{array}$ & $\begin{array}{l}0.84 \\
0.81 \\
0.78\end{array}$ & $\begin{array}{l}0.44 \\
0.45 \\
0.45\end{array}$ & $\begin{array}{l}0.41 \\
0.41 \\
0.42\end{array}$ & $\begin{array}{l}-0.2276 \\
-0.2223 \\
-0.2408 \\
\end{array}$ & $\begin{array}{l}-0.1233 \\
-0.1392 \\
-0.1550 \\
\end{array}$ & $\begin{array}{l}-0.3668 \\
-0.3619 \\
-0.3573 \\
\end{array}$ & $\begin{array}{l}-0.3718 \\
-0.3681 \\
-0.3588 \\
\end{array}$ & $\begin{array}{l}0.59 \\
0.60 \\
0.57\end{array}$ & $\begin{array}{l}0.75 \\
0.73 \\
0.70\end{array}$ & $\begin{array}{l}0.43 \\
0.43 \\
0.44 \\
\end{array}$ & $\begin{array}{l}0.42 \\
0.43 \\
0.44 \\
\end{array}$ \\
\hline cst02 & $\begin{array}{l}\text { Meas. } \\
\text { Meas. bc } \\
\text { Target }\end{array}$ & $\begin{array}{l}-0.3771 \\
-0.3718 \\
-0.3820 \\
\end{array}$ & $\begin{array}{l}-0.1144 \\
-0.1304 \\
-0.1355 \\
\end{array}$ & $\begin{array}{l}-0.6999 \\
-0.6950 \\
-0.6752 \\
\end{array}$ & $\begin{array}{l}-0.4168 \\
-0.4131 \\
-0.4092 \\
\end{array}$ & $\begin{array}{l}0.42 \\
0.42 \\
0.41 \\
\end{array}$ & $\begin{array}{l}0.77 \\
0.74 \\
0.73 \\
\end{array}$ & $\begin{array}{l}0.20 \\
0.20 \\
0.21 \\
\end{array}$ & $\begin{array}{l}0.38 \\
0.39 \\
0.39 \\
\end{array}$ & $\begin{array}{l}-0.3335 \\
-0.3283 \\
-0.3384 \\
\end{array}$ & $\begin{array}{l}-0.1028 \\
-0.1187 \\
-0.1239 \\
\end{array}$ & $\begin{array}{l}-0.5648 \\
-0.5599 \\
-0.5402 \\
\end{array}$ & $\begin{array}{l}-0.4080 \\
-0.4042 \\
-0.4003 \\
\end{array}$ & $\begin{array}{l}0.46 \\
0.47 \\
0.46 \\
\end{array}$ & $\begin{array}{l}0.79 \\
0.76 \\
0.75 \\
\end{array}$ & $\begin{array}{l}0.27 \\
0.28 \\
0.29 \\
\end{array}$ & $\begin{array}{l}0.39 \\
0.39 \\
0.40 \\
\end{array}$ \\
\hline $\operatorname{cst} 03$ & $\begin{array}{l}\text { Meas. } \\
\text { Meas. bc } \\
\text { Target }\end{array}$ & $\begin{array}{l}-0.2472 \\
-0.2419 \\
-0.2514 \\
\end{array}$ & $\begin{array}{l}-0.0996 \\
-0.1156 \\
-0.1216 \\
\end{array}$ & $\begin{array}{l}-0.3542 \\
-0.3494 \\
-0.3551 \\
\end{array}$ & $\begin{array}{l}-0.3730 \\
-0.3693 \\
-0.3728 \\
\end{array}$ & $\begin{array}{l}0.57 \\
0.57 \\
0.56 \\
\end{array}$ & $\begin{array}{l}0.79 \\
0.77 \\
0.76 \\
\end{array}$ & $\begin{array}{l}0.44 \\
0.45 \\
0.44 \\
\end{array}$ & $\begin{array}{l}0.42 \\
0.43 \\
0.42\end{array}$ & $\begin{array}{l}-0.2484 \\
-0.2431 \\
-0.2525 \\
\end{array}$ & $\begin{array}{l}-0.1196 \\
-0.1355 \\
-0.1416 \\
\end{array}$ & $\begin{array}{l}-0.3509 \\
-0.3461 \\
-0.3518 \\
\end{array}$ & $\begin{array}{l}-0.3540 \\
-0.3503 \\
-0.3537 \\
\end{array}$ & $\begin{array}{l}0.56 \\
0.57 \\
0.56 \\
\end{array}$ & $\begin{array}{l}0.76 \\
0.73 \\
0.72 \\
\end{array}$ & $\begin{array}{l}0.45 \\
0.45 \\
0.44 \\
\end{array}$ & $\begin{array}{l}0.44 \\
0.45 \\
0.44 \\
\end{array}$ \\
\hline $\operatorname{cst} 04$ & $\begin{array}{c}\text { Meas. } \\
\text { Meas. bc } \\
\text { Target }\end{array}$ & & & . & . & & & & & $\begin{array}{l}-0.2117 \\
-0.2064 \\
-0.2120 \\
\end{array}$ & $\begin{array}{l}-0.0987 \\
-0.1146 \\
-0.1186 \\
\end{array}$ & $\begin{array}{l}-0.2930 \\
-0.2918 \\
-0.3105 \\
\end{array}$ & \begin{tabular}{l|}
-0.3638 \\
-0.3624 \\
-0.3531 \\
\end{tabular} & $\begin{array}{l}0.61 \\
0.62 \\
0.61 \\
\end{array}$ & $\begin{array}{l}0.80 \\
0.77 \\
0.76 \\
\end{array}$ & $\begin{array}{l}0.51 \\
0.51 \\
0.49 \\
\end{array}$ & $\begin{array}{l}0.43 \\
0.43 \\
0.44 \\
\end{array}$ \\
\hline $\operatorname{cst} 05$ & $\begin{array}{l}\text { Meas. } \\
\text { Meas. bc } \\
\text { Target }\end{array}$ & $\begin{array}{r}-0.1682 \\
-0.1614 \\
-0.1812 \\
\end{array}$ & $\begin{array}{l}-0.0546 \\
-0.0683 \\
-0.0810 \\
\end{array}$ & $\begin{array}{r}-0.2785 \\
-0.2773 \\
-0.2787 \\
\end{array}$ & $\begin{array}{l}-0.3645 \\
-0.3631 \\
-0.3431 \\
\end{array}$ & $\begin{array}{l}0.68 \\
0.69 \\
0.66 \\
\end{array}$ & $\begin{array}{l}0.88 \\
0.85 \\
0.83 \\
\end{array}$ & $\begin{array}{l}0.53 \\
0.53 \\
0.53 \\
\end{array}$ & $\begin{array}{l}0.43 \\
0.43 \\
0.45 \\
\end{array}$ & $\begin{array}{l}-0.1742 \\
-0.1674 \\
-0.1872 \\
\end{array}$ & $\begin{array}{l}-0.0906 \\
-0.1043 \\
-0.1170 \\
\end{array}$ & $\begin{array}{l}-0.2655 \\
-0.2644 \\
-0.2658 \\
\end{array}$ & $\begin{array}{l}-0.3458 \\
-0.3444 \\
-0.3244 \\
\end{array}$ & $\begin{array}{l}0.67 \\
0.68 \\
0.65 \\
\end{array}$ & $\begin{array}{l}0.81 \\
0.79 \\
0.76 \\
\end{array}$ & $\begin{array}{l}0.54 \\
0.54 \\
0.54 \\
\end{array}$ & $\begin{array}{l}0.45 \\
0.45 \\
0.47 \\
\end{array}$ \\
\hline cst06 & $\begin{array}{l}\text { Meas. } \\
\text { Meas. bc } \\
\text { Target }\end{array}$ & $\begin{array}{l}-0.2309 \\
-0.2242 \\
-0.2228 \\
\end{array}$ & $\begin{array}{l}-0.1023 \\
-0.1161 \\
-0.1205\end{array}$ & $\begin{array}{l}-0.3551 \\
-0.3503 \\
-0.3563 \\
\end{array}$ & $\begin{array}{l}-0.3946 \\
-0.3908 \\
-0.3832 \\
\end{array}$ & $\begin{array}{l}0.59 \\
0.60 \\
0.60\end{array}$ & $\begin{array}{l}0.79 \\
0.77 \\
0.76\end{array}$ & $\begin{array}{l}0.44 \\
0.45 \\
0.44\end{array}$ & $\begin{array}{l}0.40 \\
0.41 \\
0.41\end{array}$ & $\begin{array}{l}-0.3008 \\
-0.2940 \\
-0.2926 \\
\end{array}$ & $\begin{array}{l}-0.1406 \\
-0.1544 \\
-0.1588 \\
\end{array}$ & $\begin{array}{l}-0.3498 \\
-0.3450 \\
-0.3509 \\
\end{array}$ & $\begin{array}{l}-0.3957 \\
-0.3920 \\
-0.3844 \\
\end{array}$ & $\begin{array}{l}0.50 \\
0.51 \\
0.51\end{array}$ & $\begin{array}{l}0.72 \\
0.70 \\
0.69\end{array}$ & $\begin{array}{l}0.45 \\
0.45 \\
0.45\end{array}$ & $\begin{array}{l}0.40 \\
0.41 \\
0.41\end{array}$ \\
\hline $\operatorname{cst} 07$ & $\begin{array}{l}\text { Meas. } \\
\text { Meas. bc } \\
\text { Target }\end{array}$ & $\begin{array}{l}-0.1103 \\
-0.1050 \\
-0.1299 \\
\end{array}$ & $\begin{array}{l}-0.0262 \\
-0.0422 \\
-0.0529 \\
\end{array}$ & $\begin{array}{l}-0.3164 \\
-0.3115 \\
-0.2849 \\
\end{array}$ & $\begin{array}{l}-0.2822 \\
-0.2785 \\
-0.2879 \\
\end{array}$ & $\begin{array}{l}0.78 \\
0.79 \\
0.74 \\
\end{array}$ & $\begin{array}{l}0.94 \\
0.91 \\
0.89 \\
\end{array}$ & $\begin{array}{l}0.48 \\
0.49 \\
0.52 \\
\end{array}$ & $\begin{array}{l}0.52 \\
0.53 \\
0.52 \\
\end{array}$ & $\begin{array}{l}-0.1345 \\
-0.1291 \\
-0.1541 \\
\end{array}$ & $\begin{array}{l}-0.0555 \\
-0.0714 \\
-0.0822 \\
\end{array}$ & $\begin{array}{l}-0.2991 \\
-0.2942 \\
-0.2675 \\
\end{array}$ & $\begin{array}{l}-0.2944 \\
-0.2907 \\
-0.3001 \\
\end{array}$ & $\begin{array}{l}0.73 \\
0.74 \\
0.70 \\
\end{array}$ & $\begin{array}{l}0.88 \\
0.85 \\
0.83 \\
\end{array}$ & $\begin{array}{l}0.50 \\
0.51 \\
0.54 \\
\end{array}$ & $\begin{array}{l}0.51 \\
0.51 \\
0.50 \\
\end{array}$ \\
\hline cst08 & $\begin{array}{l}\text { Meas. } \\
\text { Meas. bc } \\
\text { Target }\end{array}$ & $\begin{array}{r}-0.1332 \\
-0.1263 \\
-0.1307 \\
\end{array}$ & $\begin{array}{r}-0.0466 \\
-0.0603 \\
-0.0596 \\
\end{array}$ & $\begin{array}{l}-0.2891 \\
-0.2842 \\
-0.2682 \\
\end{array}$ & $\begin{array}{l}-0.3576 \\
-0.3539 \\
-0.3383 \\
\end{array}$ & $\begin{array}{l}0.74 \\
0.75 \\
0.74 \\
\end{array}$ & $\begin{array}{l}0.90 \\
0.87 \\
0.87 \\
\end{array}$ & $\begin{array}{l}0.51 \\
0.52 \\
0.54 \\
\end{array}$ & $\begin{array}{l}0.44 \\
0.44 \\
0.46 \\
\end{array}$ & $\begin{array}{r}-0.2603 \\
-0.2534 \\
-0.2578 \\
\end{array}$ & $\begin{array}{l}-0.1444 \\
-0.1581 \\
-0.1575 \\
\end{array}$ & $\begin{array}{l}-0.3324 \\
-0.3275 \\
-0.3114 \\
\end{array}$ & $\begin{array}{l}-0.3732 \\
-0.3694 \\
-0.3539 \\
\end{array}$ & $\begin{array}{l}0.55 \\
0.56 \\
0.55 \\
\end{array}$ & $\begin{array}{l}0.72 \\
0.69 \\
0.70 \\
\end{array}$ & $\begin{array}{l}0.47 \\
0.47 \\
0.49 \\
\end{array}$ & $\begin{array}{l}0.42 \\
0.43 \\
0.44 \\
\end{array}$ \\
\hline cst09 & $\begin{array}{c}\text { Meas. } \\
\text { Meas. bc } \\
\text { Target }\end{array}$ & $\begin{array}{r}-0.1669 \\
-0.1615 \\
-0.1800 \\
\end{array}$ & $\begin{array}{r}-0.0325 \\
-0.0484 \\
-0.0653 \\
\end{array}$ & $\begin{array}{r}-0.3179 \\
-0.3168 \\
-0.3307 \\
\end{array}$ & $\begin{array}{r}-0.3680 \\
-0.3666 \\
-0.3515 \\
\end{array}$ & $\begin{array}{l}0.68 \\
0.69 \\
0.66 \\
\end{array}$ & $\begin{array}{l}0.93 \\
0.89 \\
0.86 \\
\end{array}$ & $\begin{array}{l}0.48 \\
0.48 \\
0.47 \\
\end{array}$ & $\begin{array}{l}0.43 \\
0.43 \\
0.45 \\
\end{array}$ & $\begin{array}{r}-0.1978 \\
-0.1925 \\
-0.2109 \\
\end{array}$ & $\begin{array}{l}-0.0739 \\
-0.0898 \\
-0.1067 \\
\end{array}$ & $\begin{array}{r}-0.3114 \\
-0.3103 \\
-0.3242 \\
\end{array}$ & $\begin{array}{r}-0.3852 \\
-0.3838 \\
-0.3687 \\
\end{array}$ & $\begin{array}{l}0.63 \\
0.64 \\
0.62 \\
\end{array}$ & $\begin{array}{l}0.84 \\
0.81 \\
0.78 \\
\end{array}$ & $\begin{array}{l}0.49 \\
0.49 \\
0.47 \\
\end{array}$ & $\begin{array}{l}0.41 \\
0.41 \\
0.43 \\
\end{array}$ \\
\hline $\operatorname{cst} 10$ & $\begin{array}{l}\text { Meas. } \\
\text { Meas. bc } \\
\text { Target }\end{array}$ & $\begin{array}{l}-0.2593 \\
-0.2525 \\
-0.2610 \\
\end{array}$ & $\begin{array}{l}-0.0930 \\
-0.1068 \\
-0.1153 \\
\end{array}$ & $\begin{array}{l}-0.5113 \\
-0.5102 \\
-0.5041 \\
\end{array}$ & $\begin{array}{l}-0.4143 \\
-0.4129 \\
-0.3994 \\
\end{array}$ & $\begin{array}{l}0.55 \\
0.56 \\
0.55 \\
\end{array}$ & $\begin{array}{l}0.81 \\
0.78 \\
0.77 \\
\end{array}$ & $\begin{array}{l}0.31 \\
0.31 \\
0.31 \\
\end{array}$ & $\begin{array}{l}0.39 \\
0.39 \\
0.40 \\
\end{array}$ & $\begin{array}{l}-0.3840 \\
-0.3772 \\
-0.3856 \\
\end{array}$ & $\begin{array}{l}-0.1977 \\
-0.2115 \\
-0.2200 \\
\end{array}$ & $\begin{array}{l}-0.5378 \\
-0.5366 \\
-0.5306 \\
\end{array}$ & $\begin{array}{l}-0.4475 \\
-0.4461 \\
-0.4326 \\
\end{array}$ & $\begin{array}{l}0.41 \\
0.42 \\
0.41 \\
\end{array}$ & $\begin{array}{l}0.63 \\
0.61 \\
0.60 \\
\end{array}$ & $\begin{array}{l}0.29 \\
0.29 \\
0.29 \\
\end{array}$ & $\begin{array}{l}0.36 \\
0.36 \\
0.37 \\
\end{array}$ \\
\hline $\operatorname{cst} 11$ & $\begin{array}{l}\text { Meas. } \\
\text { Meas. bc } \\
\text { Target }\end{array}$ & $\begin{array}{r}-0.2634 \\
-0.2566 \\
-0.2710 \\
\end{array}$ & $\begin{array}{l}-0.0790 \\
-0.0928 \\
-0.1028 \\
\end{array}$ & $\begin{array}{l}-0.5885 \\
-0.5837 \\
-0.5846 \\
\end{array}$ & $\begin{array}{l}-0.4131 \\
-0.4093 \\
-0.4051 \\
\end{array}$ & $\begin{array}{l}0.55 \\
0.55 \\
0.54 \\
\end{array}$ & $\begin{array}{l}0.83 \\
0.81 \\
0.79 \\
\end{array}$ & $\begin{array}{l}0.26 \\
0.26 \\
0.26 \\
\end{array}$ & $\begin{array}{l}0.39 \\
0.39 \\
0.39 \\
\end{array}$ & $\begin{array}{l}-0.3479 \\
-0.3411 \\
-0.3555 \\
\end{array}$ & $\begin{array}{l}-0.1309 \\
-0.1446 \\
-0.1547 \\
\end{array}$ & $\begin{array}{l}-0.5578 \\
-0.5530 \\
-0.5539 \\
\end{array}$ & $\begin{array}{l}-0.4301 \\
-0.4264 \\
-0.4222 \\
\end{array}$ & $\begin{array}{l}0.45 \\
0.46 \\
0.44 \\
\end{array}$ & $\begin{array}{l}0.74 \\
0.72 \\
0.70 \\
\end{array}$ & $\begin{array}{l}0.28 \\
0.28 \\
0.28 \\
\end{array}$ & $\begin{array}{l}0.37 \\
0.37 \\
0.38 \\
\end{array}$ \\
\hline cst12 & $\begin{array}{c}\text { Meas. } \\
\text { Meas. bc } \\
\text { Target }\end{array}$ & $\begin{array}{l}-0.2283 \\
-0.2230 \\
-0.2343\end{array}$ & $\begin{array}{l}-0.0724 \\
-0.0884 \\
-0.0992\end{array}$ & $\begin{array}{l}-0.4942 \\
-0.4930 \\
-0.4979\end{array}$ & $\begin{array}{l}-0.3828 \\
-0.3814 \\
-0.3700\end{array}$ & $\begin{array}{l}0.59 \\
0.60 \\
0.58\end{array}$ & $\begin{array}{l}0.85 \\
0.82 \\
0.80\end{array}$ & $\begin{array}{l}0.32 \\
0.32 \\
0.32\end{array}$ & $\begin{array}{l}0.41 \\
0.42 \\
0.43\end{array}$ & $\begin{array}{l}-0.2198 \\
-0.2145 \\
-0.2259\end{array}$ & $\begin{array}{l}-0.0938 \\
-0.1098 \\
-0.1206\end{array}$ & $\begin{array}{l}-0.4369 \\
-0.4357 \\
-0.4406\end{array}$ & $\begin{array}{l}-0.3741 \\
-0.3727 \\
-0.3613\end{array}$ & $\begin{array}{l}0.60 \\
0.61 \\
0.59\end{array}$ & $\begin{array}{l}0.81 \\
0.78 \\
0.76\end{array}$ & $\begin{array}{l}0.37 \\
0.37 \\
0.36\end{array}$ & $\begin{array}{l}0.42 \\
0.42 \\
0.44\end{array}$ \\
\hline cst13 & $\begin{array}{l}\text { Meas. } \\
\text { Meas. bc } \\
\text { Target }\end{array}$ & $\begin{array}{l}-0.2630 \\
-0.2562 \\
-0.2697 \\
\end{array}$ & $\begin{array}{l}-0.0877 \\
-0.1014 \\
-0.1134 \\
\end{array}$ & $\begin{array}{l}-0.4364 \\
-0.4353 \\
-0.4231 \\
\end{array}$ & $\begin{array}{l}-0.3892 \\
-0.3878 \\
-0.3752 \\
\end{array}$ & $\begin{array}{l}0.55 \\
0.55 \\
0.54 \\
\end{array}$ & $\begin{array}{l}0.82 \\
0.79 \\
0.77 \\
\end{array}$ & $\begin{array}{l}0.37 \\
0.37 \\
0.38 \\
\end{array}$ & $\begin{array}{l}0.41 \\
0.41 \\
0.42 \\
\end{array}$ & $\begin{array}{l}-0.2676 \\
-0.2609 \\
-0.2744 \\
\end{array}$ & $\begin{array}{l}-0.1116 \\
-0.1254 \\
-0.1373 \\
\end{array}$ & $\begin{array}{l}-0.4193 \\
-0.4182 \\
-0.4060 \\
\end{array}$ & $\begin{array}{l}-0.3967 \\
-0.3953 \\
-0.3828 \\
\end{array}$ & $\begin{array}{l}0.54 \\
0.55 \\
0.53 \\
\end{array}$ & $\begin{array}{l}0.77 \\
0.75 \\
0.73 \\
\end{array}$ & $\begin{array}{l}0.38 \\
0.38 \\
0.39 \\
\end{array}$ & $\begin{array}{l}0.40 \\
0.40 \\
0.41 \\
\end{array}$ \\
\hline cst14 & $\begin{array}{l}\text { Meas. } \\
\text { Meas. bc } \\
\text { Target }\end{array}$ & $\begin{array}{l}-0.3533 \\
-0.3465 \\
-0.3544\end{array}$ & $\begin{array}{l}-0.1231 \\
-0.1369 \\
-0.1512\end{array}$ & $\begin{array}{l}-0.4673 \\
-0.4661 \\
-0.4639\end{array}$ & $\begin{array}{l}-0.3726 \\
-0.3712 \\
-0.3676\end{array}$ & $\begin{array}{l}0.44 \\
0.45 \\
0.44\end{array}$ & $\begin{array}{l}0.75 \\
0.73 \\
0.71\end{array}$ & $\begin{array}{l}0.34 \\
0.34 \\
0.34\end{array}$ & $\begin{array}{l}0.42 \\
0.43 \\
0.43\end{array}$ & $\begin{array}{l}-0.3759 \\
-0.3691 \\
-0.3770\end{array}$ & $\begin{array}{l}-0.1652 \\
-0.1790 \\
-0.1932\end{array}$ & $\begin{array}{l}-0.4560 \\
-0.4548 \\
-0.4526\end{array}$ & $\begin{array}{l}-0.3861 \\
-0.3847 \\
-0.3811\end{array}$ & $\begin{array}{l}0.42 \\
0.43 \\
0.42\end{array}$ & $\begin{array}{l}0.68 \\
0.66 \\
0.64\end{array}$ & $\begin{array}{l}0.35 \\
0.35 \\
0.35\end{array}$ & $\begin{array}{l}0.41 \\
0.41 \\
0.42\end{array}$ \\
\hline
\end{tabular}

2 Shaded rows in this table indicate glasses whose PCTs would not be considered valid due to less than 2 good results out of the 3 triplicates conducted for that glass. Missing values indicate that none of the three PCTs for the indicated glass were considered valid. 
WSRC-TR-2001-00125

Revision 0

Table 5: Normalized PCTs after Screening for Solution-Weight Problems (continued)

\begin{tabular}{|c|c|c|c|c|c|c|c|c|c|c|c|c|c|c|c|c|c|}
\hline \multirow[b]{2}{*}{$\begin{array}{l}\text { Glass } \\
\text { ID }\end{array}$} & \multirow{3}{*}{$\begin{array}{c}\text { Composition } \\
\text { Meas. }\end{array}$} & \multicolumn{8}{|c|}{ Quenched } & \multicolumn{8}{|c|}{ Centerline Cooled } \\
\hline & & $\begin{array}{c}\log N L \\
{[B(g / L)]}\end{array}$ & $\begin{array}{c}\log \mathrm{NL} \\
{[\mathrm{Si}(\mathrm{g} / \mathrm{L})]}\end{array}$ & $\begin{array}{c}\log \mathrm{NL} \\
{[\mathrm{Na}(\mathrm{g} / \mathrm{L})]}\end{array}$ & $\begin{array}{c}\log \mathrm{NL} \\
{[\mathrm{Li}(\mathrm{g} / \mathrm{L})]}\end{array}$ & $\begin{array}{c}\mathrm{NL} \\
\mathrm{B}(\mathrm{g} / \mathrm{L})\end{array}$ & $\begin{array}{c}\mathrm{NL} \\
\mathrm{Si}(\mathrm{g} / \mathrm{L})\end{array}$ & $\begin{array}{c}\mathrm{NL} \\
\mathrm{Na}(\mathrm{g} / \mathrm{L})\end{array}$ & $\begin{array}{c}\mathrm{NL} \\
\mathrm{Li}(\mathrm{g} / \mathrm{L})\end{array}$ & $\begin{array}{c}\log \mathrm{NL} \\
{[\mathrm{B}(\mathrm{g} / \mathrm{L})]}\end{array}$ & $\begin{array}{c}\log \mathrm{NL} \\
{[\mathrm{Si}(\mathrm{g} / \mathrm{L})]}\end{array}$ & $\begin{array}{c}\log \mathrm{NL} \\
\mathrm{Na}(\mathrm{g} / \mathrm{L})\end{array}$ & $\begin{array}{c}\log \mathrm{NL} \\
{[\mathrm{Li}(\mathrm{g} / \mathrm{L}) \mathrm{C}}\end{array}$ & $\begin{array}{c}\mathrm{NL} \\
\mathrm{B}(\mathrm{g} / \mathrm{L})\end{array}$ & $\begin{array}{c}\mathrm{NL} \\
\mathrm{Si}(\sigma / \mathrm{L})\end{array}$ & $\begin{array}{c}\mathrm{NL} \\
\mathrm{Na}(\mathrm{g} / \mathrm{L})\end{array}$ & $\begin{array}{c}\mathrm{NL} \\
\mathrm{Li}(\mathrm{g} / \mathrm{L})\end{array}$ \\
\hline \multirow{3}{*}{$\operatorname{cst} 15$} & & -0.2433 & -0.0849 & -0.4144 & -0.3717 & 0.57 & 0.82 & 0.39 & 0.42 & -0.2745 & -0.1296 & -0.3969 & -0.3921 & 0.53 & 0.74 & 0.40 & $\begin{array}{c}0.41 \\
0.41\end{array}$ \\
\hline & Meas. bc & -0.2365 & -0.0986 & -0.4133 & -0.3703 & 0.58 & 0.80 & 0.39 & 0.43 & -0.2678 & -0.1434 & -0.3957 & -0.3907 & 0.54 & 0.72 & 0.40 & 0.41 \\
\hline & Target & -0.2524 & -0.1129 & -0.4149 & -0.3598 & 0.56 & 0.77 & 0.38 & 0.44 & -0.2837 & -0.1576 & -0.3974 & -0.3802 & 0.52 & 0.70 & 0.40 & 0.42 \\
\hline \multirow{3}{*}{$\operatorname{cst} 16$} & Meas. & -0.3114 & -0.1495 & -0.3566 & -0.3772 & 0.49 & 0.71 & 0.44 & 0.42 & -0.3067 & -0.1652 & -0.3372 & -0.3600 & 0.49 & 0.68 & 0.46 & 0.44 \\
\hline & Meas. bc & -0.3045 & -0.1632 & -0.3554 & -0.3758 & 0.50 & 0.69 & 0.44 & 0.42 & -0.2998 & -0.1790 & -0.3360 & -0.3586 & 0.50 & 0.66 & 0.46 & 0.44 \\
\hline & Target & -0.3239 & -0.1700 & -0.3581 & -0.3687 & 0.47 & 0.68 & 0.44 & 0.43 & -0.3192 & -0.1858 & -0.3387 & -0.3516 & 0.48 & 0.65 & 0.46 & 0.45 \\
\hline \multirow{3}{*}{ cst17 } & Meas. & -0.2150 & -0.0957 & -0.2995 & -0.3540 & 0.61 & 0.80 & 0.50 & 0.44 & -0.2589 & -0.1461 & -0.3075 & -0.3634 & 0.55 & 0.71 & 0.49 & 0.43 \\
\hline & Meas. bc & -0.2096 & -0.1115 & -0.2946 & -0.3503 & 0.62 & 0.77 & 0.51 & 0.45 & -0.2535 & -0.1620 & -0.3027 & -0.3597 & 0.56 & 0.69 & 0.50 & 0.44 \\
\hline & Target & -0.2193 & -0.1128 & -0.2979 & -0.3428 & 0.60 & 0.77 & 0.50 & 0.45 & -0.2632 & -0.1632 & -0.3059 & -0.3523 & 0.55 & 0.69 & 0.49 & 0.44 \\
\hline
\end{tabular}

Also, note that the EA PCT response from Tables 4 and 5 is lower than that typically seen for this standard glass [8]. However, the ARM results are at the upper end of their historical values as reported in [8].

As seen in Tables 4 and 5, the durabilities for the CST glasses are much better than those of EA. (This is indicated for each glass by its normalized leachate being much smaller than that of EA.). Figures 13 provide an opportunity for a closer look at just the valid PCTs using measured, bias-corrected, and targeted compositions. Each of these figures is a plot of the DWPF model that relates the logarithm of the normalized PCT (in this case for B) to a linear function of a free energy of hydration term ( $\Delta \mathrm{G}_{\mathrm{p}}$, $\mathrm{kcal} / 100 \mathrm{~g}$ glass) derived from the glass (measured, bias-corrected, or targeted) compositions [8]. Prediction limits (at 95\% confidence) for individual PCT results are also plotted around this linear fit. The PCT results for EA (shown as a diamond at $\Delta \mathrm{G}_{\mathrm{p}} \sim-15.5$ ), ARM (shown as a diamond at $\Delta \mathrm{G}_{\mathrm{p}}$ slightly above -10), and the CST glasses (quenched shown as small, closed squares and centerline cooled shown as open circles) are presented on this plot. Note that the CST results reveal acceptable PCTs and that the PCT predictions by the current DWPF durability model for boron mimic the behavior seen in [3]. Specifically, at the more positive $\Delta \mathrm{G}_{\mathrm{p}}$ values for the CST glasses, the model tends to predict durabilities that are lower (more durable) than measured. Exhibit A.6 in Appendix A repeats the plots of Figures 1-3, provides the same plots for $\mathrm{Li}, \mathrm{Na}$, and $\mathrm{Si}$ for the valid PCTs and provides similar plots for $\mathrm{B}, \mathrm{Li}, \mathrm{Na}$, and $\mathrm{Si}$ for the unscreened and screened PCTs. The behavior seen in these plots for $\mathrm{B}, \mathrm{Li}, \mathrm{Na}$, and $\mathrm{Si}$ is similar to that demonstrated by the B results in Figures 1-3: acceptable but less than ideally predictable durabilities. For these plots, the Na behavior reveals more of a slope in the PCT responses than the other elements.

Figure 1.

$\log \mathrm{NL}[\mathrm{B}(\mathrm{g} / \mathrm{L})]$ By del Gp. (Valid PCTs \& Measured Compositions)

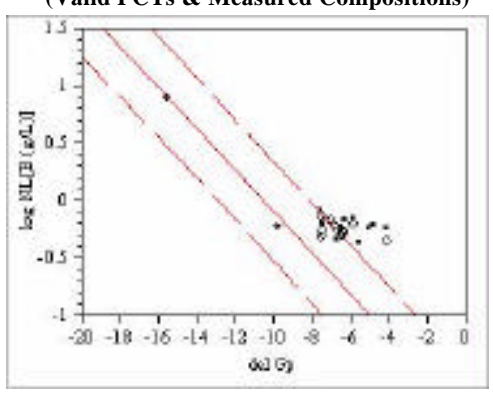

Figure 2. $\log \mathrm{NL}[\mathrm{B}(\mathrm{g} / \mathrm{L})]$ By del Gp

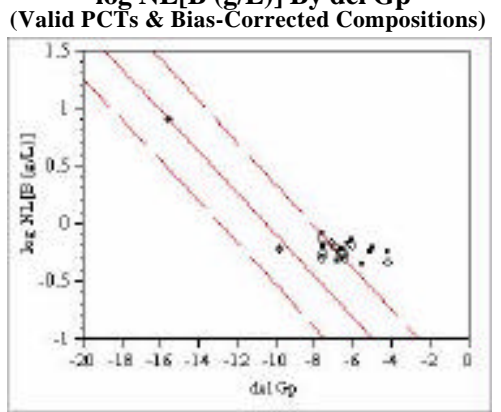

Figure 3.

$\log \mathrm{NL}[\mathrm{B}(\mathrm{g} / \mathrm{L})]$ By del Gp (Valid PCTs \& Targeted Compositions)

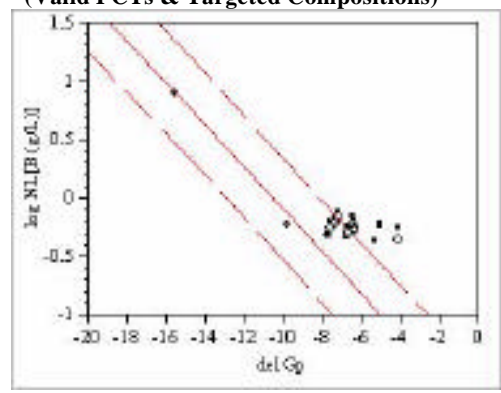

A look at the reproducibility of the PCT results between this and the FY99 study is provided in Table 6. This table provides the normalized PCT results (in grams/liter) from [3] for the FY99 glasses of interest and from Tables 4 and 5 for the quenched versions of those glasses repeated in this study. Recall that only quenched glasses were studied in the FY99 work. Good reproducibility between the two studies is demonstrated by the results presented in Table 6. 
WSRC-TR-2001-00125

Revision 0

\section{Table 6: Normalized PCTs for Glasses Common to the FY01 and FY99 Studies}

\begin{tabular}{|c|c|c|c|c|c|c|c|c|c|c|c|}
\hline $\begin{array}{l}\text { Study \& } \\
\text { Glass ID }\end{array}$ & $\begin{array}{c}\text { Composition } \\
\text { Represented By }\end{array}$ & $\begin{array}{c}\mathrm{NL} \\
\mathrm{B}(\mathrm{g} / \mathrm{L})\end{array}$ & $\begin{array}{c}\mathrm{NL} \\
\mathrm{Li}(\mathrm{g} / \mathrm{L})\end{array}$ & $\begin{array}{c}\mathrm{NL} \\
\mathrm{Na}(\mathrm{g} / \mathrm{L})\end{array}$ & $\begin{array}{c}\mathrm{NL} \\
\mathrm{Si}(\mathrm{g} / \mathrm{L}) \\
\end{array}$ & $\begin{array}{c}\text { Glass } \\
\text { ID }\end{array}$ & Composition & $\begin{array}{c}\mathrm{NL} \\
\mathrm{B}(\mathrm{g} / \mathrm{L})\end{array}$ & $\begin{array}{c}\mathrm{NL} \\
\mathrm{Li}(\mathrm{g} / \mathrm{L}) \\
\end{array}$ & $\begin{array}{c}\mathrm{NL} \\
\mathrm{Na}(\mathrm{g} / \mathrm{L}) \\
\end{array}$ & $\begin{array}{c}\mathrm{NL} \\
\mathrm{Si}(\mathrm{g} / \mathrm{L}) \\
\end{array}$ \\
\hline \multirow{3}{*}{ FY01 cst01 } & Measured & 0.64 & 0.84 & 0.44 & 0.41 & \multirow{3}{*}{ FY01 cst02 } & Measured & 0.42 & 0.77 & 0.20 & 0.38 \\
\hline & Measured bias-cor. & 0.65 & 0.81 & 0.45 & 0.41 & & Measured bias-cor. & 0.42 & 0.74 & 0.20 & 0.39 \\
\hline & Targeted & 0.62 & 0.78 & 0.45 & 0.42 & & Targeted & 0.41 & 0.73 & 0.21 & 0.39 \\
\hline \multirow{3}{*}{ FY99 cst $12 \mathrm{c}$} & Measured & 0.72 & 0.86 & 0.51 & 0.44 & \multirow{3}{*}{ FY99 cst 26} & Measured & 0.41 & 0.70 & 0.25 & 0.38 \\
\hline & Measured bias-cor. & 0.72 & 0.89 & 0.53 & 0.44 & & Measured bias-cor. & 0.41 & 0.73 & 0.25 & 0.37 \\
\hline & Targeted & 0.68 & 0.86 & 0.52 & 0.43 & & Targeted & 0.42 & 0.71 & 0.25 & 0.37 \\
\hline
\end{tabular}

\section{Quenched versus Centerline-Cooled PCTs}

A primary objective of this study was the investigation of potential cooling effects on the durability of the CST glasses. Figures 1-3 and Exhibit A.6 provide a first look at differences in PCT response due to the cooling regime for the glasses. From those plots, there does not appear to be a lack of PCT predictability or acceptability due to cooling regime. A more rigorous comparison of the PCTs from quenched versus centerline-cooled glasses is provided in this section.

Exhibit A.7 in Appendix A provides paired comparisons (made using JMP® Version $4 .^{3}$ ) of the average common logarithm of the leachate concentrations in parts per million. The data that are averaged are provided in Table A.1 of Appendix A. The exhibit investigates the unscreened, screened, and valid PCTs in turn. For boron (regardless of the PCT group), there is a statistically significant difference (at the 5\% significance level) between the quenched and centerline-cooled results with the boron PCT releases from the quenched glasses being slightly higher than those from the centerlinecooled glasses. In log space, the difference (on average) is less than 0.03 , which is of no real, practical concern. The lithium results mirror those for boron, with the average difference being less than 0.05 in $\log$ space and statistically significant at the $5 \%$ level.

The results for silicon indicate no statistically significant (at the 5\% level) difference in the PCTs due to the heat treatment of the glasses. The mean difference is less than 0.01 in log space for these measurements.

The results for sodium indicate a statistically significant difference (at the 5\% level) in the PCTs for only the valid PCTs. In log space, the quenched PCTs are slightly ( 0.02$)$ smaller than the centerlinecooled PCTs. Thus, the behavior of the Na response is somewhat different from the behaviors demonstrated for the other elements.

Another look at quenched versus centerline-cooled PCTs for boron is provided in Figures 4-7 for the valid PCTs. These plots provide quenched versus centerline cooled scatter plots with the $\mathrm{x}$ and $\mathrm{y}$ axes drawn to the same scale. If the PCT is not affected by cooling regime, the quenched and centerlinecooled pairs should fall along the diagonal line for each of these plots.

3 JMP $®$ Version 4.0 is a commercial software product of SAS Institute, Inc. in Cary, NC. This product was used to support the statistical analyses presented in this report. See JMP® Statistical Discovery Software: Statistics and Graphics Guide (2000) published by SAS Institute for details. 
WSRC-TR-2001-00125

Revision 0

Figure 4.

Special Linear Fit of $\log$ NL[B (g/L)] clc By $\log N L[B(g / L)]$ quenched

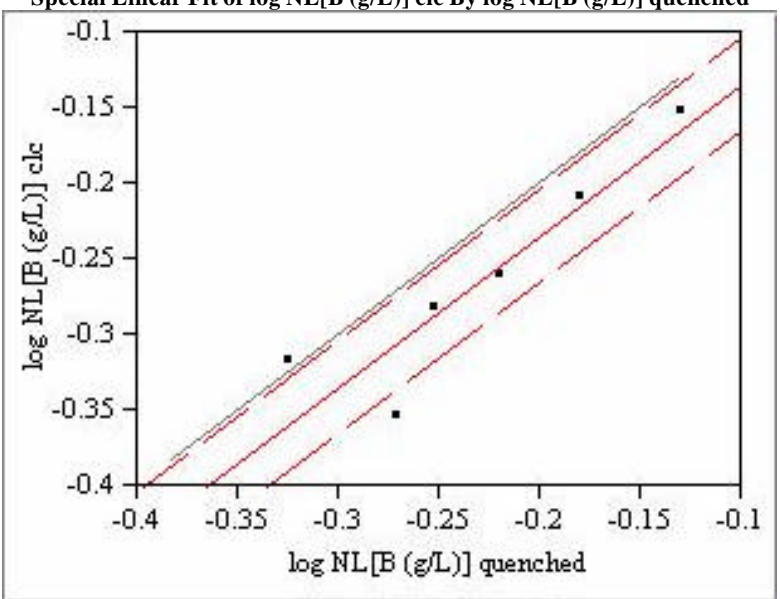

Figure 5.

Special Linear Fit of $\log \mathrm{NL}[\mathrm{Li}(\mathrm{g} / \mathrm{L})]$ clc By $\log \mathrm{NL}[\mathrm{Li}(\mathrm{g} / \mathrm{L})]$ quenched

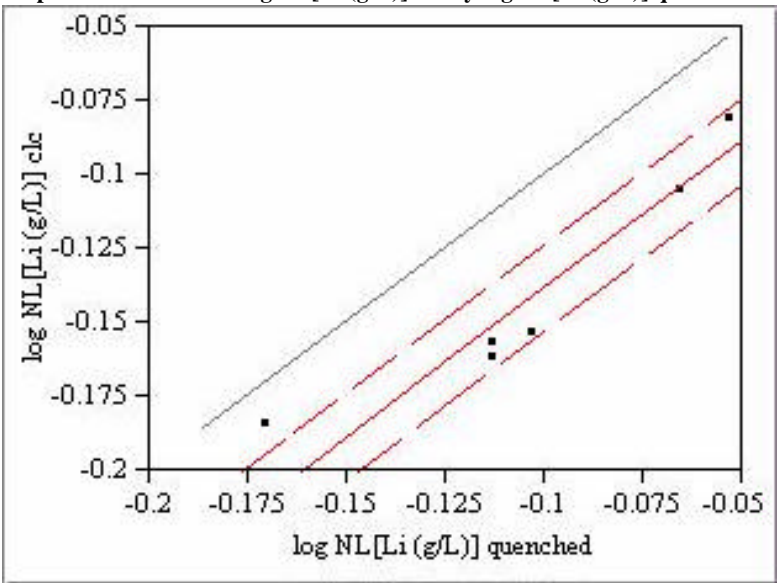

Figure 6.

Special Linear Fit of $\log \mathrm{NL}[\mathrm{Na}(\mathrm{g} / \mathrm{L})]$ clc By $\log \mathrm{NL}[\mathrm{Na}(\mathrm{g} / \mathrm{L})]$ quenched

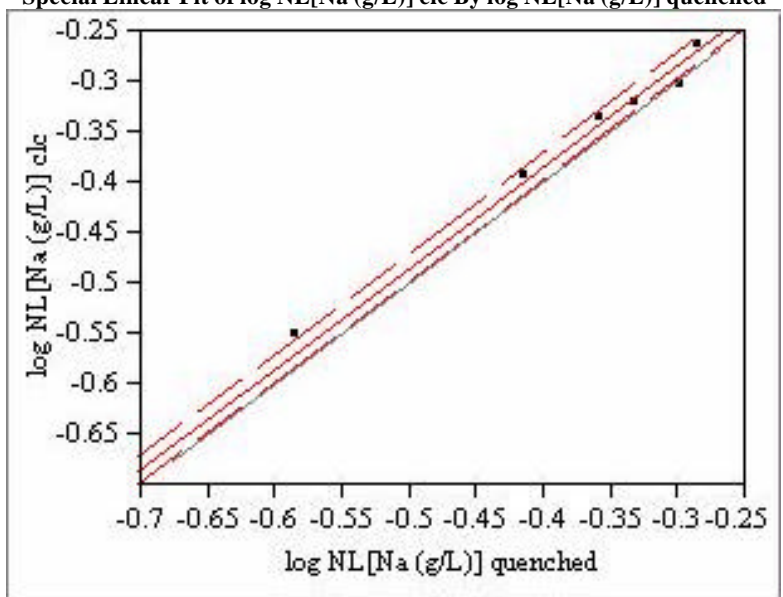

Figure 7.

Special Linear Fit of $\log \mathrm{NL}[\mathrm{Si}(\mathrm{g} / \mathrm{L})]$ clc By NL[Si (g/L)] quenched

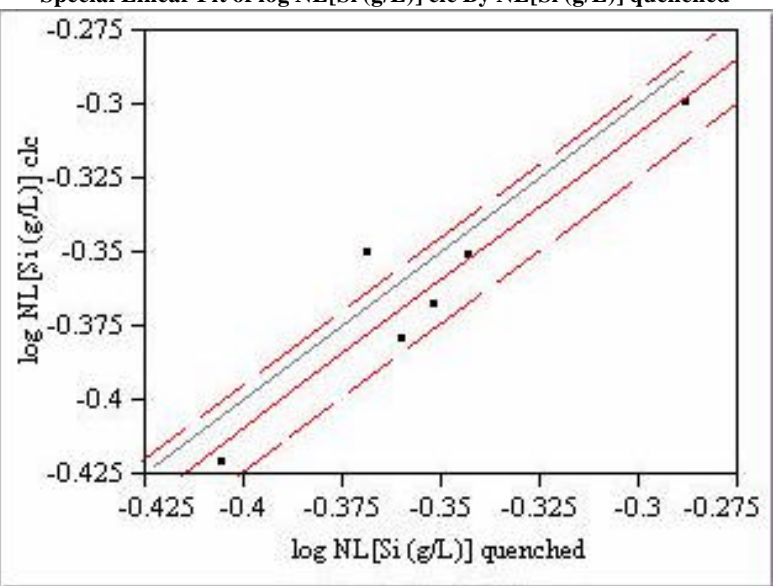

These results indicate no impact (of practical importance) between the two cooling regimes (quenched and centerline cooled) for the CST glasses of this study.

\section{Phase Separation}

The formation of separate amorphous phases in glass is referred to as amorphous phase separation or inhomogeneity. Crystal formation, as determined by liquidus temperature measurements on the other hand, may indicate a "separation of phases," but reflects crystalline particles within the glass matrix. Amorphous phase separation is to be avoided since the models currently used to predict durability do not apply for glasses predicted to be phase separated. The limit for the homogeneity constraint in the PCCS is nominally (for the Property Acceptance Region, PAR) a value of 211 [4]. For the measurement acceptance region (MAR), the value will be even higher. In order for a glass to pass this constraint, the calculated value from chemical composition of the glass must be greater than the MAR value. The predicted values for the CST glasses are given in Table 7. All of the targeted compositions except for cst08 are below the PAR and are, thus, predicted to be phase separated. For the measured compositions, only cst06, cst08, and cst12 pass the PAR. For the bias-corrected compositions, only cst08 and cst12 fall within the PAR. 
WSRC-TR-2001-00125

Revision 0

Table 7: Homogeneity Property Predictions

\begin{tabular}{|c|c|c|c|}
\hline \multirow[b]{2}{*}{ Glass ID } & \multicolumn{3}{|c|}{$\begin{array}{c}\text { Homogeneity Property Prediction Based on } \\
\text { - Acceptability Requires a Value > } 211 \text { - } \\
\text { (Shaded Values are Acceptable) }\end{array}$} \\
\hline & $\begin{array}{c}\text { Target } \\
\text { Composition }\end{array}$ & $\begin{array}{c}\text { Measured } \\
\text { Composition }\end{array}$ & $\begin{array}{c}\text { Bias-Corrected } \\
\text { Composition }\end{array}$ \\
\hline cst01 & 197.8 & 202.5 & 200.6 \\
\hline $\operatorname{cst02}$ & 204.9 & 206.6 & 205.8 \\
\hline cst03 & 186.2 & 187.8 & 186.2 \\
\hline cst04 & 205.5 & 205.1 & 205.1 \\
\hline $\operatorname{cst05}$ & 207.3 & 210.2 & 209.8 \\
\hline cst06 & 209.7 & 211.8 & 209.6 \\
\hline cst07 & 203.3 & 202.2 & 200.4 \\
\hline cst08 & 214.6 & 216.6 & 214.2 \\
\hline $\operatorname{cst} 09$ & 197.5 & 197.8 & 197.6 \\
\hline cst10 & 208.9 & 207.9 & 207.7 \\
\hline cst11 & 209.8 & 207.2 & 205.3 \\
\hline cst12 & 223.3 & 219.5 & 219.6 \\
\hline cst13 & 206.5 & 207.6 & 207.4 \\
\hline cst14 & 204.9 & 203.2 & 203.3 \\
\hline cst15 & 206.5 & 206.8 & 206.6 \\
\hline cst16 & 204.9 & 202.4 & 202.6 \\
\hline cst17 & 206.5 & 208.7 & 207.1 \\
\hline
\end{tabular}

The homogeneity constraint was developed for a glass compositional region that does not include all of the components introduced by CST. Therefore, the predictability of phase separation by this model may be questionable. A significant search for amorphous phase separation in these glasses is beyond the scope of work for this task, except when routine SEM analysis is performed.

\section{XRD and SEM Analysis of the Glasses}

None of the quenched glasses exhibited any visually apparent crystalline phases. However, several of the centerline-cooled glasses did have separate phases as observed visually. These glasses were submitted to ADS for X-Ray Diffraction (XRD) and Scanning Electron Microscopy (SEM) analyses. This section presents the results of these analyses.

XRD results were obtained for the following clc glasses:

$\begin{array}{ll}\operatorname{cst} 01 & \text { Amorphous } \\ \text { cst05 } & \text { Amorphous } \\ \text { cst06 } & \text { Hematite and Trevorite and an unidentified phase } \\ \text { cst08 } & \text { Hematite and Trevorite } \\ \text { cst11 } & \text { Hematite plus a small amount of an unidentified phase } \\ \text { cst14 } & \text { Amorphous }\end{array}$

Glasses cst01, cst05, and cst14 were amorphous showing no crystalline phases to the sensitivity of XRD ( 0.5 to $1 \mathrm{wt} \%$ crystals).

Glasses cst06 and cst08 contained Purex sludge at $30 \mathrm{wt} \%$ oxide and cst 11 contained 28 wt $\%$ oxide of the Blend sludge. Therefore, at the higher sludge loadings, it appears that hematite can phase separate. Due to the limited scope for this part of the task, no XRD data was obtained for the other high-sludgeloaded glasses produced under quenched or centerline-cooled conditions.

SEM results were obtained for phases that appear on the surface of the glass. A variety of crystals were observed on the glass surfaces. For cst01, a glass with maximum titanium ( 5 wt $\%$ oxide), spinels enriched with titanium were evident along with a few rod-like structures of titanium. Glass cst05, with lesser titanium ( $3 \mathrm{wt} \%$ oxide), showed mainly trevorite with some crystals enriched with titanium. 
Glass cst06, with 4 wt $\%$ titanium oxide and $30 \mathrm{wt} \%$ oxide Purex sludge, exhibited crystals of spinel enriched with titanium, a phase rich in $\mathrm{Mn}$ and $\mathrm{Fe}$, and a rod-like phase rich in $\mathrm{Fe}$, $\mathrm{Ti}$, and $\mathrm{Si}$ (the $\mathrm{Si}$ could result from the matrix). The unidentified phase detected by XRD could correspond to one of the phases observed by SEM.

Glass cst11 has the maximum titanium ( $5 \mathrm{wt} \%$ as oxide) concentration in the glass and $28 \%$ blend sludge with high $\mathrm{Fe}$ concentration. Surface crystals of spinel enriched in titanium were observed. Both rod-like and spots of titanium phases were also observed by SEM. Finally a phase rich in Mn and $\mathrm{Fe}$ and similar in signature to the unidentified phase seen in cst06 was observed by SEM. The unidentified phase detected by XRD has similarities, but is evidently not identical, to the unidentified phase in cst06.

For glass cst12, a glass low in titanium ( $2 \mathrm{wt} \%$ as oxide) but high in sludge ( $30 \mathrm{wt} \%$ Blend), the majority of crystals were spinel with a smaller amount of crystals containing the high $\mathrm{Mn}$ and $\mathrm{Fe}$ phase. One spot was observed to contain higher concentrations of uranium.

For glass cst16, which contains $\mathrm{HM}$ sludge, crystals containing $\mathrm{Fe}, \mathrm{Mn}, \mathrm{Ti}, \mathrm{Ni}, \mathrm{Si}$ and $\mathrm{O}$ were observed. Spinel crystals were also detected.

It may also be worth noting that the presence of these crystalline phases had no apparent impact on the durabilities of these glasses.

\section{CONCLUSiOnS}

This study was conducted to determine the effect, if any, on the PCT responses of glasses cooled at different rates. The glasses contained CST and MST (plus a simulated sludge representing Purex, Blend, or HM) in amounts consistent with coupled operations using the CST option for salt disposition. Two bounding cooling profiles were used in this study: rapidly quenched and canister centerline cooling profiles. Glasses were selected based on a number of criteria, but mainly to challenge the regions where amorphous phase separation might be expected. Currently, DWPF utilizes a homogeneity constraint to preclude glass regions that could lead to phase separation. However, this constraint was not developed for a compositional region that includes the levels of CST (3-9 wt\%) and MST (2.5 wt $\%$ ) introduced by this study. Due to this uncertainty, the CST glasses that were selected for study covered a wide range of values for the homogeneity constraint. Also, it was important to ensure that deleterious phase separation does not occur for either cooling profile. In this case, deleterious phase separation is defined as the formation of amorphous phase separation (or glass-inglass) that significantly alters the glass durability as measured by the PCT response.

The results revealed that there was no practical difference between the PCT responses for glasses subjected to the two cooling profiles. In fact, although to a small extent, the boron PCT responses for the centerline-cooled glasses were more durable than the rapidly quenched glasses. These results reveal that no deleterious phase separation occurred under either cooling regime.

X-ray diffraction (XRD) and scanning electron microscopy (SEM) were used to evaluate phases observed in the centerline cooled glasses. XRD analysis, which detects crystals throughout the bulk of the glass, revealed that trevorite and hematite were present in some of the glasses.

SEM analysis of the surface crystals revealed a variety of different species including titanium rods and spots, trevorite, and trevorite enriched with titanium (appears to correlate with the amount of titanium in the glass), and a phase rich in $\mathrm{Mn}$ and Fe. One glass, cst12, contained a crystal high in uranium content.

The durabilities, as measured by the PCT response of boron, for the CST glasses ranged from 0.41 to $0.80 \mathrm{~g} / \mathrm{L}$. These values are significantly lower than the PCT values for glasses containing PHA (as 
reported in WSRC-TR-2001-00123) and for EA. The PCT results are highly clustered consistent with previous work. The measured PCT values fall above the upper $95 \%$ prediction interval of the DWPF model used to predict durability, again, consistent with previous results. The very good durability of the CST containing glasses implies that durability may not be the limiting factor for waste loading in this option.

\section{REFERENCES}

[1] “Technical Task: Request: Canister Centerline Cooling for CST Glass," HLW-SDT-TTR2000-00023, December 2000.

[2] Harbour, J. R. and T. B. Edwards, "Technical Task and QA Plan: Impact of Cooling Rates on the Durabilities of CST and PHA Glasses," WSRC-RP-2000-00996, December 2000.

[3] Edwards, T. B., J. R. Harbour, and R. J. Workman, "Summary of Property Measurements from CST Glass Study (U),” WSRC-TR-99-00384, Revision 0, October 4, 1999.

[4] Brown, K. G. and R. L. Postles, "SME Acceptability Determination for DWPF Process Control (U),”WSRC-TR-95-0364, Revision 3, February 21, 1996.

[5] ASTM C1285-97, "Standard Test Methods for Determining Chemical Durability of Nuclear Waste Glasses: The Product Consistency Test (PCT)," 1997.

[6] Brown, K. G. and T. B. Edwards, "Definition of the DWPF Homogeneity Constraint," WSRC-TR-95-0060, January 31, 1995.

[7] Harbour, J. R. and T. B. Edwards, “Analytical Study Plan: Impact of Cooling Rates on the Durabilities of CST and PHA Glasses,” WSRC-RP-2001-00080, Revision 0, January 3, 2001.

[8] Jantzen, C. M., J. B. Pickett, K. G. Brown, T. B. Edwards, and D. C. Beam, "Process/Product Models for the Defense Waste Processing Facility (DWPF): Part I. Predicting Glass Durability from Composition Using a Thermodynamic Hydration Energy Reaction Model (THERMO) (U)," WSRC-TR-93-672, Rev. 1, September 28, 1995. 
WSRC-TR-2001-00125

Revision 0

This page intentionally left blank. 
WSRC-TR-2001-00125

Revision 0

\section{APPENDIX A.}

\section{Supplemental Tables and Exhibits}


WSRC-TR-2001-00125

Revision 0

This page intentionally left blank. 
WSRC-TR-2001-00125

Revision 0

Table A.1: Composition of PCT Leachate Solutions

\begin{tabular}{|c|c|c|c|c|c|c|c|c|c|c|c|c|c|c|c|}
\hline & & & & \multicolumn{4}{|c|}{ Concentrations in ppm } & \multicolumn{4}{|c|}{ Concentrations in ppm } & \multirow{2}{*}{\multicolumn{4}{|c|}{ Common Logarithm of ppm Concentrations }} \\
\hline Glass & Lab & & & & (as re & orted) & & (af & correcti & for diluti & & & & & \\
\hline ID & ID & Blk & Seq & $\mathbf{B}$ & $\mathbf{L i}$ & $\mathbf{N a}$ & $\mathbf{S i}$ & $\mathbf{B}$ & $\mathbf{L i}$ & $\mathbf{N a}$ & $\mathbf{S i}$ & $\log [\mathbf{B}]$ & $\log [\mathbf{L i}]$ & $\log [\mathrm{Na}]$ & $\log [\mathrm{Si}]$ \\
\hline std & std-b1-1 & 1 & 1 & 19.7 & 9.38 & 81.4 & 49.8 & 19.700 & 9.380 & 81.400 & 49.800 & 1.2945 & 0.9722 & 1.9106 & 1.6972 \\
\hline $\operatorname{cst} 01$ & $\mathrm{cp} 056$ & 1 & 2 & 8.53 & 8.93 & 18.7 & 60.9 & 14.217 & 14.884 & 31.167 & 101.502 & 1.1528 & 1.1727 & 1.4937 & 2.0065 \\
\hline cst08 & $\mathrm{cp} 022$ & 1 & 3 & 9.31 & 9.52 & 21.7 & 62.2 & 15.517 & 15.867 & 36.167 & 103.669 & 1.1908 & 1.2005 & 1.5583 & 2.0156 \\
\hline cst $05 \mathrm{clc}$ & cp037 & 1 & 4 & 8.94 & 9.11 & 22.4 & 68.1 & 14.900 & 15.184 & 37.334 & 113.502 & 1.1732 & 1.1814 & 1.5721 & 2.0550 \\
\hline $\mathrm{cst} 17 \mathrm{clc}$ & cp067 & 1 & 5 & 6.66 & 7.92 & 23.4 & 64.8 & 11.100 & 13.200 & 39.001 & 108.002 & 1.0453 & 1.1206 & 1.5911 & 2.0334 \\
\hline $\operatorname{cst} 11$ & cp088 & 1 & 6 & 6.55 & 8.42 & 7.88 & 53.9 & 10.917 & 14.034 & 13.134 & 89.835 & 1.0381 & 1.1472 & 1.1184 & 1.9534 \\
\hline cst08clc & cp104 & 1 & 7 & 7.09 & 7.68 & 19.3 & 58.9 & 11.817 & 12.800 & 32.167 & 98.169 & 1.0725 & 1.1072 & 1.5074 & 1.9920 \\
\hline $\operatorname{cst} 03$ & cp110 & 1 & 8 & 8.03 & 9.32 & 20 & 64 & 13.384 & 15.534 & 33.334 & 106.669 & 1.1266 & 1.1913 & 1.5229 & 2.0280 \\
\hline cst05 & cp085 & 1 & 9 & 9.33 & 10.1 & 22.5 & 68.4 & 15.550 & 16.834 & 37.501 & 114.002 & 1.1917 & 1.2262 & 1.5740 & 2.0569 \\
\hline cst10 & cp070 & 1 & 10 & 6.72 & 8.27 & 10.3 & 55.2 & 11.200 & 13.784 & 17.167 & 92.002 & 1.0492 & 1.1394 & 1.2347 & 1.9638 \\
\hline std & std-b1-2 & 1 & 11 & 19.4 & 9.46 & 83.1 & 49.2 & 19.400 & 9.460 & 83.100 & 49.200 & 1.2878 & 0.9759 & 1.9196 & 1.6920 \\
\hline blank & $\mathrm{cp} 046$ & 1 & 12 & $<0.150$ & $<0.040$ & $<0.100$ & $<0.790$ & 0.125 & 0.033 & 0.083 & 0.658 & -0.9031 & -1.4771 & -1.0792 & -0.1816 \\
\hline cst01clc & cp108 & 1 & 13 & 7.46 & 7.86 & 17.4 & 59.9 & 12.434 & 13.100 & 29.001 & 99.835 & 1.0946 & 1.1173 & 1.4624 & 1.9993 \\
\hline cst16 & cp064 & 1 & 14 & 6.63 & 8.2 & 23.1 & 64.2 & 11.050 & 13.667 & 38.501 & 107.002 & 1.0434 & 1.1357 & 1.5855 & 2.0294 \\
\hline $\mathrm{cst} 03 \mathrm{clc}$ & cp069 & 1 & 15 & 8.14 & 8.71 & 18.1 & 66.1 & 13.567 & 14.517 & 30.167 & 110.169 & 1.1325 & 1.1619 & 1.4795 & 2.0421 \\
\hline cst11clc & cp059 & 1 & 16 & 5.58 & 7.48 & 8.58 & 53.2 & 9.300 & 12.467 & 14.300 & 88.668 & 0.9685 & 1.0958 & 1.1553 & 1.9478 \\
\hline cst12 & cp082 & 1 & 17 & 7.8 & 9.19 & 9.87 & 61.4 & 13.000 & 15.317 & 16.450 & 102.335 & 1.1140 & 1.1852 & 1.2162 & 2.0100 \\
\hline $\operatorname{cst} 17$ & cp045 & 1 & 18 & 8.3 & 9.17 & 23.2 & 64.1 & 13.834 & 15.284 & 38.667 & 106.836 & 1.1409 & 1.1842 & 1.5873 & 2.0287 \\
\hline cst12clc & cp055 & 1 & 19 & 6.59 & 7.43 & 10.2 & 54.2 & 10.984 & 12.384 & 17.000 & 90.335 & 1.0407 & 1.0928 & 1.2305 & 1.9559 \\
\hline cst $16 \mathrm{clc}$ & cp052 & 1 & 20 & 6.49 & 7.63 & 22.8 & 63.4 & 10.817 & 12.717 & 38.001 & 105.669 & 1.0341 & 1.1044 & 1.5798 & 2.0239 \\
\hline cst10clc & cp044 & 1 & 21 & 5.56 & 7.05 & 10.3 & 53.9 & 9.267 & 11.750 & 17.167 & 89.835 & 0.9669 & 1.0700 & 1.2347 & 1.9534 \\
\hline std & std-b1-3 & 1 & 22 & 19.7 & 9.53 & 83.3 & 50.1 & 19.700 & 9.530 & 83.300 & 50.100 & 1.2945 & 0.9791 & 1.9206 & 1.6998 \\
\hline std & std-b2-1 & 2 & 1 & 19.3 & 9.4 & 78.4 & 49.8 & 19.300 & 9.400 & 78.400 & 49.800 & 1.2856 & 0.9731 & 1.8943 & 1.6972 \\
\hline cst03 & ср020 & 2 & 2 & 7.48 & 8.72 & 15.6 & 59 & 12.467 & 14.534 & 26.001 & 98.335 & 1.0958 & 1.1624 & 1.4150 & 1.9927 \\
\hline $\mathrm{cst} 12 \mathrm{clc}$ & cp097 & 2 & 3 & 7.19 & 8.28 & 10.9 & 59.1 & 11.984 & 13.800 & 18.167 & 98.502 & 1.0786 & 1.1399 & 1.2593 & 1.9934 \\
\hline cst11clc & cp054 & 2 & 4 & 5.25 & 7.29 & 8.86 & 50.7 & 8.750 & 12.150 & 14.767 & 84.502 & 0.9420 & 1.0846 & 1.1693 & 1.9269 \\
\hline $\operatorname{cst} 11$ & cp084 & 2 & 5 & 6.3 & 8.24 & 8.06 & 51.9 & 10.500 & 13.734 & 13.434 & 86.502 & 1.0212 & 1.1378 & 1.1282 & 1.9370 \\
\hline $\operatorname{cst} 01$ & cp098 & 2 & 6 & 7.89 & 8.37 & 17.3 & 56.1 & 13.150 & 13.950 & 28.834 & 93.502 & 1.1189 & 1.1446 & 1.4599 & 1.9708 \\
\hline cst17 & cp003 & 2 & 7 & 8 & 9 & 22.2 & 62.9 & 13.334 & 15.000 & 37.001 & 104.835 & 1.1249 & 1.1761 & 1.5682 & 2.0205 \\
\hline cst10clc & cp053 & 2 & 8 & 5.26 & 6.65 & 9.97 & 51.6 & 8.767 & 11.084 & 16.617 & 86.002 & 0.9428 & 1.0447 & 1.2206 & 1.9345 \\
\hline cst $17 \mathrm{clc}$ & $\mathrm{cp} 015$ & 2 & 9 & 7.75 & 8.07 & 21.2 & 61.6 & 12.917 & 13.450 & 35.334 & 102.669 & 1.1112 & 1.1287 & 1.5482 & 2.0114 \\
\hline cst01clc & cp030 & 2 & 10 & & & & & & & & & & & & \\
\hline std & std-b2-2 & 2 & 11 & 19.1 & 9.34 & 78 & 49.5 & 19.100 & 9.340 & 78.000 & 49.500 & 1.2810 & 0.9703 & 1.8921 & 1.6946 \\
\hline cst16clc & $\mathrm{cp} 065$ & 2 & 12 & 6.81 & 8 & 23.4 & 66 & 11.350 & 13.334 & 39.001 & 110.002 & 1.0550 & 1.1249 & 1.5911 & 2.0414 \\
\hline cst $08 \mathrm{clc}$ & cp016 & 2 & 13 & 7.03 & 7.68 & 18.9 & 59 & 11.717 & 12.800 & 31.501 & 98.335 & 1.0688 & 1.1072 & 1.4983 & 1.9927 \\
\hline $\mathrm{cst} 03 \mathrm{clc}$ & cp048 & 2 & 14 & 7.73 & 8.61 & 17.8 & 64.2 & 12.884 & 14.350 & 29.667 & 107.002 & 1.1100 & 1.1569 & 1.4723 & 2.0294 \\
\hline cst05 & cp109 & 2 & 15 & 8.98 & 9.68 & 20.9 & 66.9 & 14.967 & 16.134 & 34.834 & 111.502 & 1.1751 & 1.2077 & 1.5420 & 2.0473 \\
\hline cst 10 & cp029 & 2 & 16 & 6.89 & 8.45 & 10.5 & 56.8 & 11.484 & 14.084 & 17.500 & 94.669 & 1.0601 & 1.1487 & 1.2430 & 1.9762 \\
\hline cst08 & cp051 & 2 & 17 & 9.37 & 9.53 & 21.3 & 63 & 15.617 & 15.884 & 35.501 & 105.002 & 1.1936 & 1.2010 & 1.5502 & 2.0212 \\
\hline cst12 & cp006 & 2 & 18 & 7.8 & 9.14 & 9.92 & 62.5 & 13.000 & 15.234 & 16.534 & 104.169 & 1.1140 & 1.1828 & 1.2184 & 2.0177 \\
\hline cst16 & cp105 & 2 & 19 & 6.31 & 7.85 & 21.7 & 60.8 & 10.517 & 13.084 & 36.167 & 101.335 & 1.0219 & 1.1167 & 1.5583 & 2.0058 \\
\hline cst $05 \mathrm{clc}$ & cp038 & 2 & 20 & 9.5 & 9.4 & 22.6 & 73.1 & 15.834 & 15.667 & 37.667 & 121.836 & 1.1996 & 1.1950 & 1.5760 & 2.0858 \\
\hline std & std-b2-3 & 2 & 21 & 19.4 & 9.22 & 77.1 & 50.1 & 19.400 & 9.220 & 77.100 & 50.100 & 1.2878 & 0.9647 & 1.8871 & 1.6998 \\
\hline std & std-b3-1 & 3 & 1 & 19.7 & 9.88 & 78.6 & 48.9 & 19.700 & 9.880 & 78.600 & 48.900 & 1.2945 & 0.9948 & 1.8954 & 1.6893 \\
\hline cst10 & cp095 & 3 & 2 & 7.31 & 8.66 & 10.9 & 56.2 & 12.184 & 14.434 & 18.167 & 93.669 & 1.0858 & 1.1594 & 1.2593 & 1.9716 \\
\hline $\mathrm{cst} 05 \mathrm{clc}$ & cp063 & 3 & 3 & 9.32 & 9.66 & 23 & 66 & 15.534 & 16.100 & 38.334 & 110.002 & 1.1913 & 1.2068 & 1.5836 & 2.0414 \\
\hline cst01 & cp041 & 3 & 4 & 8.33 & 9.23 & 18.5 & 58.5 & 13.884 & 15.384 & 30.834 & 97.502 & 1.1425 & 1.1871 & 1.4890 & 1.9890 \\
\hline $\mathrm{cst} 11 \mathrm{clc}$ & cp009 & 3 & 5 & 5.83 & 8.09 & 9.32 & 51.9 & 9.717 & 13.484 & 15.534 & 86.502 & 0.9875 & 1.1298 & 1.1913 & 1.9370 \\
\hline $\operatorname{cst} 12$ & cp049 & 3 & 6 & 8.13 & 9.63 & 10.6 & 61.4 & 13.550 & 16.050 & 17.667 & 102.335 & 1.1319 & 1.2055 & 1.2472 & 2.0100 \\
\hline $\operatorname{cst} 08$ & cp091 & 3 & 7 & 9.46 & 9.62 & 21.1 & 61.1 & 15.767 & 16.034 & 35.167 & 101.835 & 1.1977 & 1.2050 & 1.5461 & 2.0079 \\
\hline cst05 & cp068 & 3 & 8 & 9.38 & 10.2 & 22 & 66.1 & 15.634 & 17.000 & 36.667 & 110.169 & 1.1941 & 1.2305 & 1.5643 & 2.0421 \\
\hline cst08clc & cp100 & 3 & 9 & 7.63 & 8.38 & 19.6 & 60.2 & 12.717 & 13.967 & 32.667 & 100.335 & 1.1044 & 1.1451 & 1.5141 & 2.0015 \\
\hline cst16clc & $\mathrm{cp032}$ & 3 & 10 & 8.42 & 8.92 & 22.5 & 62.4 & 14.034 & 14.867 & 37.501 & 104.002 & 1.1472 & 1.1722 & 1.5740 & 2.0170 \\
\hline std & std-b3-2 & 3 & 11 & 19.6 & 9.64 & 77.2 & 48.7 & 19.600 & 9.640 & 77.200 & 48.700 & 1.2923 & 0.9841 & 1.8876 & 1.6875 \\
\hline cst01clc & cp080 & 3 & 12 & & & & & & & & & . & - & . & \\
\hline cst10clc & cp062 & 3 & 13 & 6.1 & 7.49 & 10.7 & 55.5 & 10.167 & 12.484 & 17.834 & 92.502 & 1.0072 & 1.0963 & 1.2512 & 1.9662 \\
\hline cst $03 \mathrm{clc}$ & cp083 & 3 & 14 & 8.69 & 9.1 & 17.5 & 68.6 & 14.484 & 15.167 & 29.167 & 114.336 & 1.1609 & 1.1809 & 1.4649 & 2.0582 \\
\hline cst17clc & cp010 & 3 & 15 & 8.38 & 8.72 & 22 & 61.9 & 13.967 & 14.534 & 36.667 & 103.169 & 1.1451 & 1.1624 & 1.5643 & 2.0135 \\
\hline $\operatorname{cst} 17$ & cp090 & 3 & 16 & 8.82 & 9.57 & 22.4 & 65.4 & 14.700 & 15.950 & 37.334 & 109.002 & 1.1673 & 1.2028 & 1.5721 & 2.0374 \\
\hline cst $12 \mathrm{clc}$ & cp099 & 3 & 17 & 8.12 & 8.93 & 11.7 & 63.2 & 13.534 & 14.884 & 19.500 & 105.335 & 1.1314 & 1.1727 & 1.2900 & 2.0226 \\
\hline cst11 & cp077 & 3 & 18 & 7.21 & 8.93 & 8.56 & 56.2 & 12.017 & 14.884 & 14.267 & 93.669 & 1.0798 & 1.1727 & 1.1543 & 1.9716 \\
\hline cst03 & cp043 & 3 & 19 & 8.93 & 10 & 16.9 & 68.7 & 14.884 & 16.667 & 28.167 & 114.502 & 1.1727 & 1.2219 & 1.4497 & 2.0588 \\
\hline cst16 & cp076 & 3 & 20 & 6.8 & 8.26 & 21.5 & 61.6 & 11.334 & 13.767 & 35.834 & 102.669 & 1.0544 & 1.1388 & 1.5543 & 2.0114 \\
\hline std & std-b3-3 & 3 & 21 & 21 & 10.1 & 79.9 & 53 & 21.000 & 10.100 & 79.900 & 53.000 & 1.3222 & 1.0043 & 1.9025 & 1.7243 \\
\hline
\end{tabular}

Notes:

(1). Values below the detection limit (indicated by a "<") were converted to $1 / 2$ the detection limit.

(2) The shaded entries indicate that the solution-weight fell outside of the guidelines for a successful PCT result. 
WSRC-TR-2001-00125

Revision 0

Table A.1: Composition of PCT Leachate Solutions (continued)

\begin{tabular}{|c|c|c|c|c|c|c|c|c|c|c|c|c|c|c|c|}
\hline \multirow{3}{*}{$\begin{array}{c}\text { Glass } \\
\text { ID } \\
\end{array}$} & \multirow{3}{*}{$\begin{array}{c}\text { Lab } \\
\text { ID } \\
\end{array}$} & \multirow[b]{3}{*}{ Blk } & \multirow[b]{3}{*}{ Seq } & \multirow{2}{*}{\multicolumn{4}{|c|}{ Concentrations in ppm }} & \multicolumn{4}{|c|}{ Concentrations in ppm } & \multirow{2}{*}{\multicolumn{4}{|c|}{ Common Logarithm of ppm Concentrations }} \\
\hline & & & & & & & & (aft & r correctir & for diluti & & & & & \\
\hline & & & & $\mathbf{B}$ & $\mathbf{L i}$ & $\mathbf{N a}$ & $\mathbf{S i}$ & \begin{tabular}{l|l}
$\mathbf{B}$ & $\mathbf{1}$ \\
\end{tabular} & $\mathbf{L i}$ & $\mathrm{Na}$ & $\mathbf{S i}$ & $\log [\mathbf{B}]$ & $\log [\mathrm{Li}]$ & $\log [\mathrm{Na}]$ & $\log [\mathrm{Si}]$ \\
\hline std & std-b4-1 & 4 & 1 & 19.3 & 10.3 & 79.5 & 47.9 & 19.300 & 10.300 & 79.500 & 47.900 & 1.2856 & 1.0128 & 1.9004 & 1.6803 \\
\hline cst14clc & $\mathrm{cp} 036$ & 4 & 2 & 6.66 & 8.93 & 19 & 61.5 & 11.100 & 14.884 & 31.667 & 102.502 & 1.0453 & 1.1727 & 1.5006 & 2.0107 \\
\hline cst15 & сp008 & 4 & 3 & 7.64 & 9.79 & 16.9 & 55.3 & 12.734 & 16.317 & 28.167 & 92.169 & 1.1050 & 1.2126 & 1.4497 & 1.9646 \\
\hline $\operatorname{cst} 06$ & cp075 & 4 & 4 & 8.16 & 9.35 & 21.2 & 53.3 & 13.600 & 15.584 & 35.334 & 88.835 & 1.1335 & 1.1927 & 1.5482 & 1.9486 \\
\hline cst04clc & $\mathrm{cp081}$ & 4 & 5 & 8.22 & 8.99 & 21.6 & 58.9 & 13.700 & 14.984 & 36.001 & 98.169 & 1.1367 & 1.1756 & 1.5563 & 1.9920 \\
\hline $\mathrm{cst} 07 \mathrm{clc}$ & cp042 & 4 & 6 & 11.1 & 11.4 & 21.7 & 78.6 & 18.500 & 19.000 & 36.167 & 131.003 & 1.2672 & 1.2788 & 1.5583 & 2.1173 \\
\hline cst14 & cp087 & 4 & 7 & 6.62 & 9.41 & 17.9 & 61.2 & 11.034 & 15.684 & 29.834 & 102.002 & 1.0427 & 1.1954 & 1.4747 & 2.0086 \\
\hline cst09clc & cp057 & 4 & 8 & 8.39 & 9.27 & 18.3 & 59.5 & 13.984 & 15.450 & 30.501 & 99.169 & 1.1456 & 1.1889 & 1.4843 & 1.9964 \\
\hline cst09 & cp001 & 4 & 9 & 8.73 & 10 & 17.8 & 59.8 & 14.550 & 16.667 & 29.667 & 99.669 & 1.1629 & 1.2219 & 1.4723 & 1.9986 \\
\hline cst $15 \mathrm{clc}$ & cp103 & 4 & 10 & 8.2 & 9.54 & 18.5 & 62.5 & 13.667 & 15.900 & 30.834 & 104.169 & 1.1357 & 1.2014 & 1.4890 & 2.0177 \\
\hline std & \begin{tabular}{|l|} 
std-b4-2 \\
\end{tabular} & 4 & 11 & 19.9 & 10.6 & 82 & 49.5 & 19.900 & 10.600 & 82.000 & 49.500 & 1.2989 & 1.0253 & 1.9138 & 1.6946 \\
\hline ARM & \begin{tabular}{|l} 
cp031 \\
\end{tabular} & 4 & 12 & 12.9 & 10 & 25.5 & 39.3 & 21.500 & 16.667 & 42.501 & 65.501 & 1.3324 & 1.2219 & 1.6284 & 1.8163 \\
\hline EA & cp047 & 4 & 13 & 17.2 & 6.71 & 49.1 & 30.2 & 286.667 & 111.834 & 818.335 & 503.334 & 2.4574 & 2.0486 & 2.9129 & 2.7019 \\
\hline $\mathrm{cst} 02 \mathrm{clc}$ & cp060 & 4 & 14 & 6.36 & 9.01 & 11.1 & 60.8 & 10.600 & 15.017 & 18.500 & 101.335 & 1.0253 & 1.1766 & 1.2672 & 2.0058 \\
\hline $\mathrm{cst} 06 \mathrm{clc}$ & $\mathrm{cp} 012$ & 4 & 15 & 6.75 & 8.09 & 20.3 & 53.8 & 11.250 & 13.484 & 33.834 & 89.668 & 1.0512 & 1.1298 & 1.5294 & 1.9526 \\
\hline cst07 & cp086 & 4 & 16 & 11.8 & 12.8 & 21.9 & 79.5 & 19.667 & 21.334 & 36.501 & 132.503 & 1.2937 & 1.3291 & 1.5623 & 2.1222 \\
\hline $\operatorname{cst} 02$ & cp066 & 4 & 17 & 6.02 & 9.12 & 9.33 & 57.5 & 10.034 & 15.200 & 15.550 & 95.835 & 1.0015 & 1.1819 & 1.1917 & 1.9815 \\
\hline cst04 & cp035 & 4 & 18 & 8.92 & 9.95 & 22.5 & 59.5 & 14.867 & 16.584 & 37.501 & 99.169 & 1.1722 & 1.2197 & 1.5740 & 1.9964 \\
\hline cst13 & cp039 & 4 & 19 & 7.71 & 9.63 & 15.3 & 57.7 & 12.850 & 16.050 & 25.501 & 96.169 & 1.1089 & 1.2055 & 1.4065 & 1.9830 \\
\hline $\mathrm{cst} 13 \mathrm{clc}$ & cp089 & 4 & 20 & 7.85 & 9.35 & 16.7 & 60.5 & 13.084 & 15.584 & 27.834 & 100.835 & 1.1167 & 1.1927 & 1.4446 & 2.0036 \\
\hline std & std-b4-3 & 4 & 21 & 19.5 & 10.4 & 80.1 & 48.1 & 19.500 & 10.400 & 80.100 & 48.100 & 1.2900 & 1.0170 & 1.9036 & 1.6821 \\
\hline std & \begin{tabular}{|l} 
std-b5-1 \\
\end{tabular} & 5 & 1 & 19.2 & 9.39 & 81 & 49.7 & 19.200 & 9.390 & 81.000 & 49.700 & 1.2833 & 0.9727 & 1.9085 & 1.6964 \\
\hline cst09 & \begin{tabular}{|c|}
$\mathrm{cp} 034$ \\
\end{tabular} & 5 & 2 & 8.52 & 9.57 & 17.3 & 61.8 & 14.200 & 15.950 & 28.834 & 103.002 & 1.1523 & 1.2028 & 1.4599 & 2.0128 \\
\hline cst $14 \mathrm{clc}$ & cp072 & 5 & 3 & 5.82 & 7.68 & 16.2 & 61.6 & 9.700 & 12.800 & 27.001 & 102.669 & 0.9868 & 1.1072 & 1.4314 & 2.0114 \\
\hline EA & cp014 & 5 & 4 & 16.7 & 5.71 & 49 & 31.3 & 278.334 & 95.167 & 816.668 & 521.668 & 2.4446 & 1.9785 & 2.9120 & 2.7174 \\
\hline cst13 & cp073 & 5 & 5 & 7.05 & 8.72 & 12.6 & 58.6 & 11.750 & 14.534 & 21.000 & 97.669 & 1.0700 & 1.1624 & 1.3222 & 1.9898 \\
\hline cst13clc & cp028 & 5 & 6 & 6.92 & 8.16 & 14 & 59.6 & 11.534 & 13.600 & 23.334 & 99.335 & 1.0620 & 1.1335 & 1.3680 & 1.9971 \\
\hline cst07clc & cp027 & 5 & 7 & 9.97 & 10.3 & 18.9 & 74.7 & 16.617 & 17.167 & 31.501 & 124.503 & 1.2206 & 1.2347 & 1.4983 & 2.0952 \\
\hline cst02 & cp040 & 5 & 8 & 5.22 & 8.13 & 6.18 & 57.8 & $\begin{array}{l}8.700 \\
\end{array}$ & 13.550 & 10.300 & 96.335 & 0.9395 & 1.1319 & 1.0128 & 1.9838 \\
\hline cst07 & $\mathrm{cp025}$ & 5 & 9 & 10.4 & 10.9 & 18.1 & 74.6 & 17.334 & 18.167 & 30.167 & 124.336 & 1.2389 & 1.2593 & 1.4795 & 2.0946 \\
\hline cst $15 \mathrm{clc}$ & cp093 & 5 & 10 & 6.83 & 7.87 & 14.8 & 58.2 & 11.384 & 13.117 & 24.667 & 97.002 & 1.0563 & 1.1178 & 1.3921 & 1.9868 \\
\hline std & \begin{tabular}{|l} 
std-b5-2 \\
\end{tabular} & 5 & 11 & 19.4 & 9.58 & 82.1 & 50.5 & 19.400 & 9.580 & 82.100 & 50.500 & 1.2878 & 0.9814 & 1.9143 & 1.7033 \\
\hline $\operatorname{cst} 15$ & \begin{tabular}{|c|}
$\mathrm{cp} 074$ \\
\end{tabular} & 5 & 12 & 7.42 & 8.82 & 14.3 & 60.8 & 12.367 & 14.700 & 23.834 & 101.335 & 1.0923 & 1.1673 & 1.3772 & 2.0058 \\
\hline cst14 & $\mathrm{cp} 101$ & 5 & 13 & 5.91 & 8.08 & 14.4 & 62.7 & 9.850 & 13.467 & 24.000 & 104.502 & 0.9934 & 1.1293 & 1.3802 & 2.0191 \\
\hline cst06 & cp061 & 5 & 14 & 7.43 & 8.13 & 18.4 & 54.3 & 12.384 & 13.550 & 30.667 & 90.502 & 1.0928 & 1.1319 & 1.4867 & 1.9567 \\
\hline $\mathrm{cst} 02 \mathrm{clc}$ & $\mathrm{cp} 050$ & 5 & 15 & 5.24 & 7.31 & 7.02 & 58.6 & 8.734 & 12.184 & 11.700 & 97.669 & 0.9412 & 1.0858 & 1.0682 & 1.9898 \\
\hline cst09clc & сp078 & 5 & 16 & 7.45 & 7.86 & 14.8 & 57.7 & 12.417 & 13.100 & 24.667 & 96.169 & 1.0940 & 1.1173 & 1.3921 & 1.9830 \\
\hline cst04 & cp058 & 5 & 17 & 8.19 & 8.59 & 19.1 & 60.3 & 13.650 & 14.317 & 31.834 & 100.502 & 1.1351 & 1.1559 & 1.5029 & 2.0022 \\
\hline $\mathrm{cst} 04 \mathrm{clc}$ & $\mathrm{cp013}$ & 5 & 18 & 7.53 & 7.9 & 18.8 & 59.1 & 12.550 & 13.167 & 31.334 & 98.502 & 1.0987 & 1.1195 & 1.4960 & 1.9934 \\
\hline ARM & $\mathrm{cp} 102$ & 5 & 19 & 12.2 & 8.91 & 23.2 & 40.1 & 20.334 & 14.850 & 38.667 & 66.835 & 1.3082 & 1.1717 & 1.5873 & 1.8250 \\
\hline cst06clc & cp023 & 5 & 20 & 5.87 & 6.7 & 16.5 & 53.2 & 9.784 & 11.167 & 27.501 & 88.668 & 0.9905 & 1.0479 & 1.4393 & 1.9478 \\
\hline std & \begin{tabular}{|l|} 
std-b5-3 \\
\end{tabular} & 5 & 21 & 19.2 & 9.46 & 81 & 50 & 19.200 & 9.460 & 81.000 & 50.000 & 1.2833 & 0.9759 & 1.9085 & 1.6990 \\
\hline std & \begin{tabular}{|l|} 
std-b6-1 \\
\end{tabular} & 6 & 1 & 20 & 10.3 & 81.9 & 51 & 20.000 & 10.300 & 81.900 & 51.000 & 1.3010 & 1.0128 & 1.9133 & 1.7076 \\
\hline cst13clc & сp024 & 6 & 2 & 7.57 & 8.97 & 16.5 & 62 & 12.617 & 14.950 & 27.501 & 103.335 & 1.1010 & 1.1747 & 1.4393 & 2.0142 \\
\hline $\mathrm{cst} 15 \mathrm{clc}$ & cp002 & 6 & 3 & 7.62 & 8.83 & 17.7 & 61.9 & 12.700 & 14.717 & 29.501 & 103.169 & 1.1038 & 1.1678 & 1.4698 & 2.0135 \\
\hline cst09 & cp005 & 6 & 4 & 9.02 & 10 & 18.1 & 65 & 15.034 & 16.667 & 30.167 & 108.336 & 1.1771 & 1.2219 & 1.4795 & 2.0348 \\
\hline cst06 & $\mathrm{cp} 026$ & 6 & 5 & 7.87 & 8.92 & 20.6 & 54.8 & 13.117 & 14.867 & 34.334 & 91.335 & 1.1178 & 1.1722 & 1.5357 & 1.9606 \\
\hline $\mathrm{cst} 14 \mathrm{clc}$ & cp094 & 6 & 6 & 6.62 & 8.82 & 18.5 & 67.1 & 11.034 & 14.700 & 30.834 & 111.836 & 1.0427 & 1.1673 & 1.4890 & 2.0486 \\
\hline blank & cp071 & 6 & 7 & $<0.150$ & $<0.040$ & $<0.100$ & $<0.790$ & 0.125 & 0.033 & 0.083 & 0.658 & -0.9031 & -1.4771 & -1.0792 & -0.1816 \\
\hline cst04 & $\mathrm{cp} 021$ & 6 & 8 & 8.88 & 9.62 & 22.1 & 63.7 & 14.800 & 16.034 & 36.834 & 106.169 & 1.1703 & 1.2050 & 1.5663 & 2.0260 \\
\hline cst09clc & cp107 & 6 & 9 & 7.92 & 8.66 & 17.7 & 60 & 13.200 & 14.434 & 29.501 & 100.002 & 1.1206 & 1.1594 & 1.4698 & 2.0000 \\
\hline cst13 & cp019 & 6 & 10 & 7.45 & 9.23 & 14.7 & 61.1 & 12.417 & 15.384 & 24.500 & 101.835 & 1.0940 & 1.1871 & 1.3892 & 2.0079 \\
\hline std & \begin{tabular}{|l} 
std-b6-2 \\
\end{tabular} & 6 & 11 & 20 & 10.1 & 80.8 & 51.2 & 20.000 & 10.100 & 80.800 & 51.200 & 1.3010 & 1.0043 & 1.9074 & 1.7093 \\
\hline EA & cp092 & 6 & 12 & 17.8 & 6.34 & 50 & 32.4 & 296.667 & 105.667 & 833.335 & 540.001 & 2.4723 & 2.0239 & 2.9208 & 2.7324 \\
\hline ARM & cp007 & 6 & 13 & 13.2 & 9.67 & 25.2 & 42.1 & 22.000 & 16.117 & 42.001 & 70.168 & 1.3424 & 1.2073 & 1.6233 & 1.8461 \\
\hline cst06clc & cp018 & 6 & 14 & 6.39 & 7.61 & 19.3 & 55.5 & $\begin{array}{ll}10.650 \\
\end{array}$ & 12.684 & 32.167 & 92.502 & 1.0274 & 1.1032 & 1.5074 & 1.9662 \\
\hline cst07clc & cp033 & 6 & 15 & 11.2 & 11.2 & 21.4 & 81.7 & 18.667 & 18.667 & 35.667 & 136.169 & 1.2711 & 1.2711 & 1.5523 & 2.1341 \\
\hline cst15 & cp079 & 6 & 16 & 8.1 & 9.68 & 16.9 & 65.1 & 13.500 & 16.134 & 28.167 & 108.502 & 1.1303 & 1.2077 & 1.4497 & 2.0354 \\
\hline cst07 & cp011 & 6 & 17 & 12.4 & 12.6 & 21.7 & 86.9 & 20.667 & 21.000 & 36.167 & 144.836 & 1.3153 & 1.3222 & 1.5583 & 2.1609 \\
\hline cst04clc & cp106 & 6 & 18 & 8.29 & 8.86 & 21.6 & 63.4 & 13.817 & 14.767 & 36.001 & 105.669 & 1.1404 & 1.1693 & 1.5563 & 2.0239 \\
\hline $\mathrm{cst} 02 \mathrm{clc}$ & cp017 & 6 & 19 & 5.74 & 8.14 & 9.75 & 61.3 & 9.567 & 13.567 & 16.250 & 102.169 & 0.9808 & 1.1325 & 1.2109 & 2.0093 \\
\hline cst02 & $\mathrm{cp004}$ & 6 & 20 & 6.06 & 9.1 & 9.33 & 63.6 & 10.100 & 15.167 & 15.550 & 106.002 & 1.0043 & 1.1809 & 1.1917 & 2.0253 \\
\hline cst14 & cp096 & 6 & 21 & 6.36 & 8.86 & 17.3 & 64.4 & 10.600 & 14.767 & 28.834 & 107.336 & 1.0253 & 1.1693 & 1.4599 & 2.0307 \\
\hline std & std-b6-3 & 6 & 22 & 20.1 & 9.99 & 79.3 & 51.3 & 20.100 & 9.990 & 79.300 & 51.300 & 1.3032 & 0.9996 & 1.8993 & 1.7101 \\
\hline
\end{tabular}

Notes:

(1). Values below the detection limit (indicated by a "<") were converted to $1 / 2$ the detection limit.

(3) The shaded entries indicate that the solution-weight fell outside of the guidelines for a successful PCT result. 
Exhibit A.1: Plots of the Leachate Concentrations in Analytical Sequence by Element

B (ppm) By Analytical Sequence with EA and Blanks

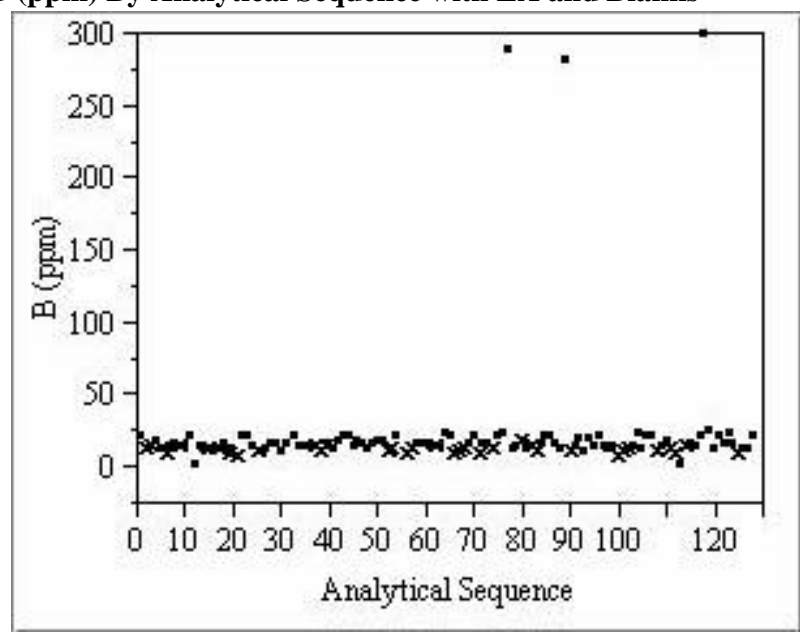

B (ppm) By Analytical Sequence without EA and Blanks

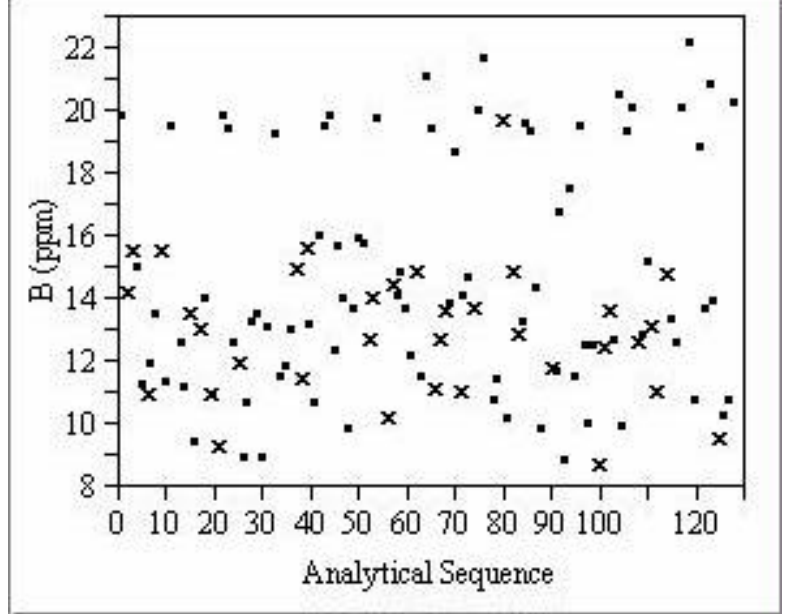

Li (ppm) By Analytical Sequence with EA and Blanks

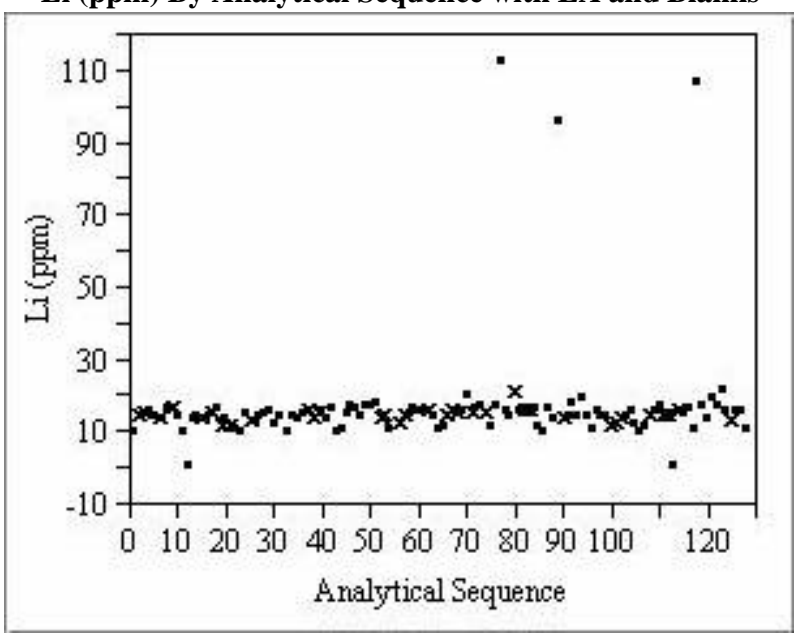

Li (ppm) By Analytical Sequence without EA and Blanks

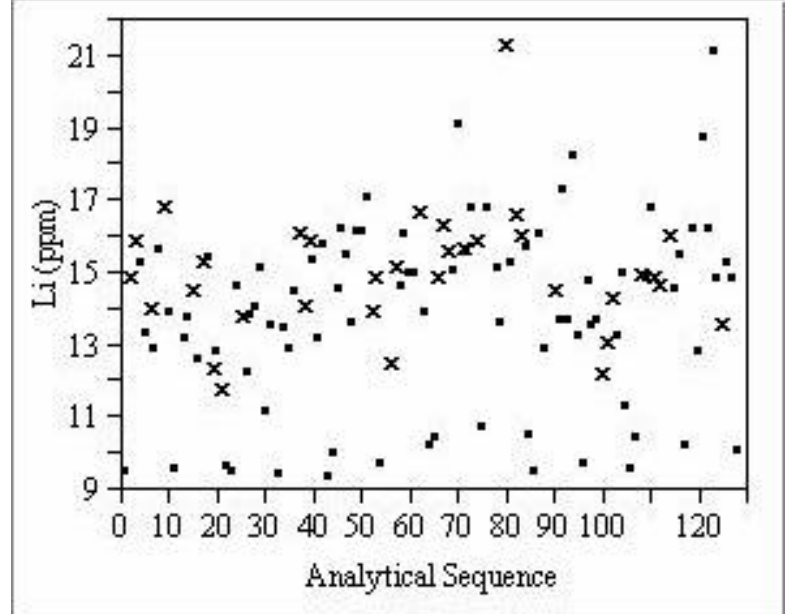


Exhibit A.1: Plots of the Leachate Concentrations in Analytical Sequence by Element

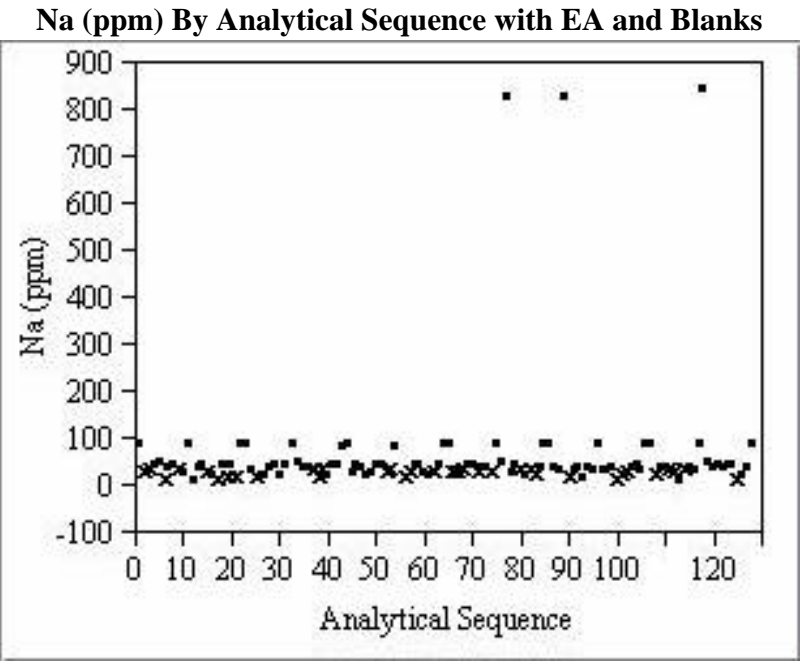

Na (ppm) By Analytical Sequence without EA and Blanks

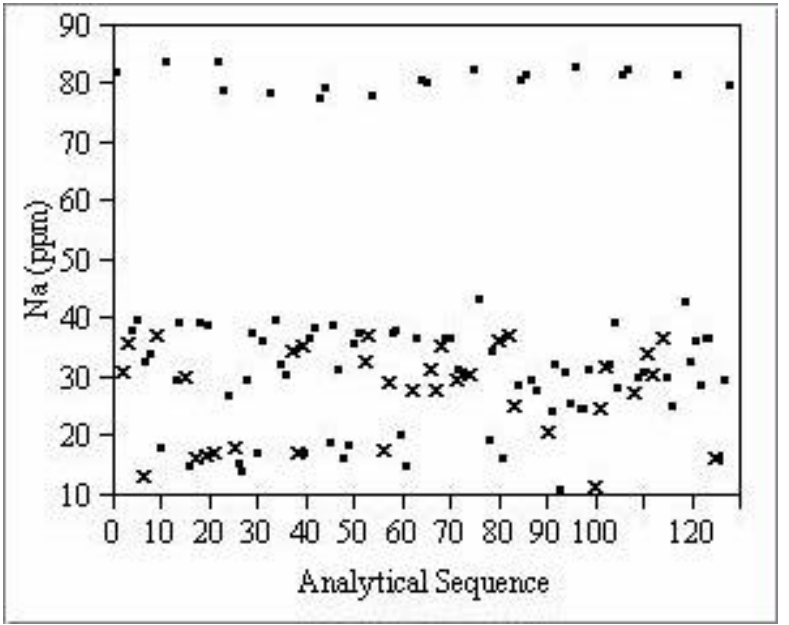

Si (ppm) By Analytical Sequence with EA and Blanks

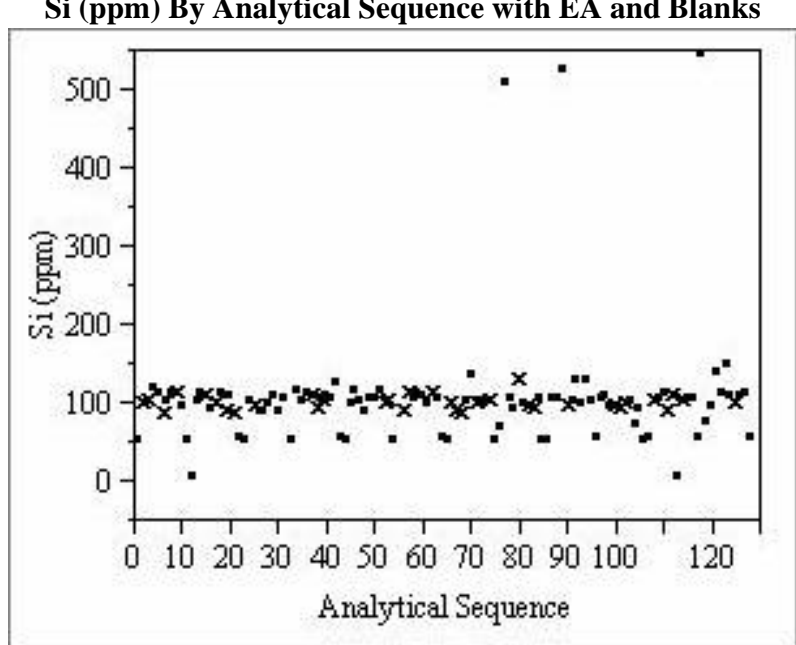

Si (ppm) By Analytical Sequence without EA and Blanks

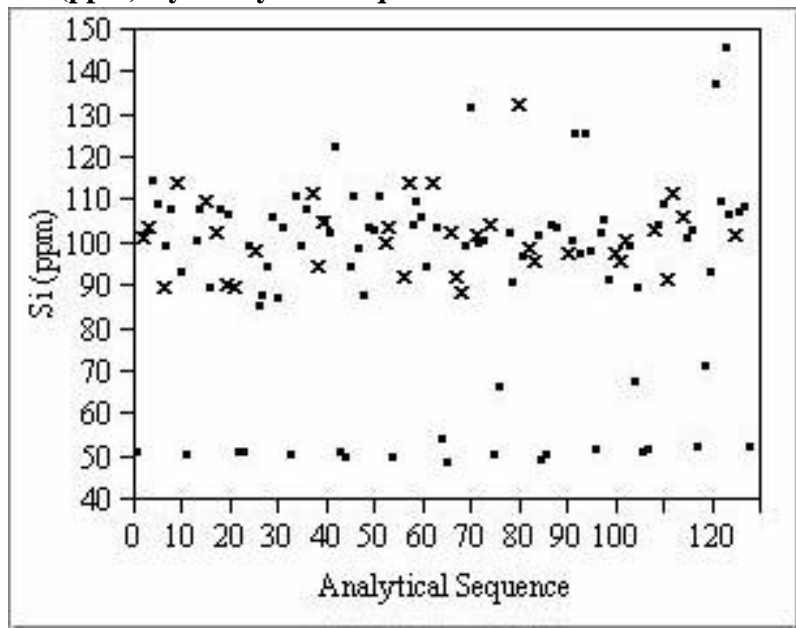


Exhibit A.2: Plots of the Leachate Concentrations by Glass ID by Element

\section{B (ppm) By Sample ID}
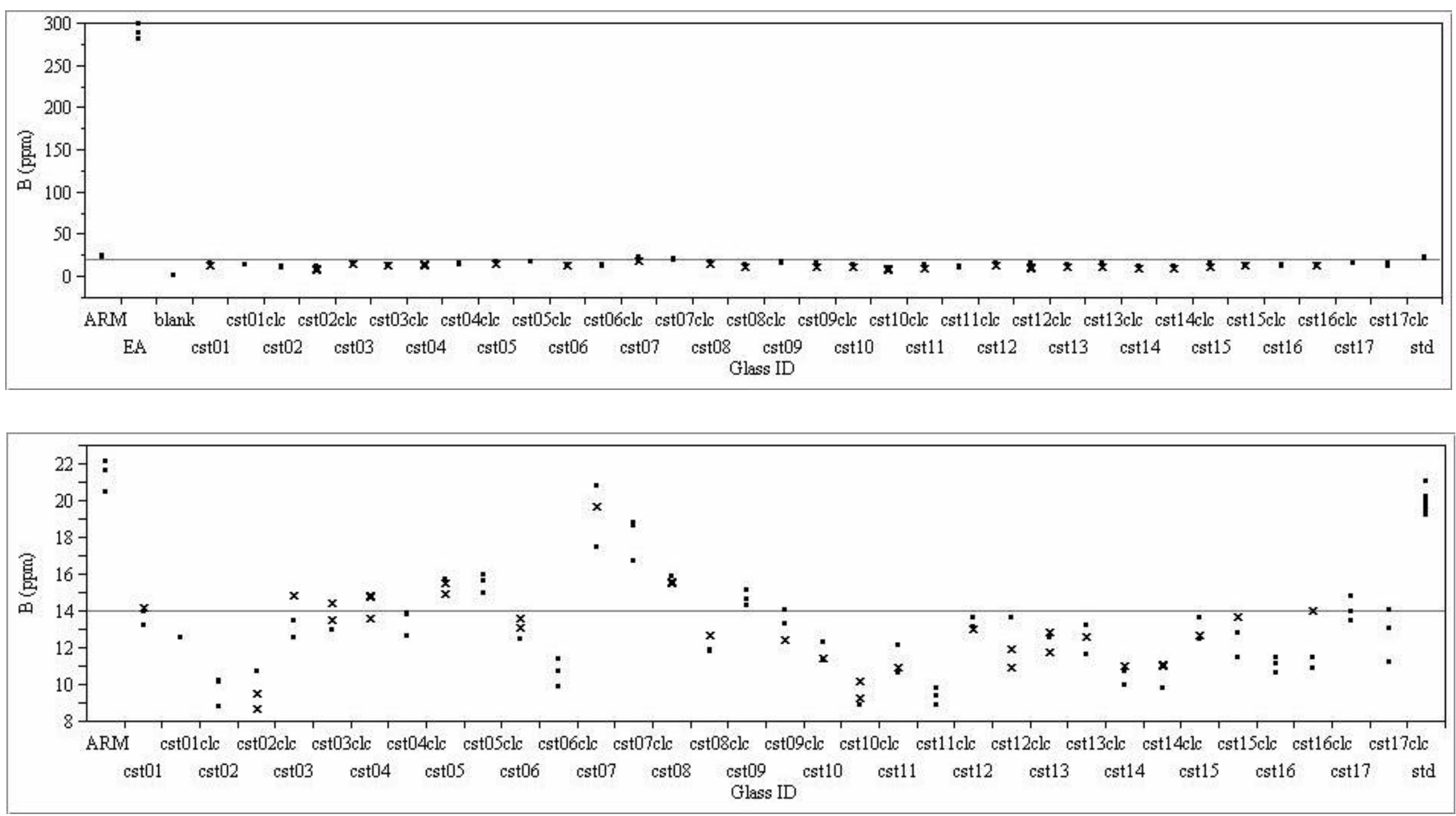


\section{Exhibit A.9: Plots of the Leachate Concentrations by Glass ID by Element (continued)}

\section{Li (ppm) By Sample ID}
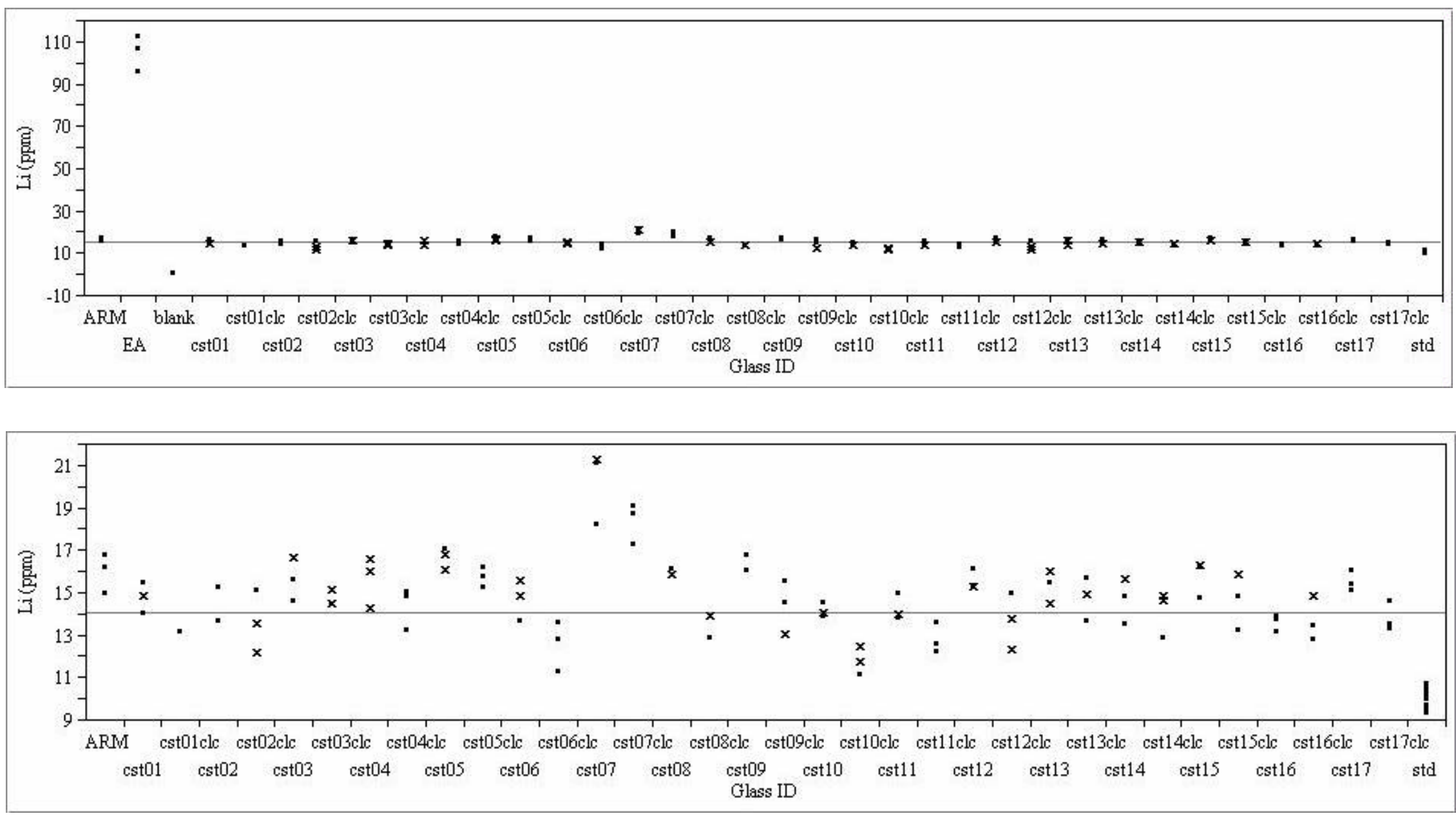


\section{Exhibit A.2: Plots of the Leachate Concentrations by Glass ID by Element (continued)}

\section{Na (ppm) By Sample ID}
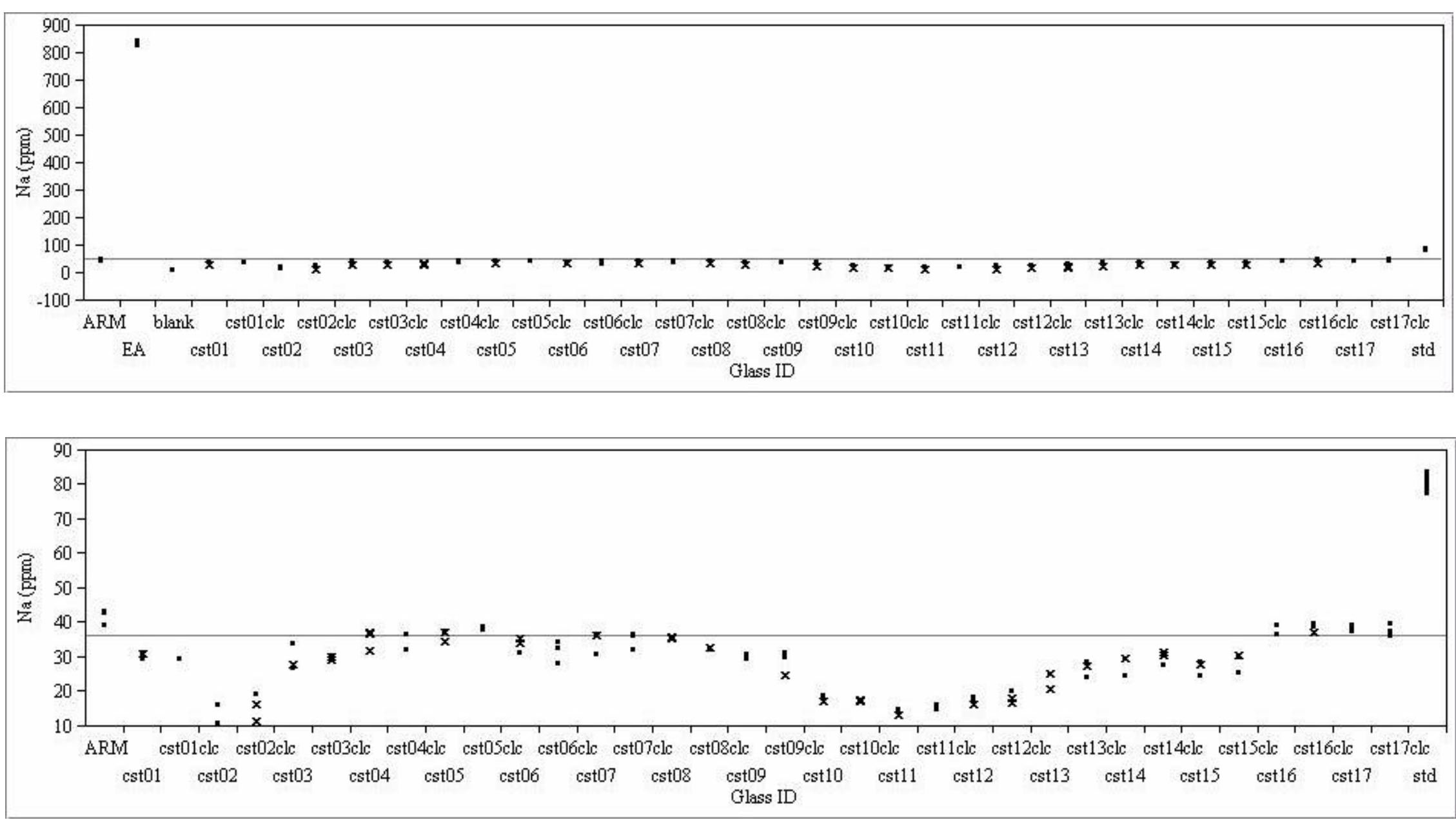


\section{Exhibit A.2: Plots of the Leachate Concentrations by Glass ID by Element (continued)}

\section{Si (ppm) By Sample ID}
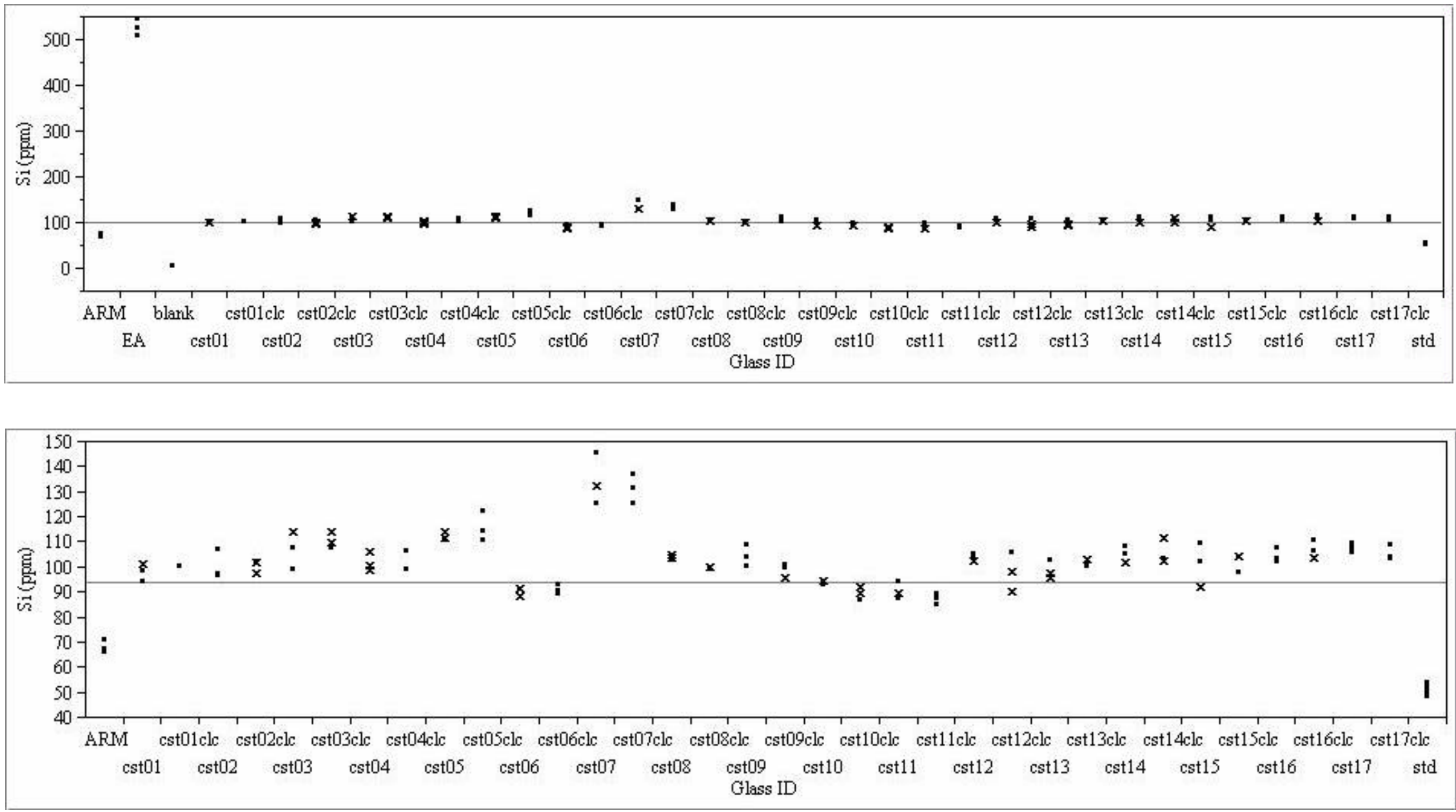
WSRC-TR-2001-00125

Revision 0

\section{Exhibit A.3: Plots of the Multi-Element Solution Standard by Element}

Oneway Analysis of B (ppm) By Block

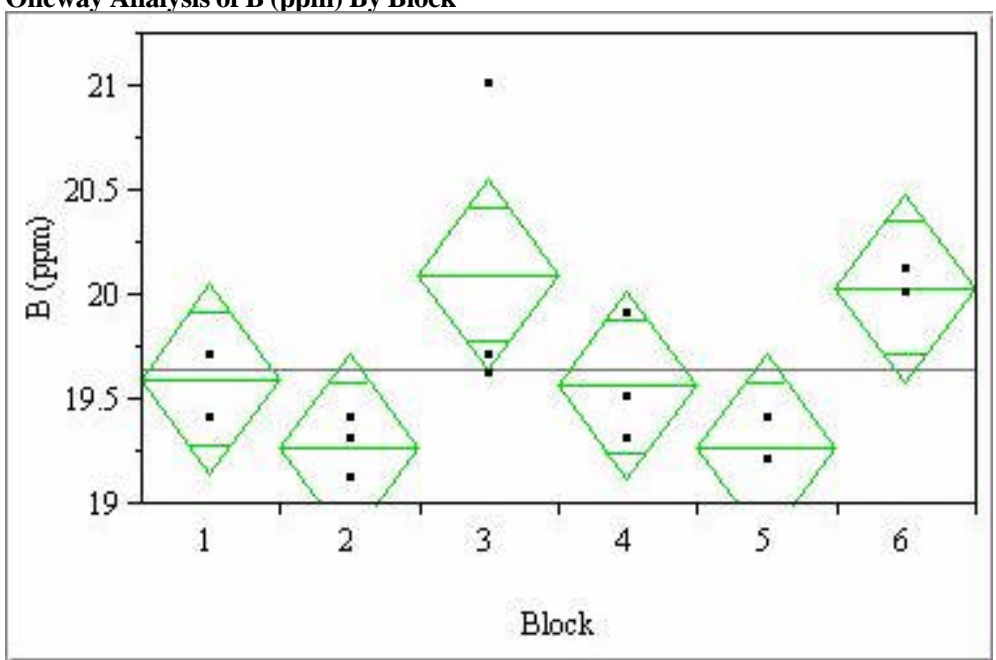

Oneway Anova

Summary of Fit

Rsquare

0.558446

Adj Rsquare

0.374465

$\begin{array}{ll}\text { Root Mean Square Error } & 0.359011\end{array}$

Mean of Response

19.63889
18

Observations (or Sum
Analysis of Variance

Source DF Sum of Squares Mean Square F Ratio Prob > F

$\begin{array}{lrrrrr}\text { Block } & 5 & 1.9561111 & 0.391222 & 3.0353 & 0.0534\end{array}$

$\begin{array}{lrr}\text { Error } & 12 & 1.5466667\end{array}$

0.128889

C. Total 17

3.5027778

Means for Oneway Anova

Level Number Mean Std Error Lower 95\% Upper 95\%

$\begin{array}{lrrrrr}\text { Level Number } & \text { Mean } & \text { Std Error } & \text { Lower 95\% } & \text { Upper 95\% } \\ 1 & 3 & 19.6000 & 0.20728 & 19.148 & 20.052\end{array}$

$\begin{array}{llllll}2 & 3 & 19.2667 & 0.20728 & 18.815 & 19.718 \\ 3 & 3 & 20.1000 & 0.20728 & 19.648 & 20.552\end{array}$

$\begin{array}{llllll}3 & 3 & 20.1000 & 0.20728 & 19.648 & 20.552 \\ 4 & 3 & 19.5667 & 0.20728 & 19.115 & 20.018 \\ 5 & 3 & 19.2667 & 0.20728 & 18.815 & 19.718\end{array}$

$\begin{array}{llllll}5 & 3 & 19.2667 & 0.20728 & 18.815 & 19.718 \\ 6 & 3 & 20.0333 & 0.20728 & 19.582 & 20.485\end{array}$

Std Error uses a pooled estimate of error variance
Oneway Analysis of Li (ppm) By Block

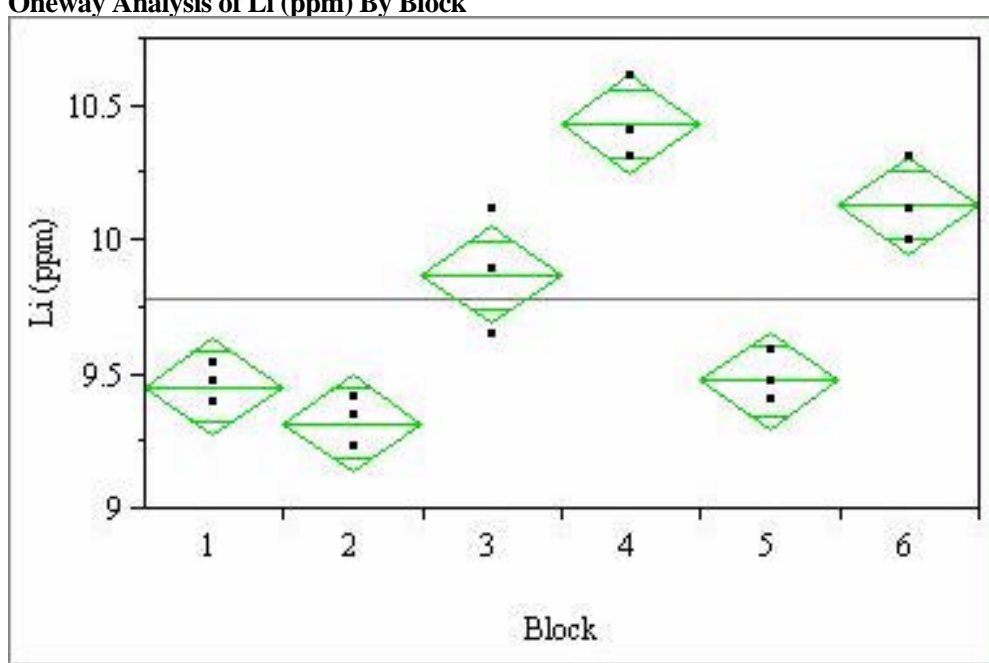

Oneway Anova

Summary of Fit

Adj Rsquare

0.921048
0.888151

$\begin{array}{ll}\text { Adj Rsquare } & 0.888151 \\ \text { Root Mean Square Error } & 0.143894\end{array}$

Mean of Response

9.781667

Observations (or Sum Wgts)

Analysis of Variance

Source DF Sum of Squares Mean Square F Ratio Prob > F

$\begin{array}{llllll}\text { Block } & 5 & 2.8985833 & 0.579717 & 27.9981 & <.0001\end{array}$

$\begin{array}{llll}\text { Error } & 12 & 0.2484667 & 0.020706\end{array}$

C. Total $17 \quad 3.1470500$

Means for Oneway Anova

Level Number Mean Std Error Lower 95\% Upper 95\%

$\begin{array}{lrrrrr}1 & 3 & 9.4567 & 0.08308 & 9.276 & 9.638\end{array}$

$\begin{array}{rrrrrr}2 & 3 & 9.3200 & 0.08308 & 9.139 & 9.501 \\ 3 & 3 & 9.8733 & 0.08308 & 9.692 & 10.054\end{array}$

$\begin{array}{rrrrrr}4 & 3 & 10.4333 & 0.08308 & 10.252 & 10.614 \\ 5 & 3 & 9.4767 & 0.08308 & 9.296 & 9.658\end{array}$

$\begin{array}{rrrrrr}5 & 3 & 9.4767 & 0.08308 & 9.296 & 9.658 \\ 6 & 3 & 10.1300 & 0.08308 & 9.949 & 10.311\end{array}$

Std Error uses a pooled estimate of error variance 
Exhibit A.3: Plots of the Multi-Element Solution Standard by Element (continued)

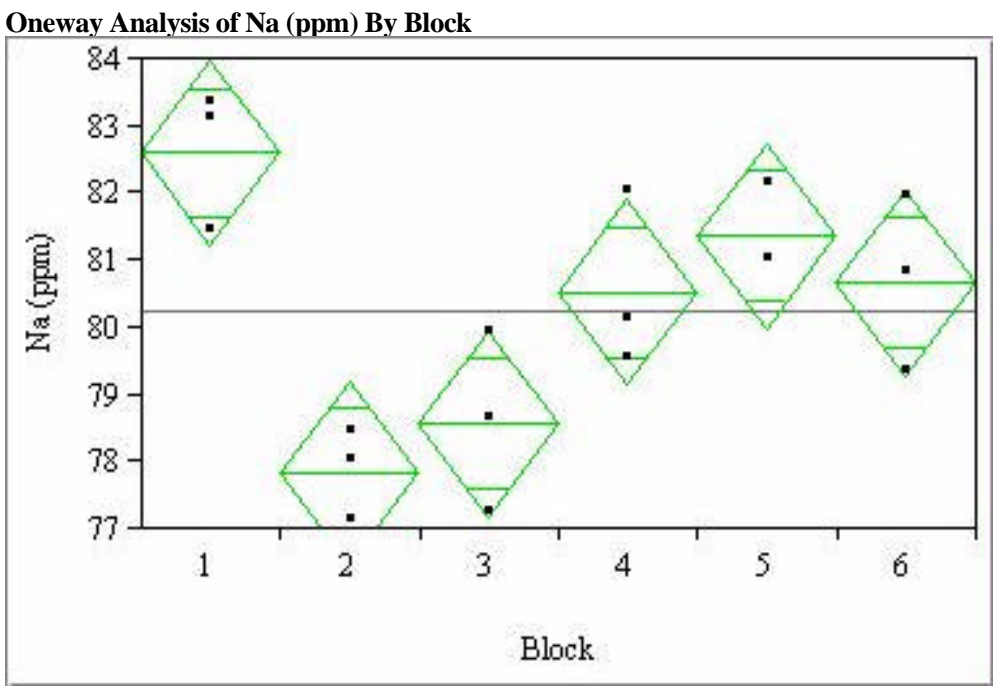

Oneway Anova

Summary of Fit

Rsquare

0.766645

Adj Rsquare

$\begin{array}{ll}\text { Adj Rsquare } & 0.669413 \\ \text { Root Mean Square Error } & 1.092906\end{array}$

Mean of Response

80.26111
18

Observations (or Sum Wg

Analysis of Variance

Source DF Sum of Squares Mean Square F Ratio Prob > F

$\begin{array}{lrrrrr}\text { Block } & 5 & 47.089444 & 9.41789 & 7.8847 & 0.0017\end{array}$

Error $\quad 12 \quad 14.333333$

1.19444

C. Total 17

61.422778

Means for Oneway Anova

Level Number Mean Std Error Lower 95\% Upper 95\%

$\begin{array}{lrrrrr}1 & 3 & 82.6000 & 0.63099 & 81.225 & 83.975\end{array}$

$\begin{array}{llllll}2 & 3 & 77.8333 & 0.63099 & 76.459 & 79.208 \\ 3 & 3 & 78.5667 & 0.63099 & 77.192 & 79.941\end{array}$

$\begin{array}{llllll}3 & 3 & 78.5667 & 0.63099 & 77.192 & 79.941 \\ 4 & 3 & 80.5333 & 0.63099 & 79.159 & 81.908 \\ 5 & 3 & 81.3667 & 0.63099 & 79.992 & 82.741\end{array}$

$\begin{array}{llllll}5 & 3 & 81.3667 & 0.63099 & 79.992 & 82.741 \\ 6 & 3 & 80.6667 & 0.63099 & 79.292 & 82.041\end{array}$

Std Error uses a pooled estimate of error variance
Oneway Analysis of Si (ppm) By Block

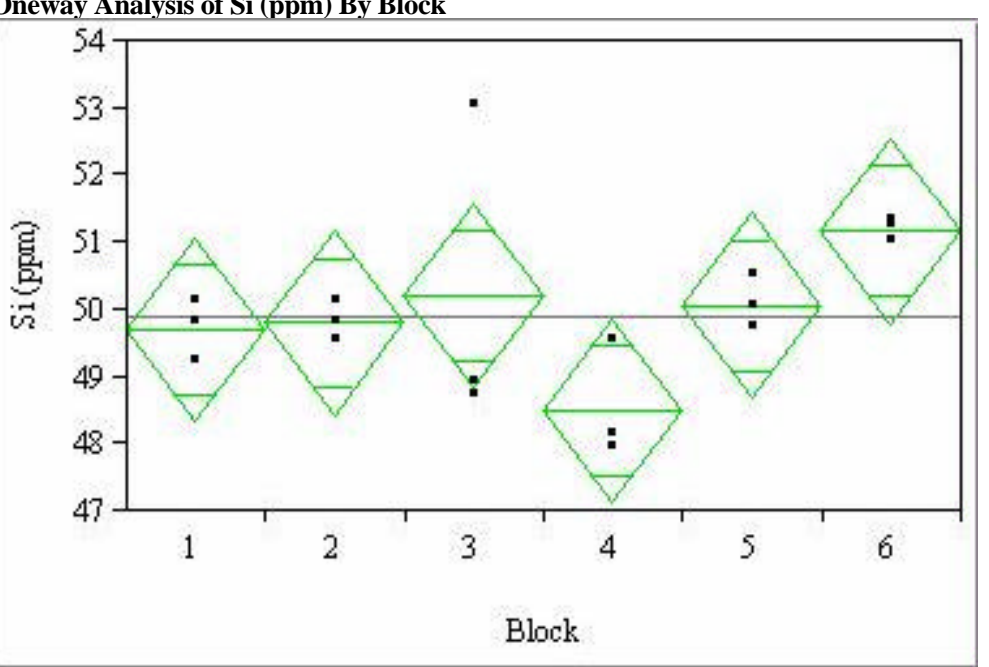

Oneway Anova

Summary of Fit

Rsquare

0.43959

Adj Rsquare

0.43959
0.206086

Root Mean Square

1.090617

Mean of Response

49.90556

Observations (or Sum Wgts)

Analysis of Variance

$\begin{array}{lrlrl}\text { Source DF } & \text { Sum of Squares Mean Square } \quad \text { F Ratio } & \text { Prob }>\text { F }\end{array}$

$\begin{array}{llllll}\text { Block } & 5 & 11.196111 & 2.23922 & 1.8826 & 0.1713\end{array}$

$\begin{array}{llll}\text { Error } & 12 & 14.273333 & 1.18944\end{array}$

$\begin{array}{lll}\text { C. Total } & 17 & 25.469444\end{array}$

Means for Oneway Anova

Level Number Mean Std Error Lower 95\% Upper 95\%

$\begin{array}{lrrrrr}1 & 3 & 49.7000 & 0.62967 & 48.328 & 51.072\end{array}$

$\begin{array}{llllll}2 & 3 & 49.8000 & 0.62967 & 48.428 & 51.172\end{array}$

$\begin{array}{llllll}3 & 3 & 50.2000 & 0.62967 & 48.828 & 51.572\end{array}$

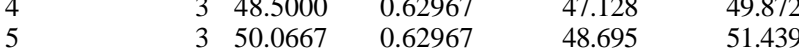

$\begin{array}{llllll}6 & 3 & 51.1667 & 0.62967 & 49.795 & 52.539\end{array}$

Std Error uses a pooled estimate of error variance 
Exhibit A.4: Correlations and Scatter Plots of the Normalized PCT's before Screening for Solution Weight Loss Problems

Measured Compositions - Linear Correlations \& Scatter Plots

\begin{tabular}{|c|c|c|c|c|}
\hline & $\log N L[B(g / L)]$ & $\log$ NL[Li $(\mathrm{g} / \mathrm{L})]$ & $\log \mathrm{NL}[\mathrm{Na}(\mathrm{g} / \mathrm{L})]$ & $\log$ NL[Si $(g / L)]$ \\
\hline $\log \mathbf{N L}[\mathrm{B}(\mathrm{g} / \mathrm{L})]$ & 1.0000 & 0.8015 & 0.7572 & 0.7496 \\
\hline $\log \mathrm{NL}[\mathrm{Li}(\mathrm{g} / \mathrm{L})]$ & 0.8015 & 1.0000 & 0.3791 & 0.5394 \\
\hline $\log \mathrm{NL}[\mathrm{Na}(\mathrm{g} / \mathrm{L})]$ & 0.7572 & 0.3791 & 1.0000 & 0.7194 \\
\hline $\log$ NL[Si $(\mathrm{g} / \mathrm{L})]$ & 0.7496 & 0.5394 & 0.7194 & 1.0000 \\
\hline
\end{tabular}

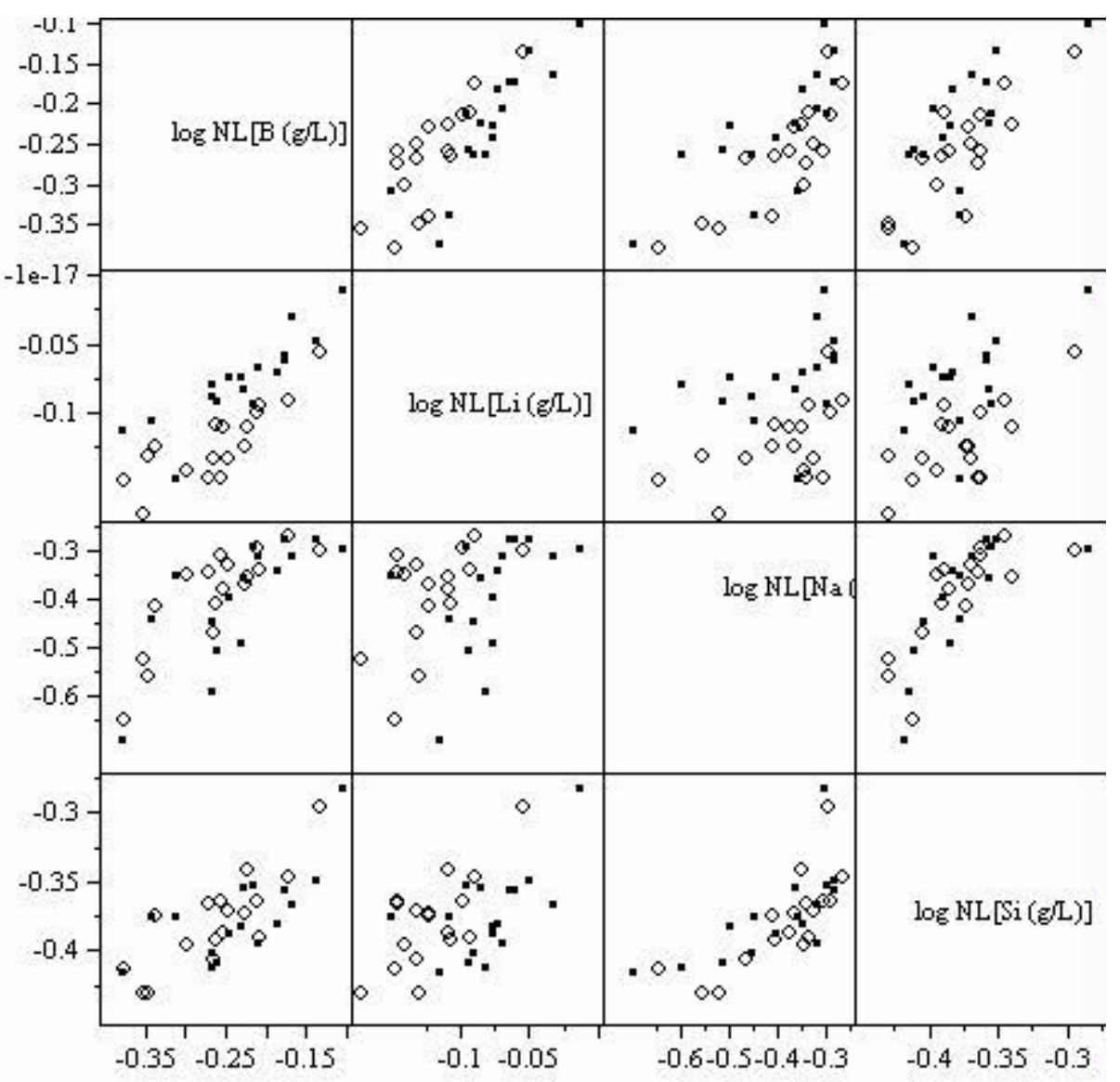


WSRC-TR-2001-00125

Revision 0

Exhibit A.4: Correlations and Scatter Plots of the Normalized PCT's before Screening for Solution Weight Loss Problems (continued)

Measured Bias-Corrected Compositions - Linear Correlations \& Scatter Plots

$\begin{array}{lcccc} & \log \mathbf{N L}[\mathbf{B}(\mathbf{g} / \mathbf{L})] & \log \mathbf{N L}[\mathbf{L i}(\mathbf{g} / \mathbf{L})] & \log \mathbf{N L}[\mathbf{N a}(\mathbf{g} / \mathbf{L})] & \log \mathbf{N L}[\mathbf{S i}(\mathbf{G} / \mathbf{L})] \\ \log \mathbf{N L}[\mathbf{B}(\mathbf{g} / \mathbf{L})] & 1.0000 & 0.7982 & 0.7610 & 0.7459 \\ \log \mathbf{N L}[\mathbf{L i}(\mathbf{g} / \mathbf{L})] & 0.7982 & 1.0000 & 0.3810 & 0.5303 \\ \log \mathbf{N L}[\mathbf{N a}(\mathbf{g} / \mathbf{L})] & 0.7610 & 0.3810 & 1.0000 & 0.7165 \\ \log \mathbf{N L}[\mathbf{S i}(\mathbf{G} / \mathbf{L})] & 0.7459 & 0.5303 & 0.7165 & 1.0000\end{array}$

\section{Scatterplot Matrix}

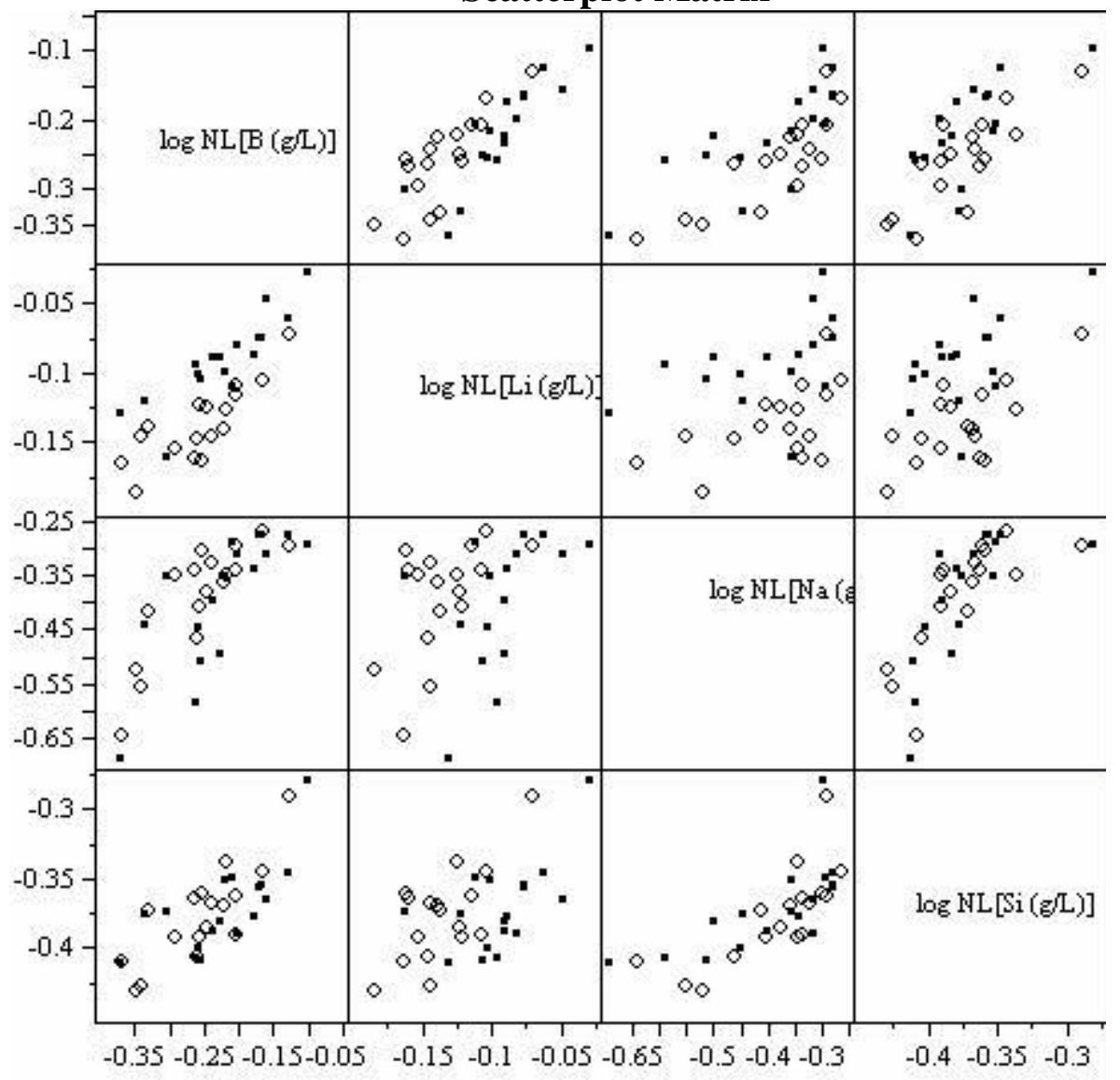


Exhibit A.4: Correlations and Scatter Plots of the Normalized PCT's before Screening for Solution Weight Loss Problems (continued)

\begin{tabular}{lcccc}
\multicolumn{5}{c}{ Target Compositions - Linear Correlations \& Scatter Plots } \\
& $\log \mathbf{N L}[\mathbf{B}(\mathbf{g} / \mathbf{L})]$ & $\log \mathbf{N L}[\mathbf{L i}(\mathbf{g} / \mathbf{L})]$ & $\log \mathbf{N L}[\mathbf{N a}(\mathbf{g} / \mathbf{L})]$ & $\log \mathbf{N L}[\mathbf{S i}(\mathbf{g} / \mathbf{L})]$ \\
$\log \mathbf{N L}[\mathbf{B}(\mathbf{g} / \mathbf{L})]$ & 1.0000 & 0.8116 & 0.7823 & 0.7935 \\
$\log \mathbf{N L}[\mathbf{L i}(\mathbf{g} / \mathbf{L})]$ & 0.8116 & 1.0000 & 0.4185 & 0.5723 \\
$\log \mathbf{N L}[\mathbf{N a}(\mathbf{g} / \mathbf{L})]$ & 0.7823 & 0.4185 & 1.0000 & 0.8280 \\
$\log \mathbf{N L}[\mathbf{S i}(\mathbf{g} / \mathbf{L})]$ & 0.7935 & 0.5723 & 0.8280 & 1.0000
\end{tabular}

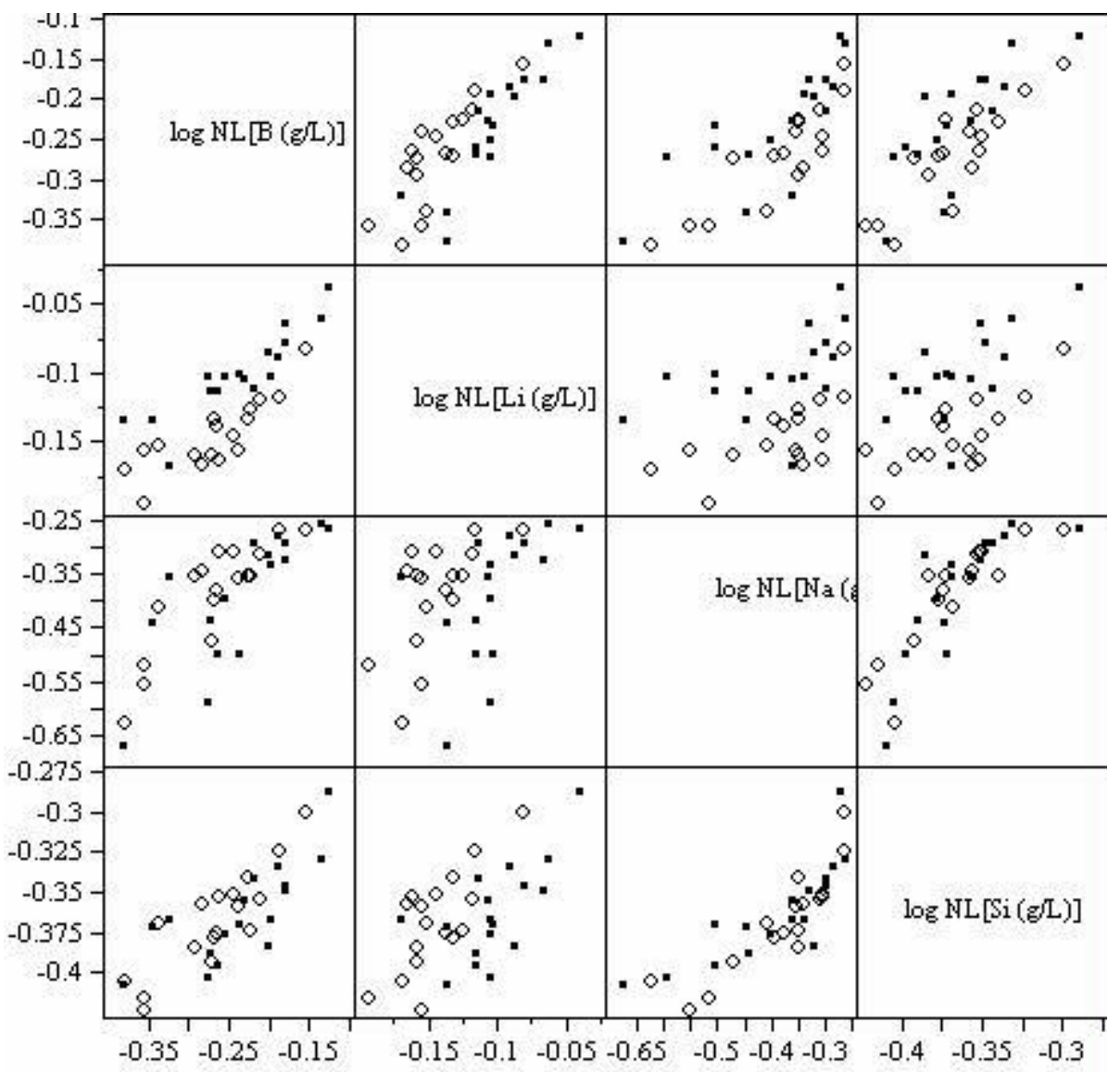


Revision 0

Exhibit A.5: Correlations and Scatter Plots of the Normalized PCTs after Screening for Solution Weight Loss Problems

\section{Measured Compositions}
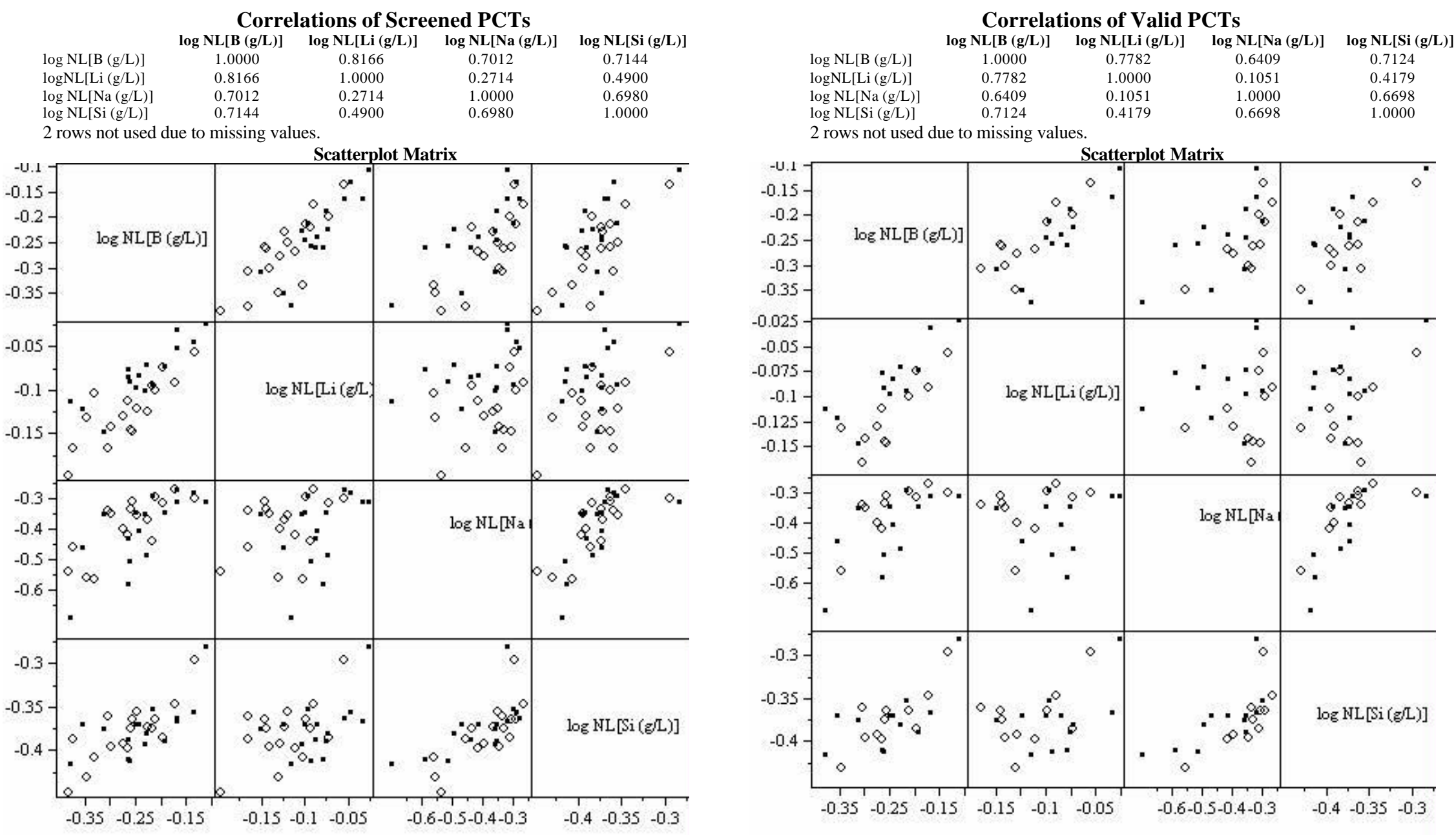
Revision 0

Exhibit A.5: Correlations and Scatter Plots of the Normalized PCTs after Screening for Solution Weight Loss Problems (continued)

Measured Bias-Corrected Compositions

Correlations of Screened PCTs

$\log N L[B(g / L)] \quad \log N L[L i(g / L)] \quad \log N L[N a(g / L)] \quad \log N L[S i(g / L)]$

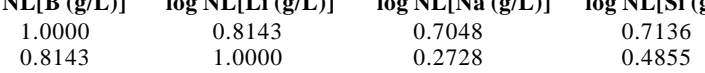

$\begin{array}{llll}1.8000 & 0.8143 & 0.7048 & 0.7136 \\ 0.7048 & 0.2728 & 0.2728 & 0.685 \\ 0.710000 & 1.0000 & 0.6969\end{array}$

$\log \mathrm{NL}[\mathrm{Li}(\mathrm{g} / \mathrm{L})]$

$\log \mathrm{NL}[\mathrm{Na}(\mathrm{g} / \mathrm{L})]$

0.7048

0.2728

1.0000

0.6969

2 rows not used due to missing values.

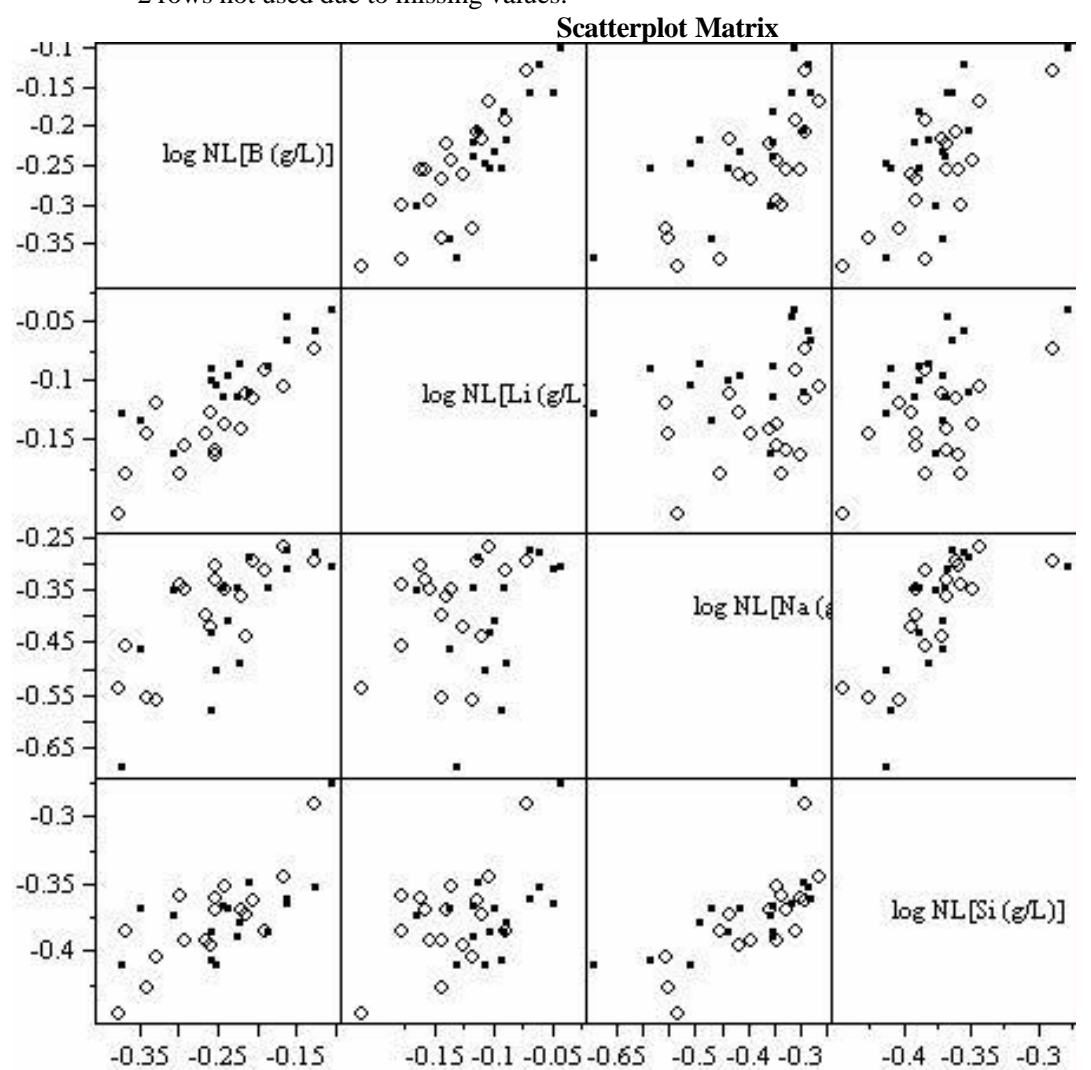

Correlations of Valid PCTs Correlations

\begin{tabular}{lcccc}
\multicolumn{7}{c}{$\begin{array}{c}\text { Correlations of Valid PCTs Correlations } \\
\\
\log \mathbf{N L}[\mathbf{B}(\mathrm{g} / \mathbf{L})]\end{array}$} & $\log \mathbf{N L}[\mathbf{L i}(\mathbf{g} / \mathbf{L})]$ & $\log \mathbf{N L}[\mathbf{N a}(\mathbf{g} / \mathbf{L})]$ & $\log \mathbf{N L}[\mathbf{S i}(\mathbf{g} / \mathbf{L})]$ \\
$\log \mathrm{NL}[\mathrm{B}(\mathrm{g} / \mathrm{L})]$ & 1.0000 & 0.7741 & 0.6437 & 0.7104 \\
$\operatorname{logNL}[\mathrm{Li}(\mathrm{g} / \mathrm{L})]$ & 0.7741 & 1.0000 & 0.1010 & 0.4122 \\
$\log \mathrm{NL}[\mathrm{Na}(\mathrm{g} / \mathrm{L})]$ & 0.6437 & 0.1010 & 1.0000 & 0.6671 \\
$\log \mathrm{NL}[\mathrm{Si}(\mathrm{g} / \mathrm{L})]$ & 0.7104 & 0.4122 & 0.6671 & 1.0000
\end{tabular}

2 rows not used due to missing values.

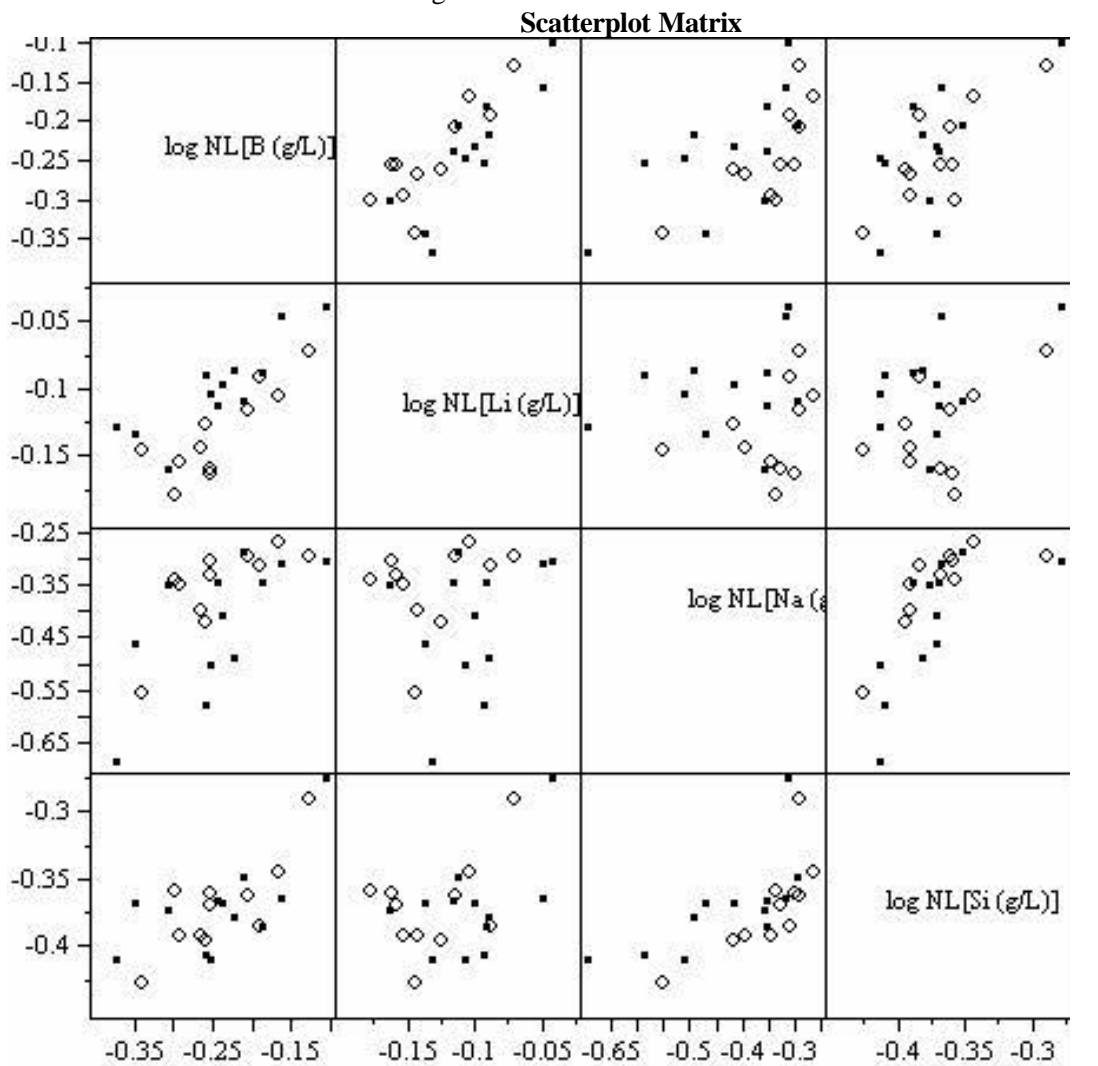


Revision 0

\section{Exhibit A.5: Correlations and Scatter Plots of the Normalized PCTs after Screening for Solution Weight Loss Problems (continued)}

\section{Targeted Compositions}
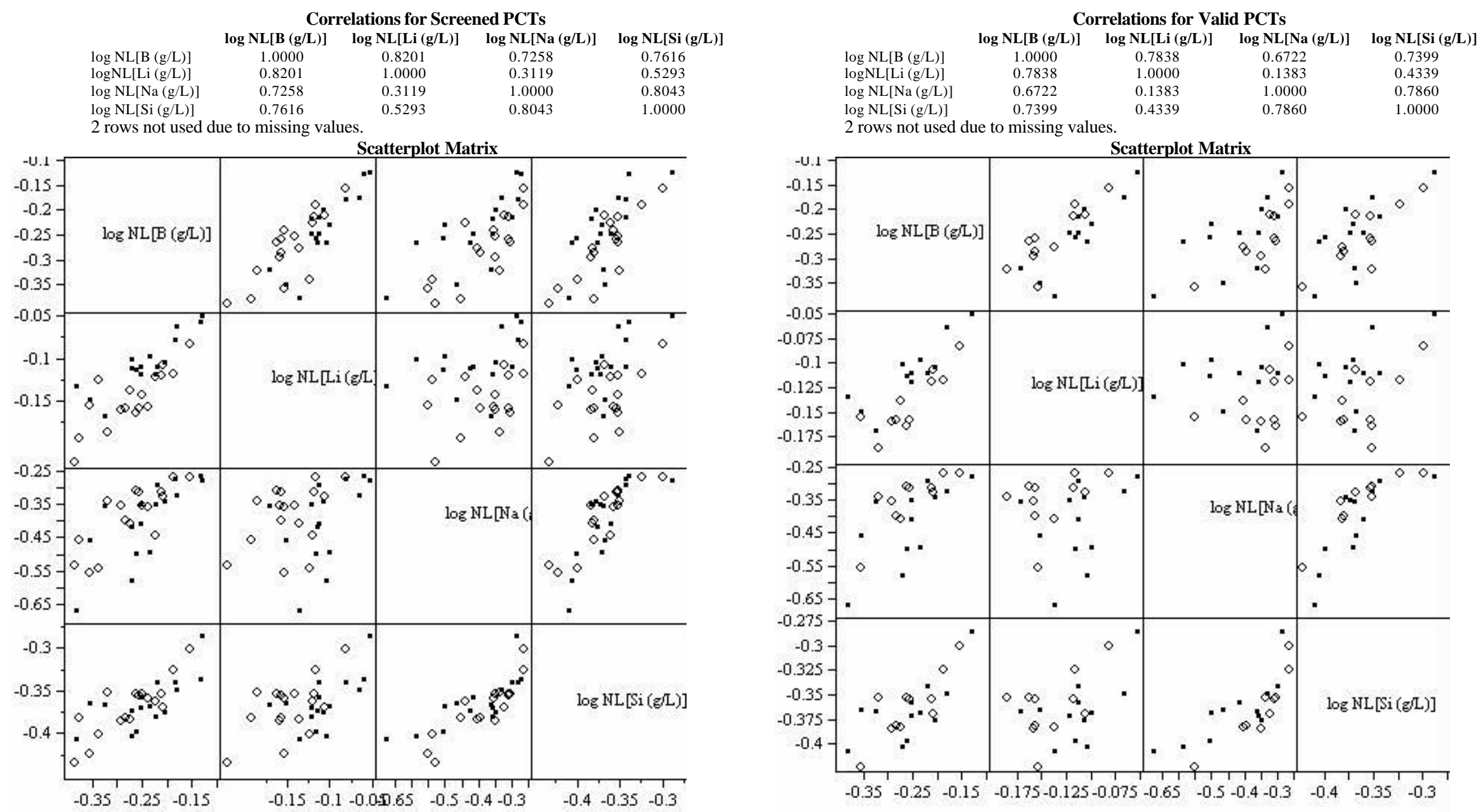
Exhibit A.6: Durability Model Predictions versus PCT Results Based Upon Measured Glass Compositions

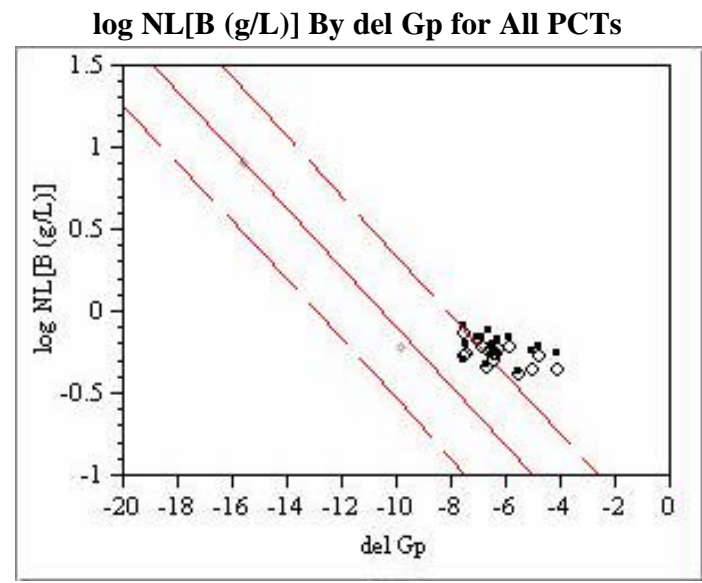

$\log$ NL[B (g/L)] By del Gp for Screened PCTs

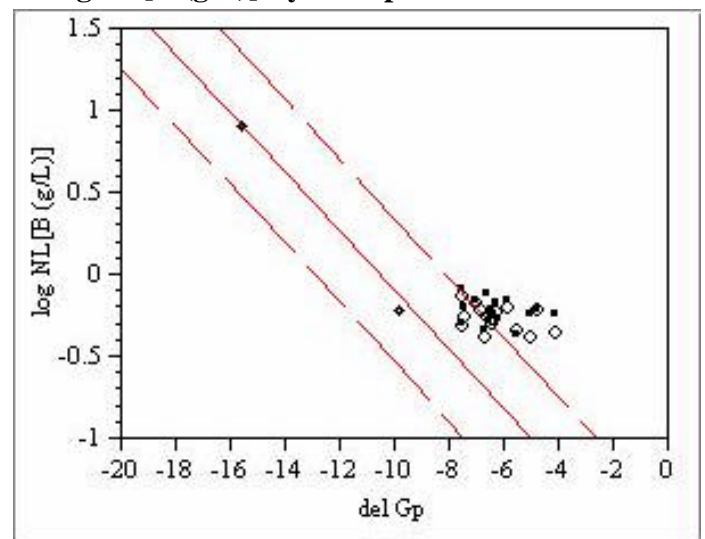

$\log$ NL[B (g/L)] By del Gp for Valid PCTs

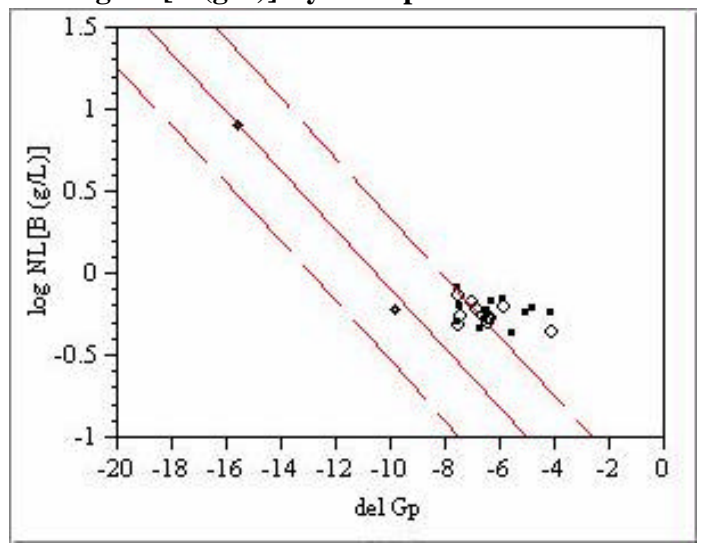

$\log \mathrm{NL}[\mathrm{Li}(\mathrm{g} / \mathrm{L})]$ By del Gp for All PCTs

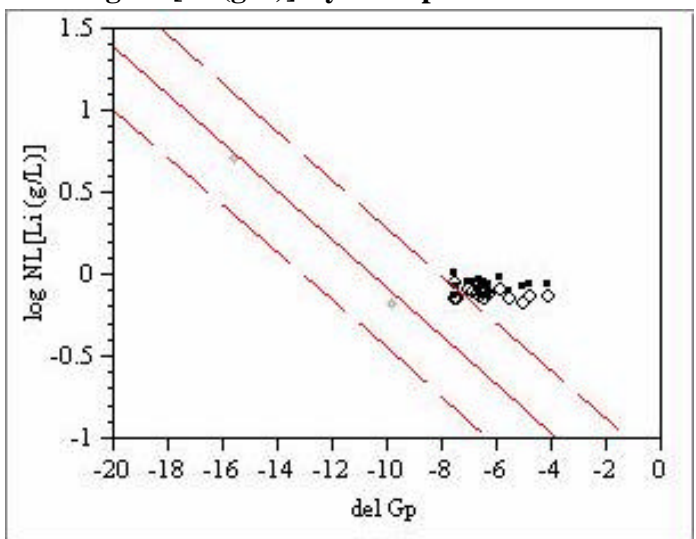

$\log$ NL[Li (g/L)] By del Gp for Screened PCTs

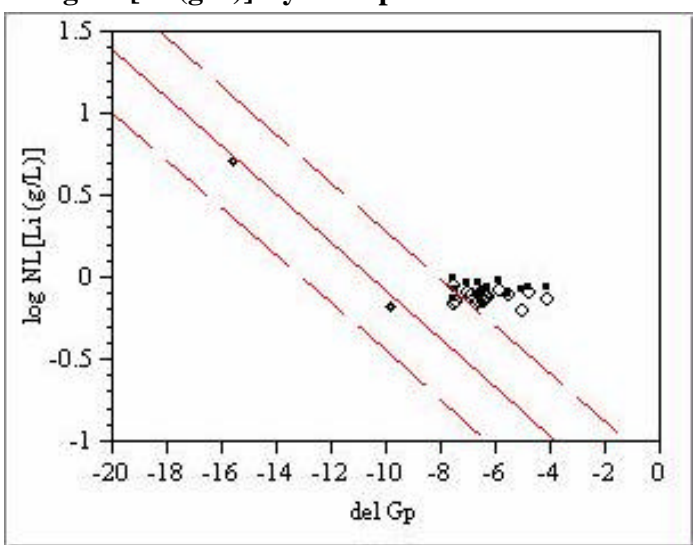

$\log \mathrm{NL}[\mathrm{Li}(\mathrm{g} / \mathrm{L})]$ By del Gp for Valid PCTs

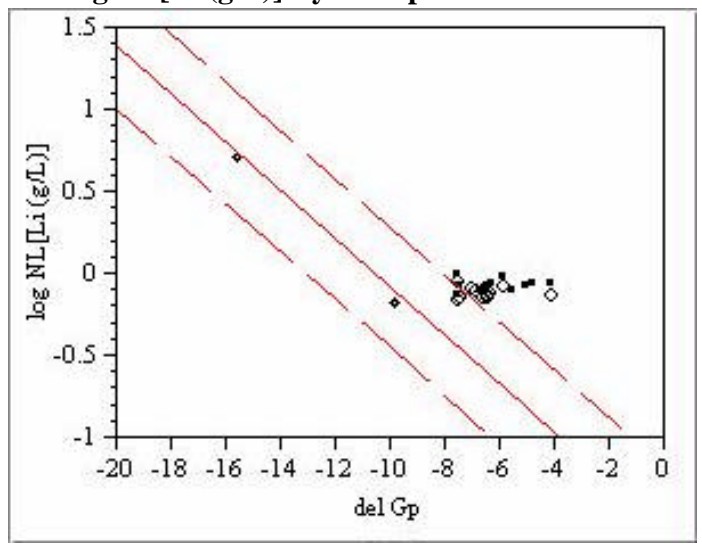


Exhibit A.6: Durability Model Predictions versus PCT Results Based Upon Measured Glass Compositions (continued)

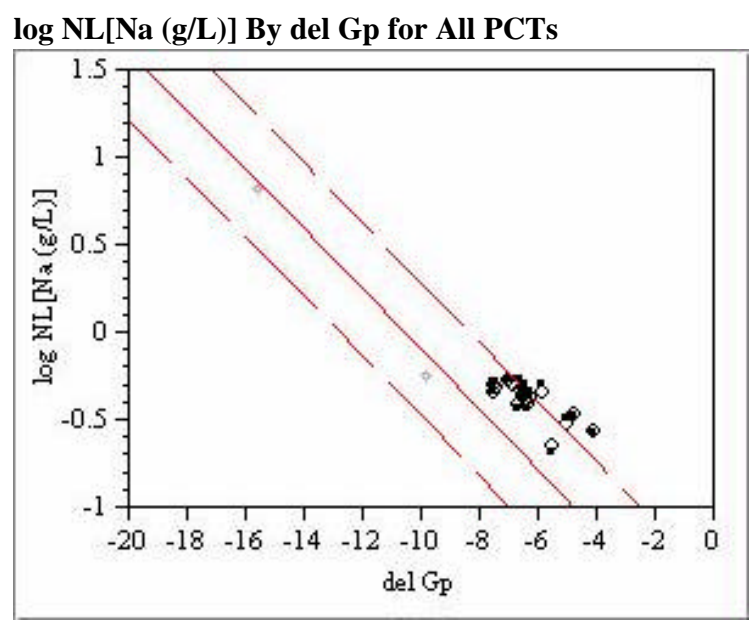

$\log \mathrm{NL}[\mathrm{Na}(\mathrm{g} / \mathrm{L})]$ By del Gp for Screened PCTs

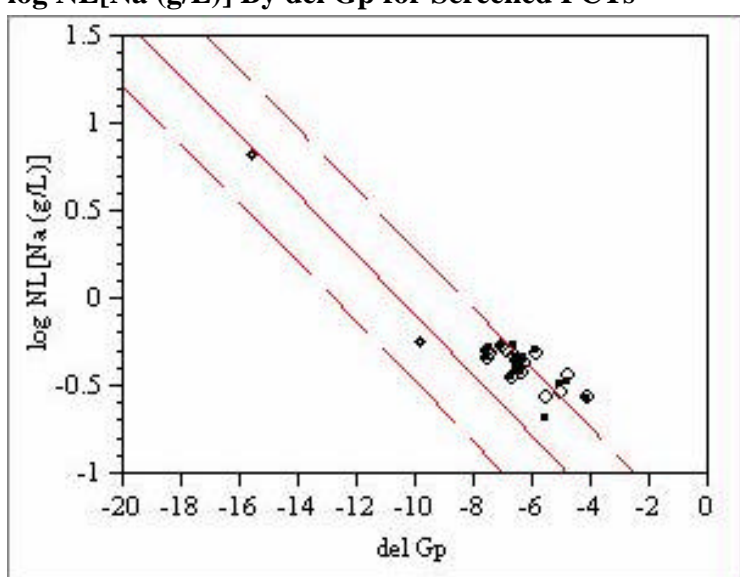

$\log$ NL[Na (g/L)] By del Gp for Valid PCTs

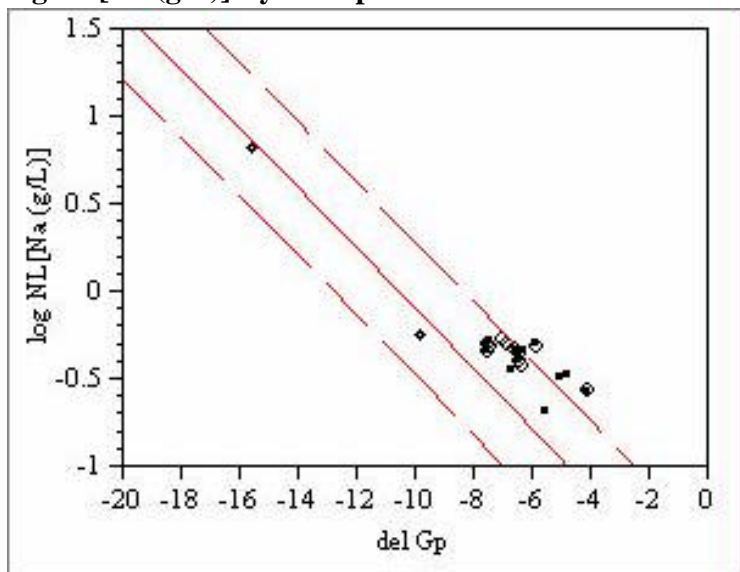

$\log$ NL[Si (g/L)] By del Gp for All PCTs

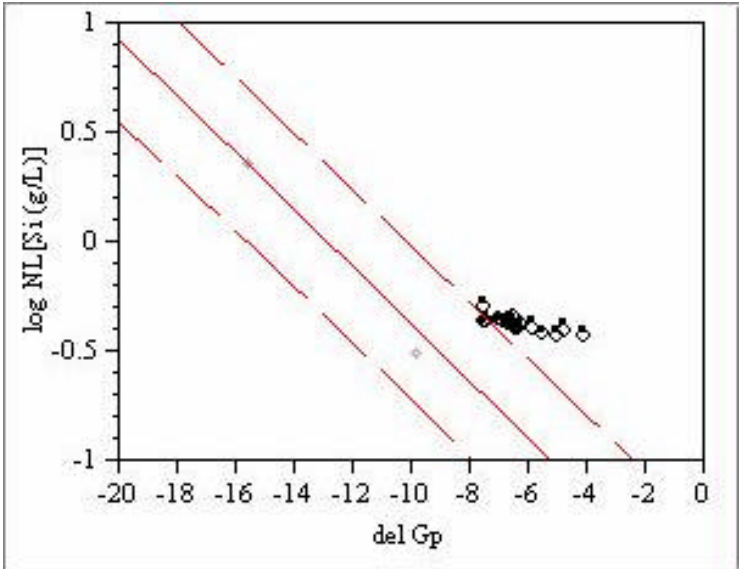

$\log$ NL[Si (g/L)] By del Gp for Screened PCTs

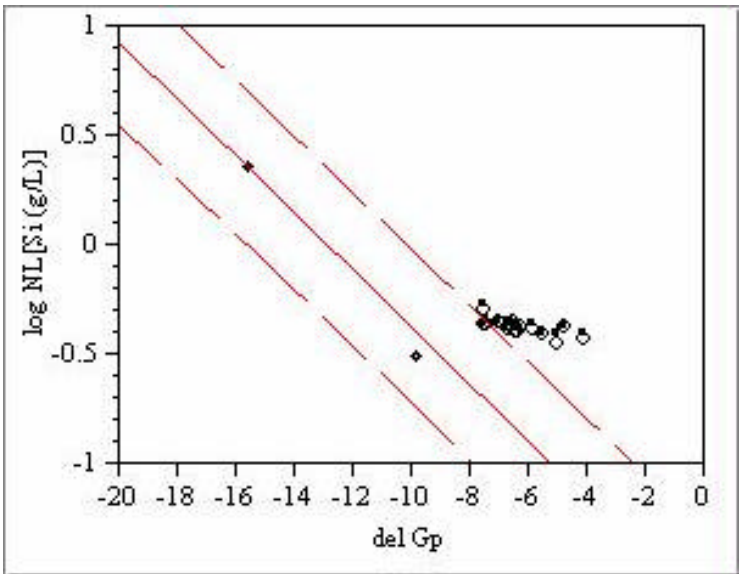

$\log$ NL[Si (g/L)] By del Gp for Valid PCTs

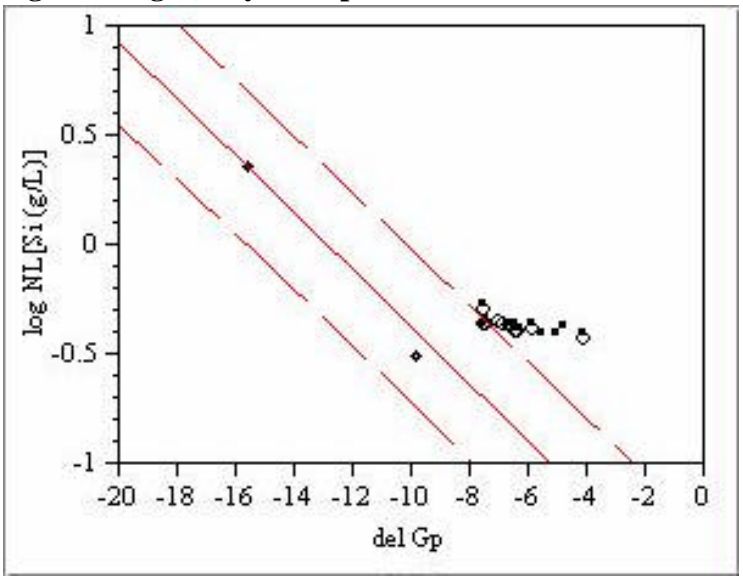


WSRC-TR-2001-00125

Revision 0

Exhibit A.6: Durability Model Predictions versus PCT Results

Based Upon Bias-Corrected, Measured Glass Compositions (continued)
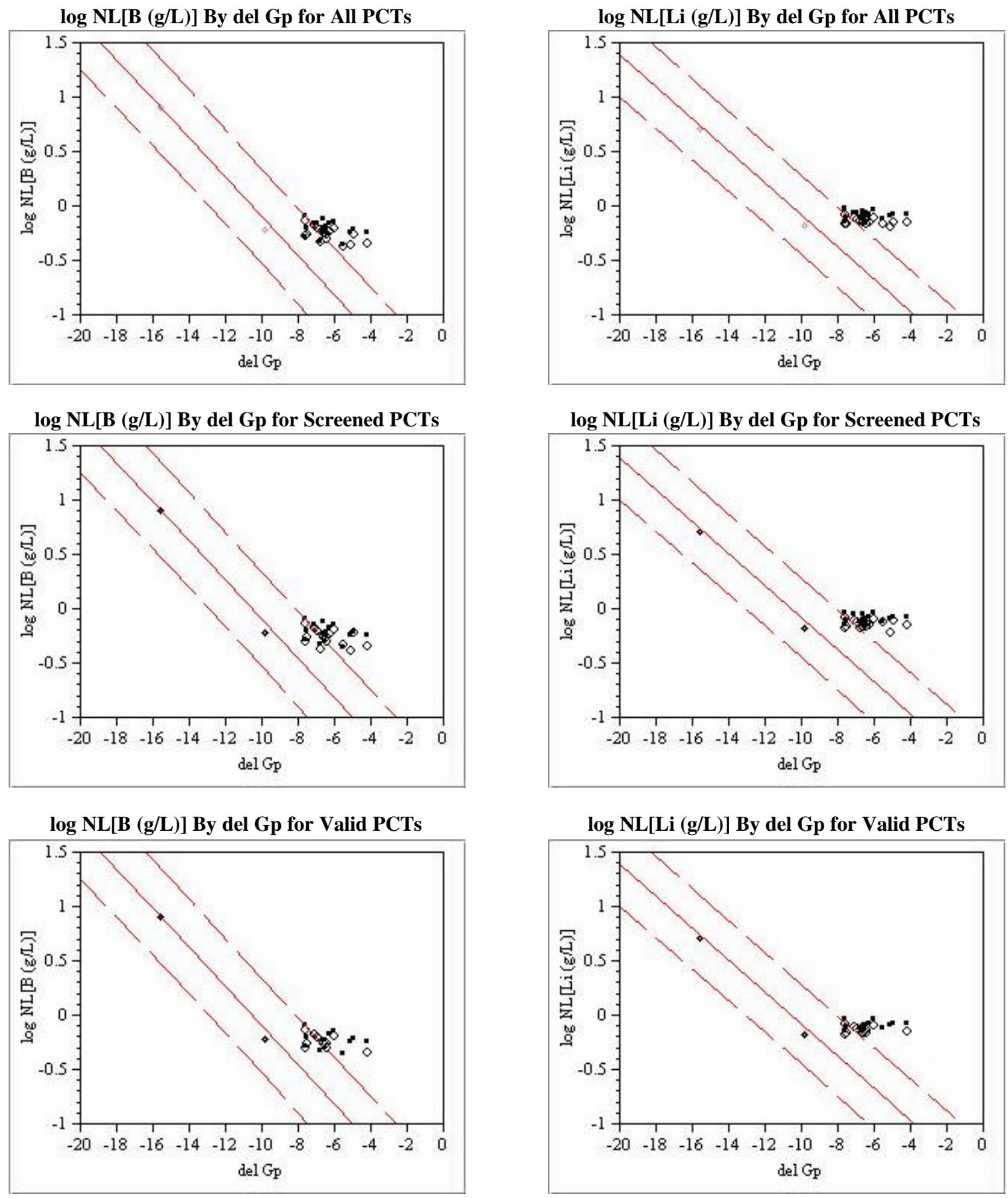
Exhibit A.6: Durability Model Predictions versus PCT Results

Based Upon Bias-Corrected, Measured Glass Compositions (continued)

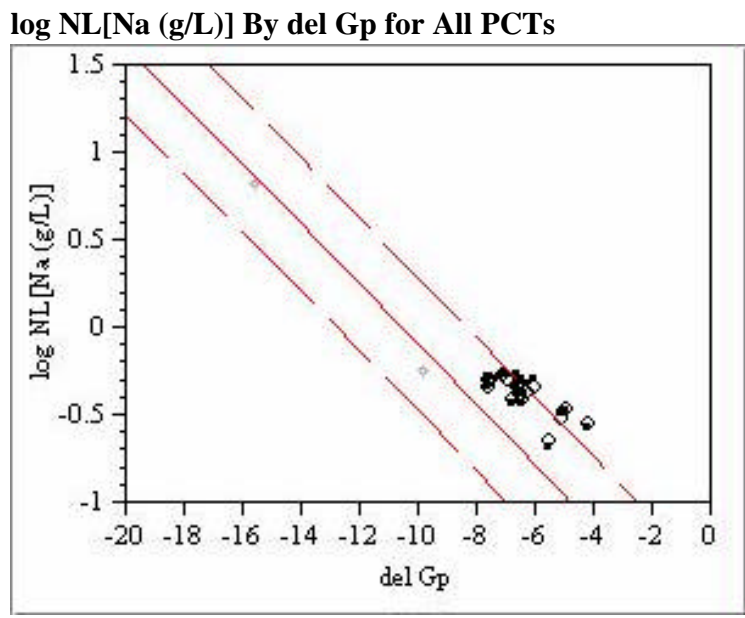

$\log$ NL[Na (g/L)] By del Gp for Screened PCTs

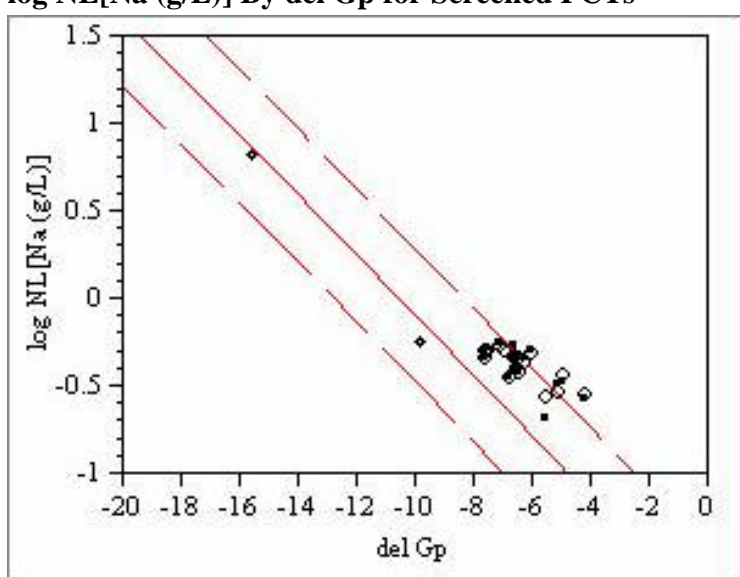

$\log$ NL[Na (g/L)] By del Gp for Valid PCTs

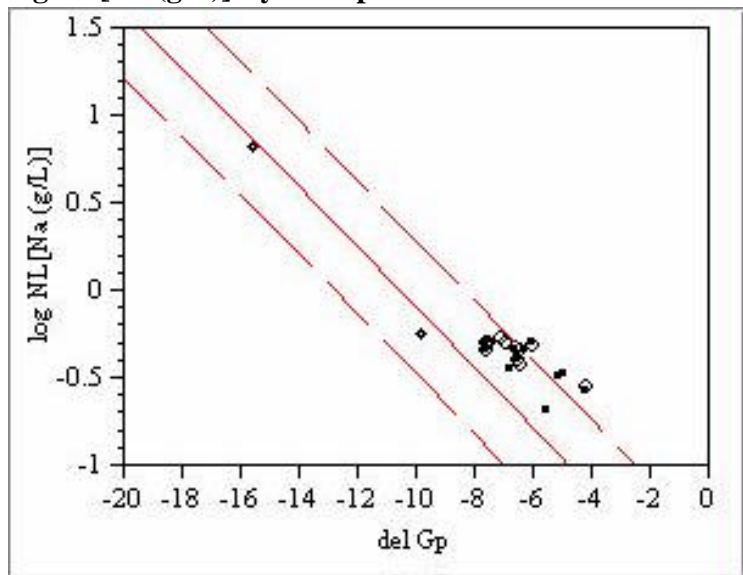

$\log$ NL[Si (g/L)] By del Gp for All PCTs

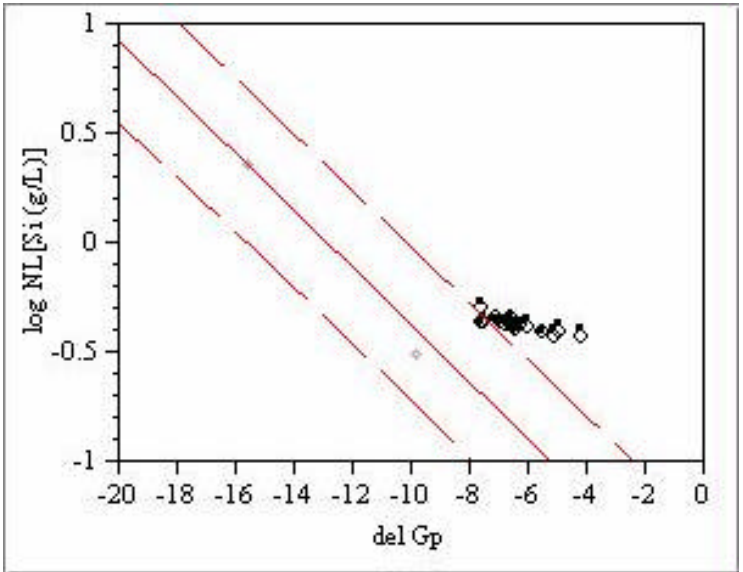

$\log$ NL[Si (g/L)] By del Gp for Screened PCTs

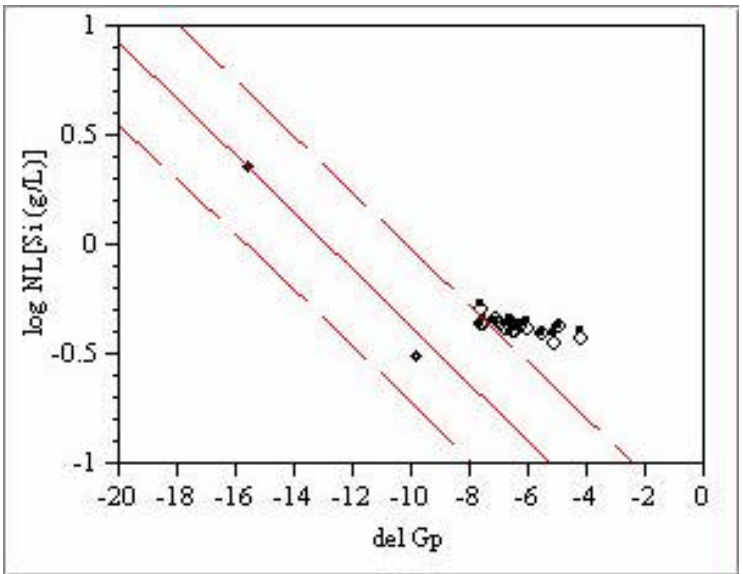

$\log$ NL[Si (g/L)] By del Gp for Valid PCTs

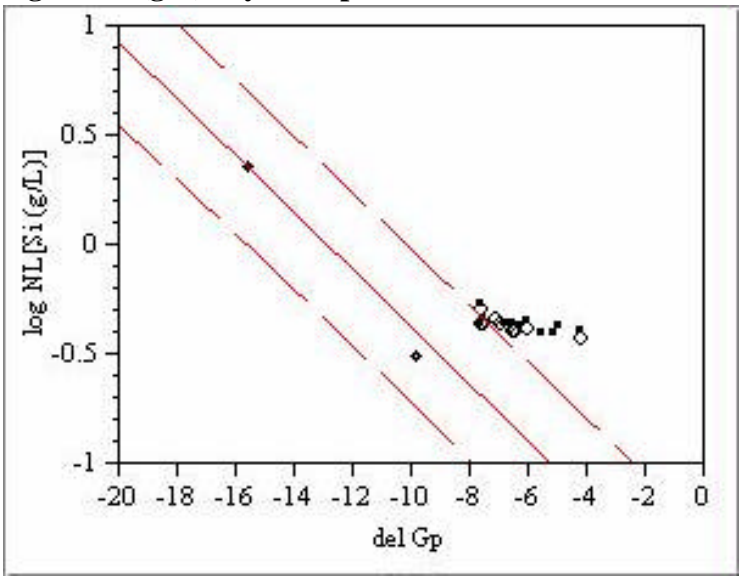


Exhibit A.6: Durability Model Predictions versus PCT Results Based Upon Targeted Glass Compositions(continued)

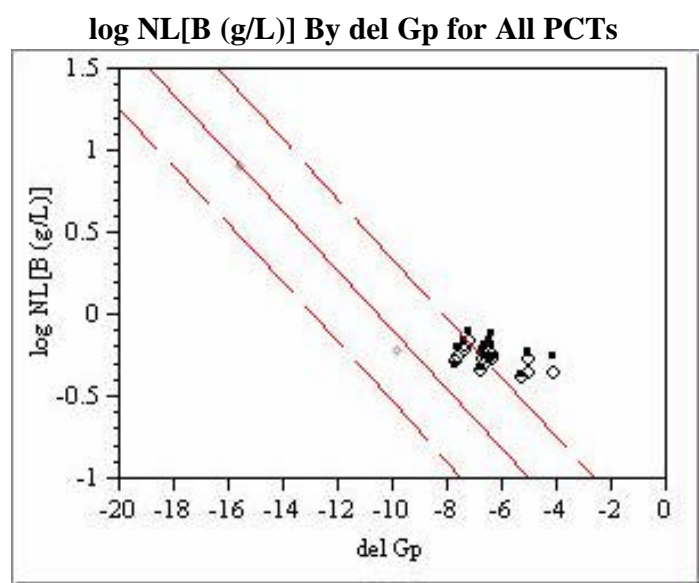

$\log$ NL[B (g/L)] By del Gp for Screened PCTs

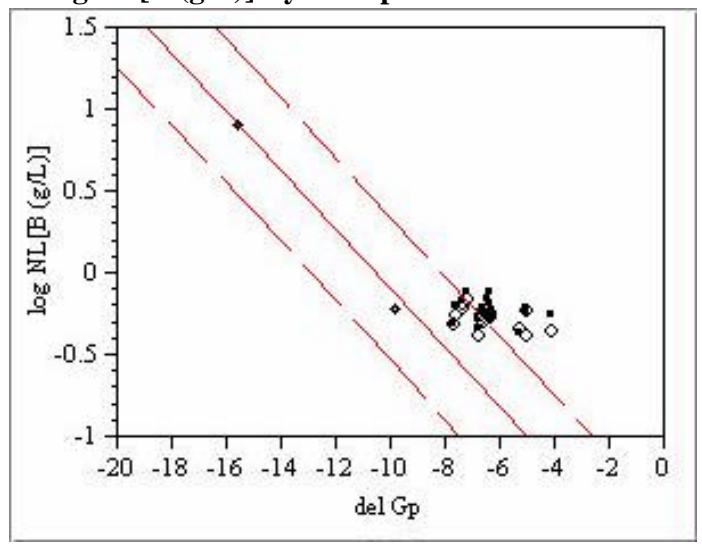

$\log$ NL[B (g/L)] By del Gp for Valid PCTs

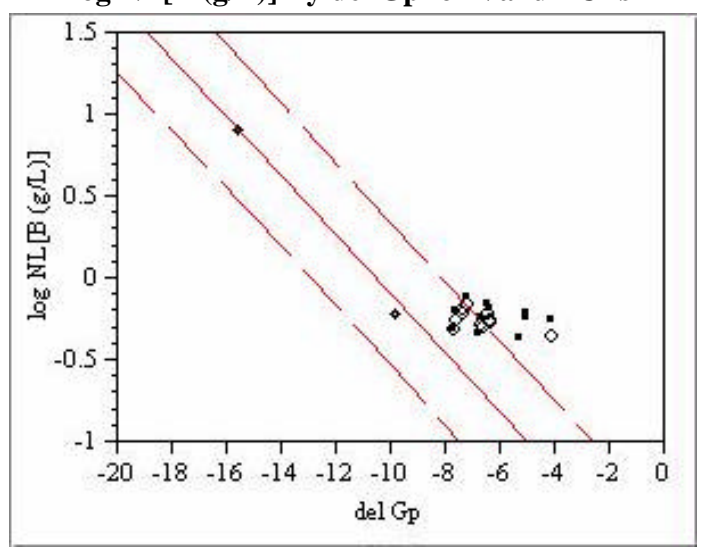

$\log$ NL[Li (g/L)] By del Gp for All PCTs

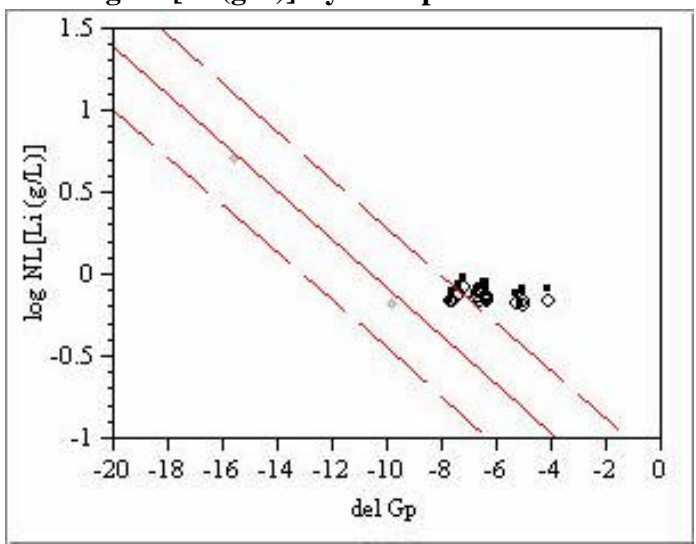

$\log$ NL[Li (g/L)] By del Gp for Screened PCTs

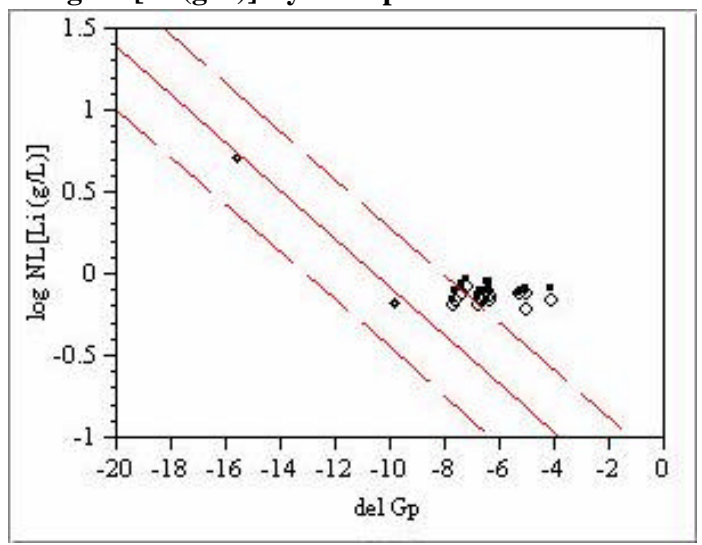

$\log \mathrm{NL}[\mathrm{Li}(\mathrm{g} / \mathrm{L})]$ By del Gp for Valid PCTs

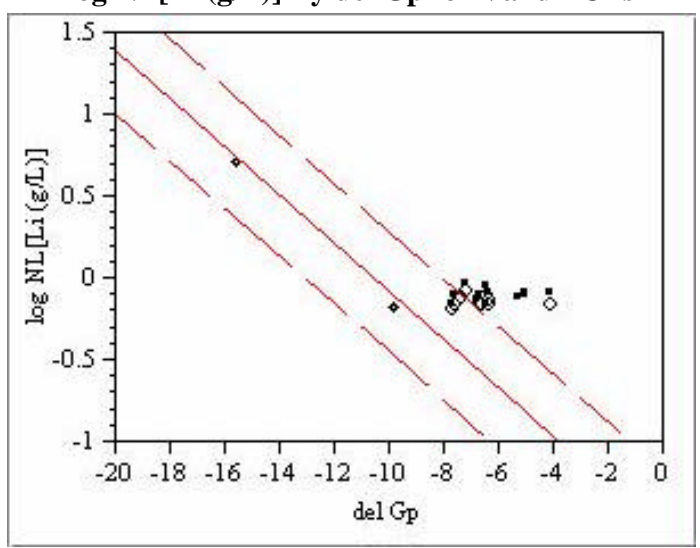


WSRC-TR-2001-00125

Revision 0

Exhibit A.6: Durability Model Predictions versus PCT Results

Based Upon Targeted Glass Compositions (continued)

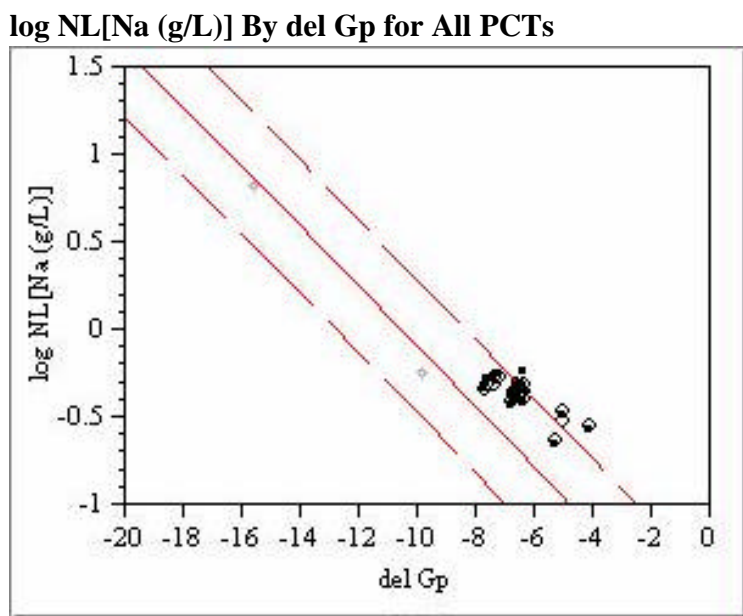

$\log$ NL[Na (g/L)] By del Gp for Screened PCTs

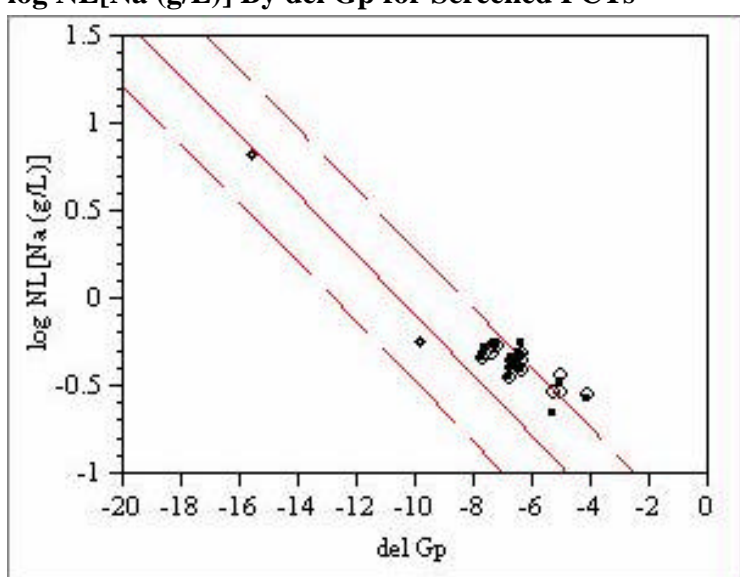

$\log$ NL[Na (g/L)] By del Gp for Valid PCTs

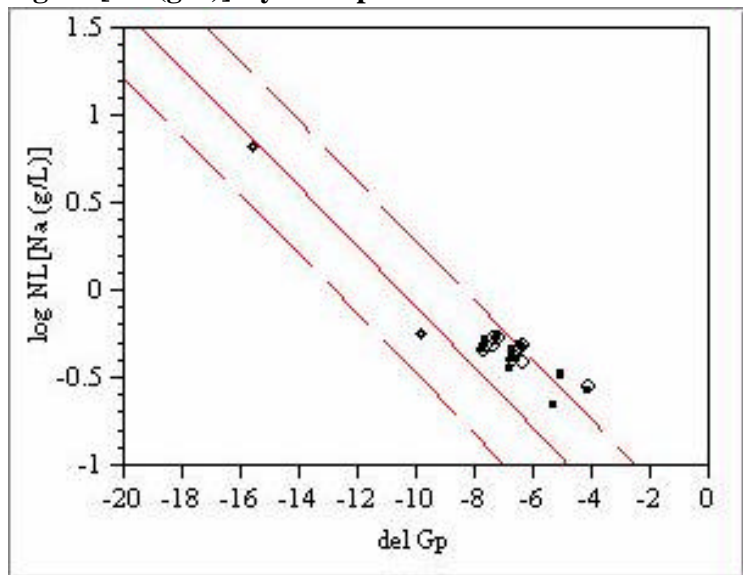

$\log$ NL[Si (g/L)] By del Gp for All PCTs

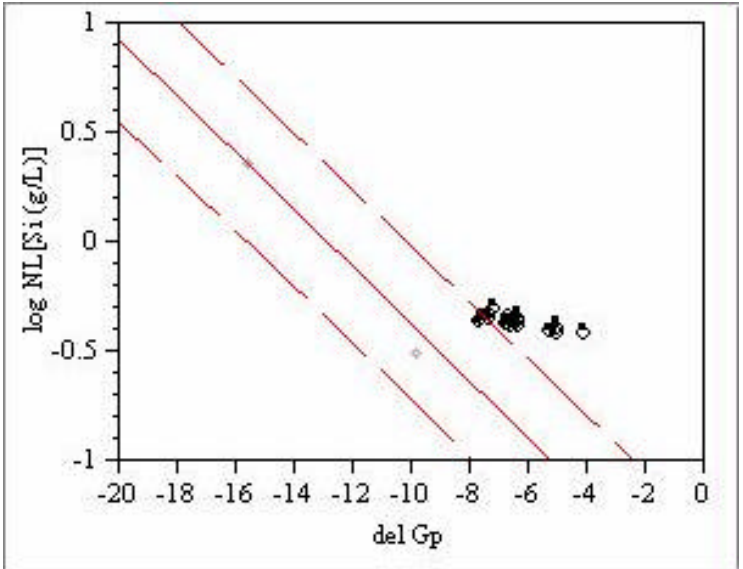

$\log$ NL[Si (g/L)] By del Gp for Screened PCTs

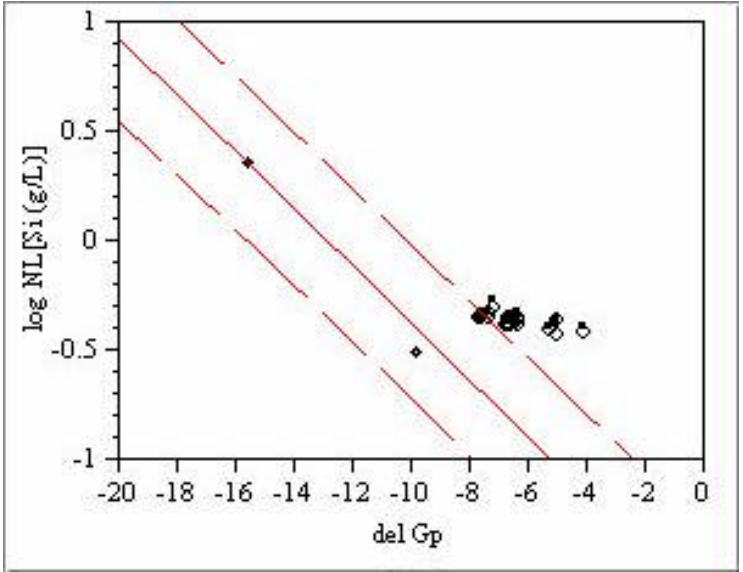

$\log$ NL[Si (g/L)] By del Gp for Valid PCTs

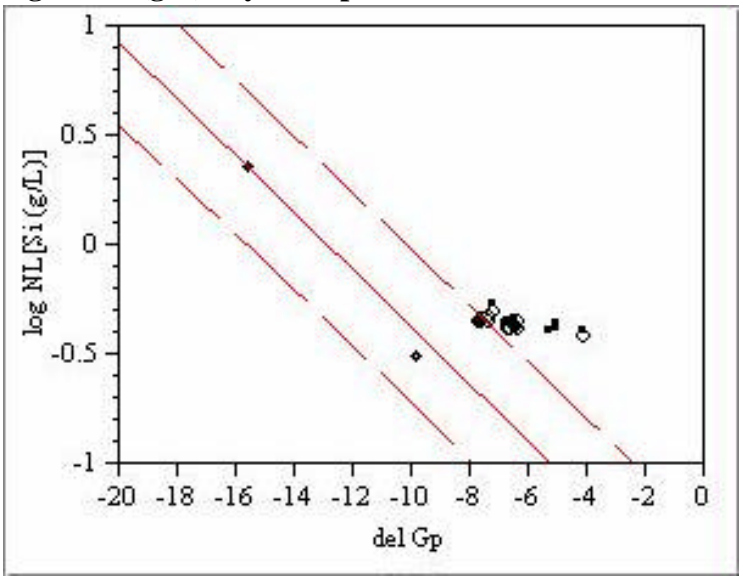


Revision 0

Exhibit A.7: Paired Comparisons of Quenched versus Centerline Cooled PCTs

Using All of the Leachate Concentrations, Using Only Those Screened For Water Loss Problems, and Using Only Valid PCTs

\section{All of the PCT Results}

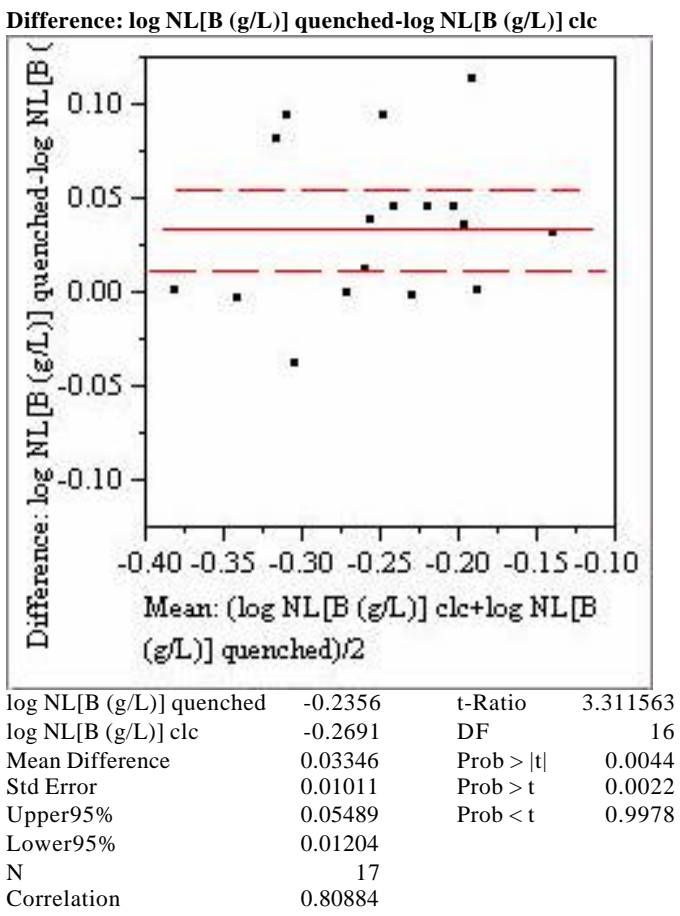

Only Results for PCTs Passing

Water Loss (i.e. Screened)

Difference: $\log N L[B(g / L)]$ quenched-log $N L[B(g / L)]$ clc

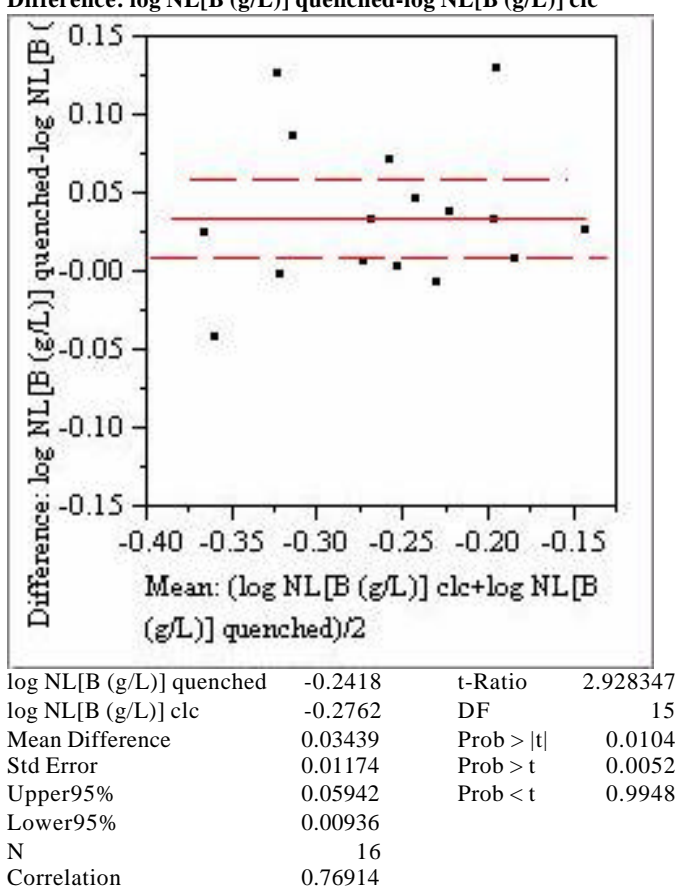

Valid PCTs Only

(i.e., those with at least 2 of the triplicates $\mathrm{OK}$ ).

Difference: $\log N L[B(g / L)]$ quenched-log NL[B (g/L)] clc

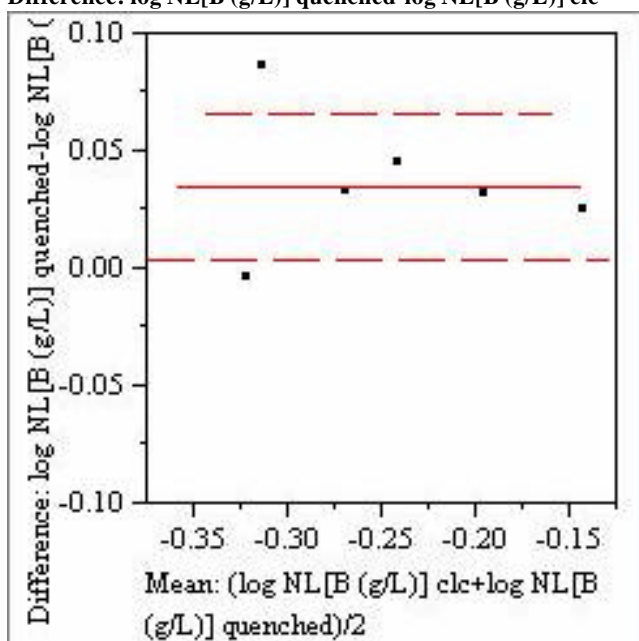

$\log \mathrm{NL}[\mathrm{B}(\mathrm{g} / \mathrm{L})]$ quenched $\quad-0.2294 \quad$ t-Ratio 2.94166 $\log \mathrm{NL}[\mathrm{B}(\mathrm{g} / \mathrm{L})]$ clc $\quad-0.2644 \quad 0.03501$ Mean Difference 0.03501 $\begin{array}{lll} & 0.0322 \\ \text { Prob }>|t| & 0.0161\end{array}$ 0.0656 Upper95\% Lower95\% $\mathrm{N}$ 0.00442

0.91725 
Exhibit A.7: Paired Comparisons of Quenched versus Centerline Cooled PCTs

Using All of the Leachate Concentrations and Using Only Those Screened For Water Loss Problems (continued)

\section{All of the PCT Results}

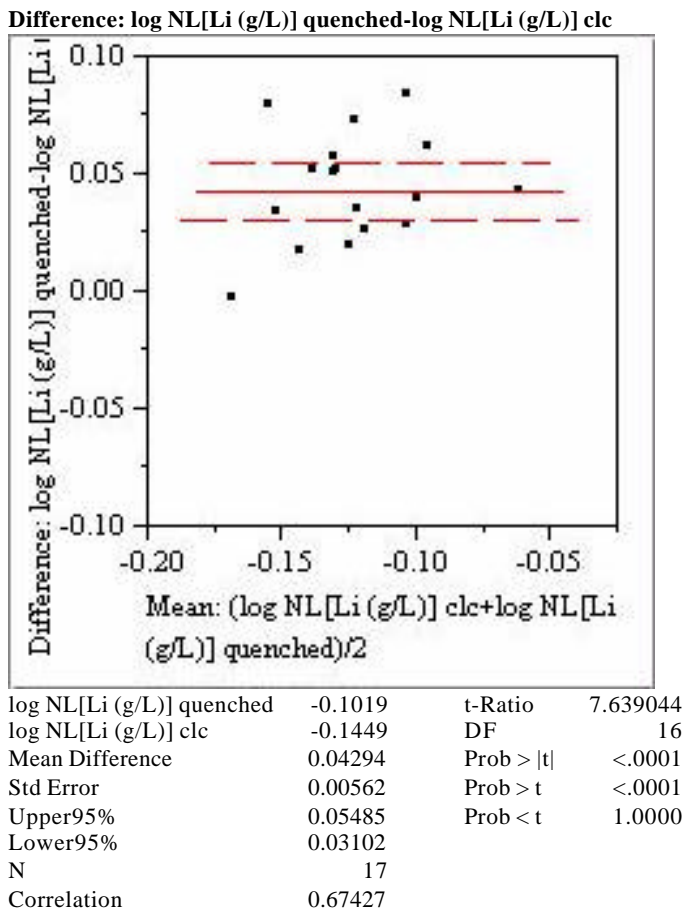

Only Results for PCTs Passing

Water Loss (i.e. Screened)

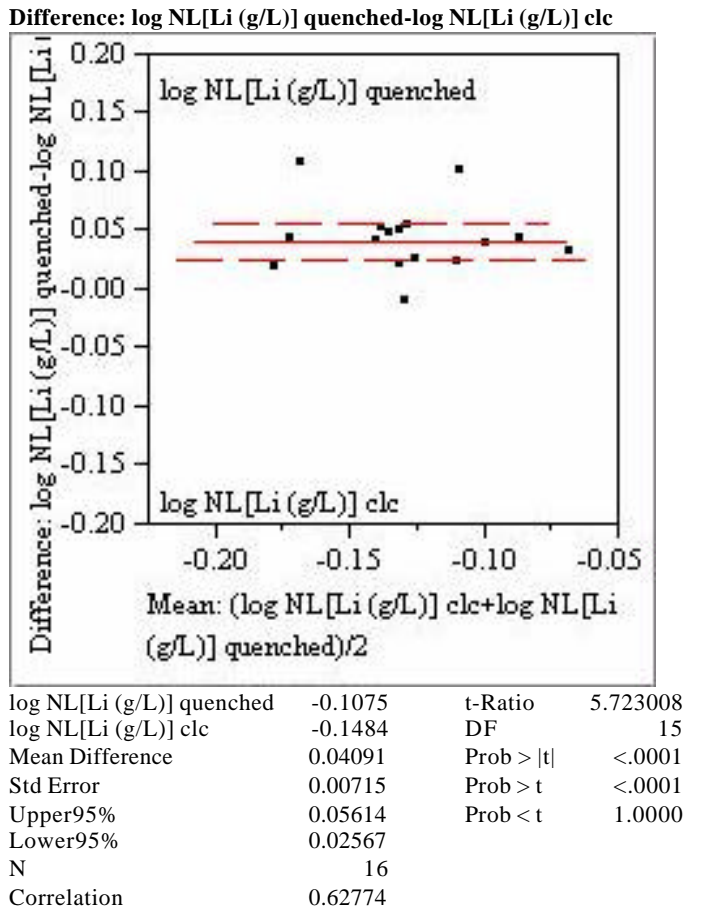

\section{Valid PCTs Only}

(i.e., those with at least 2 of the triplicates $\mathrm{OK}$ ).

Difference: $\log \mathrm{NL}[\mathrm{Li}(\mathrm{g} / \mathrm{L})]$ quenched-log $\mathrm{NL}[\mathrm{Li}(\mathrm{g} / \mathrm{L})] \mathrm{clc}$

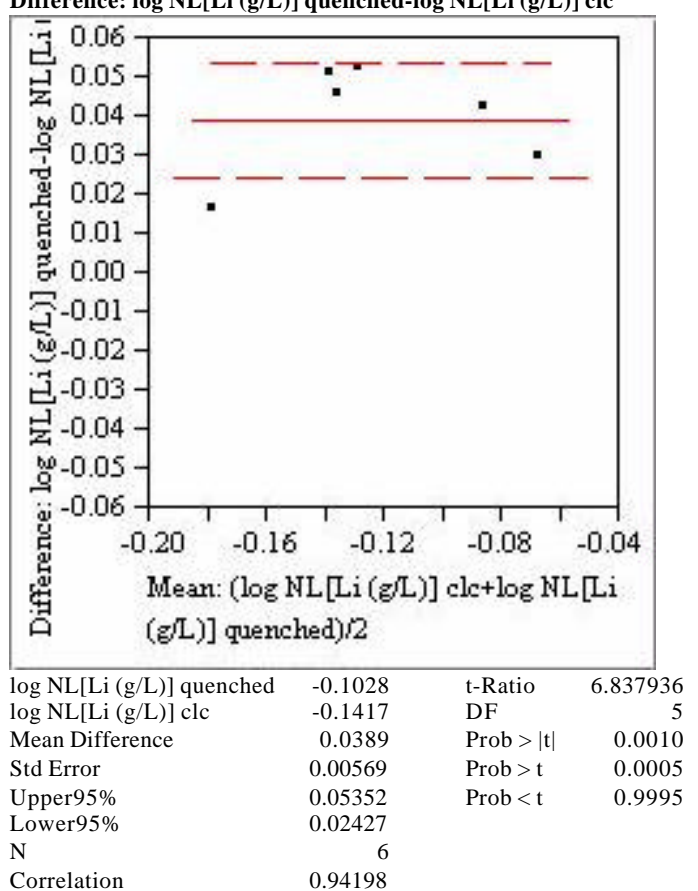


Exhibit A.7: Paired Comparisons of Quenched versus Centerline Cooled PCTs

Using All of the Leachate Concentrations and Using Only Those Screened For Water Loss Problems (continued)

\section{All of the PCT Results}

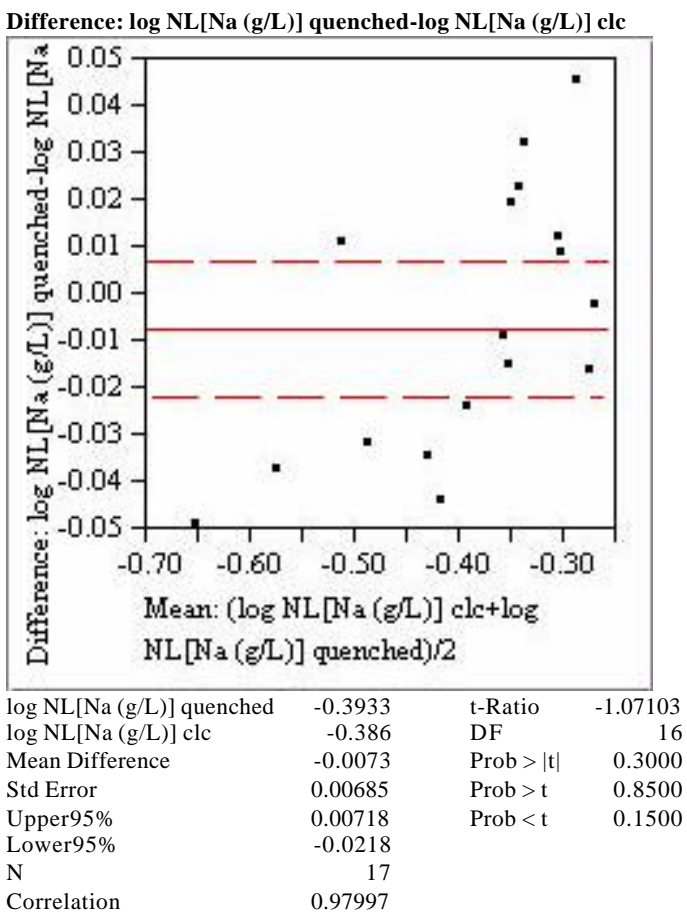

Only Results for PCTs Passing

Water Loss (i.e. Screened)

Difference: $\log \mathrm{NL}[\mathrm{Na}(\mathrm{g} / \mathrm{L})]$ quenched-log NL[Na $(\mathrm{g} / \mathrm{L})]$ clc

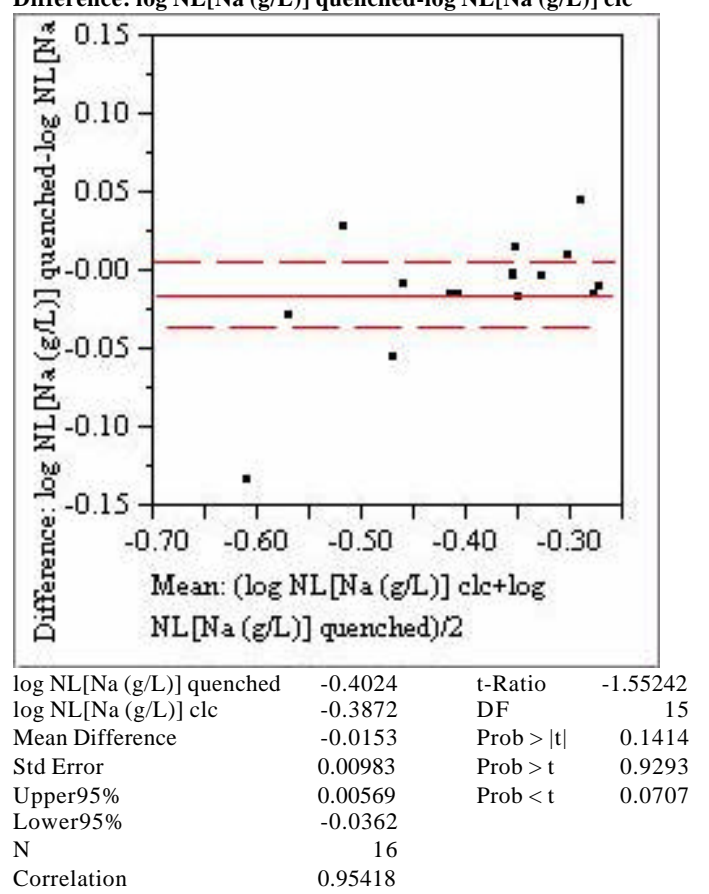

Valid PCTs Only

(i.e., those with at least 2 of the triplicates $O K$ ).

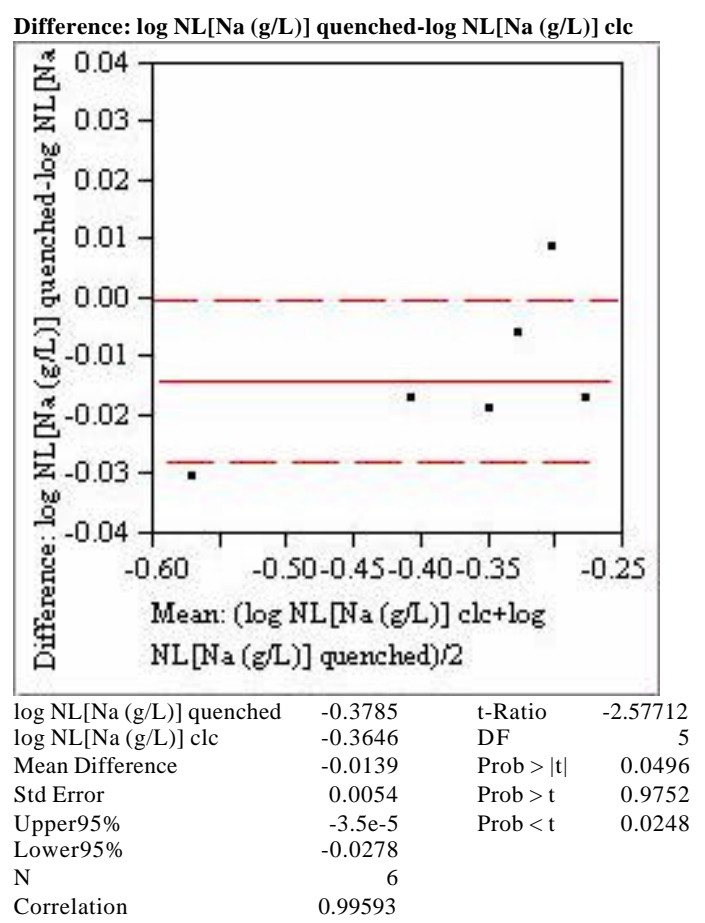


Exhibit A.7: Paired Comparisons of Quenched versus Centerline Cooled PCTs

Using All of the Leachate Concentrations and Using Only Those Screened For Water Loss Problems (continued)

\section{All of the PCT Results}

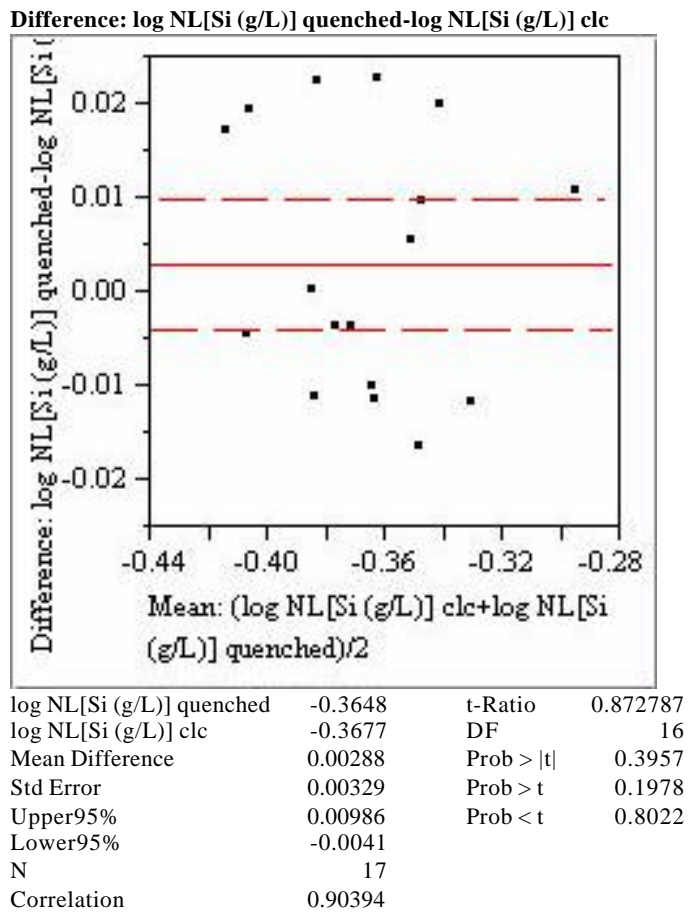

Only Results for PCTs Passing

Water Loss (i.e. Screened)

Difference: $\log \mathrm{NL}[\mathrm{Si}(\mathrm{g} / \mathrm{L})]$ quenched-log $\mathrm{NL}[\mathrm{Si}(\mathrm{g} / \mathrm{L})]$ clc

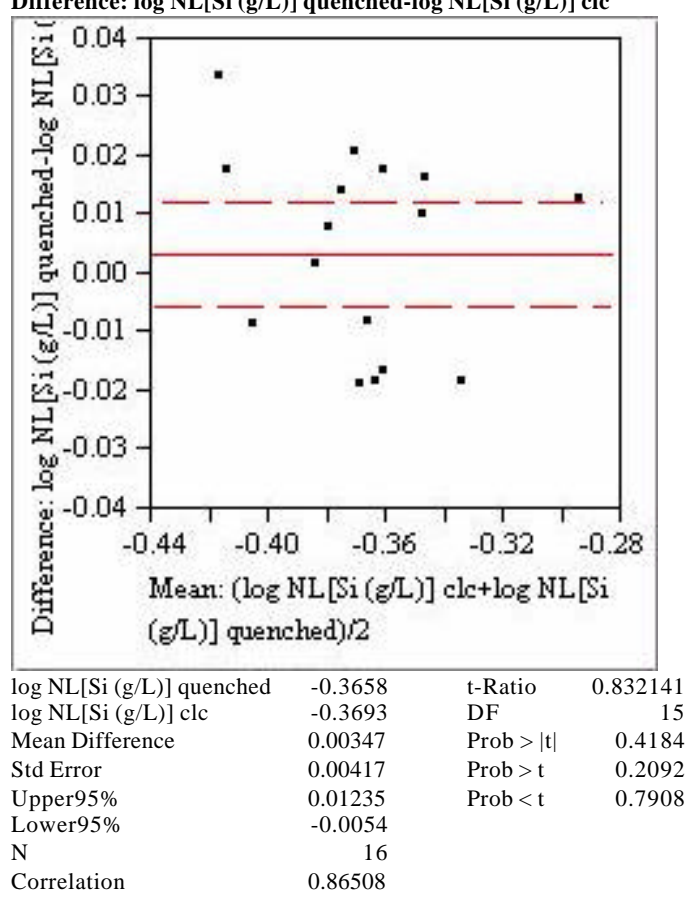

Valid PCTs Only

(i.e., those with at least 2 of the triplicates OK).

Difference: $\log \mathrm{NL}[\mathrm{Si}(\mathrm{g} / \mathrm{L})]$ quenched-log NL[Si (g/L)] clc

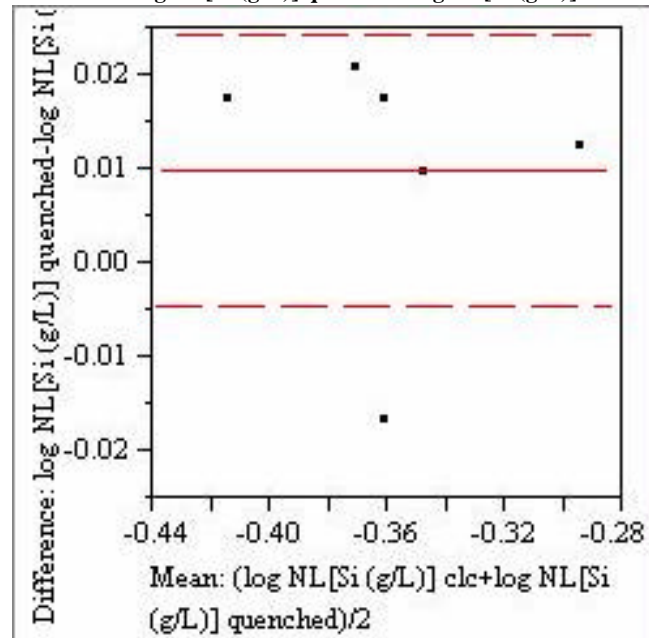

N N $[\mathrm{Si}(\mathrm{g} / \mathrm{L})] \mathrm{gue}$ $\log \mathrm{NL}[\mathrm{Si}(\mathrm{g} / \mathrm{L})] \mathrm{cuenched}-0.3526$ $\log \mathrm{NL}[\mathrm{Si}(\mathrm{g} / \mathrm{L})] \mathrm{Clc}-0.3625$ $\begin{array}{ll}\text { Mean Difference } & 0.00987 \\ \text { Std Error } & 0.00564\end{array}$

Std Error

Upper $95 \%$

$\mathrm{N}$

Correlation

0.93896 
This page intentionally left blank. 


\section{Distribution}

SRS

J. L. Barnes, 704-3N

N. E. Bibler, 773-A

D. F. Bickford, 773-43A

K. G. Brown, 773-43A

J. T. Carter, 704-3N

J. J. Connelly, 773-41A

A. D. Cozzi, 77-43A

D. A. Crowley, 773-43A

R. E. Edwards, 704-25S

T. B. Edwards, 773-42A

H. H. Elder, 704-S

S. D. Fink, 773-A

R. C. Fowler, 704-196N

J. R. Harbour, 773-43A

H. D. Harmon, 704-3N

E. W. Holtzscheiter, 773-A

R. A. Jacobs, 704-3N

C. M. Jantzen, 773-A

R. T. Jones, 704-3N

D. P. Lambert, 704-1T

S. L. Marra, 704-1T

D. B. Moore-Shedrow, 773-A

L. M. Papouchado, 773-A

D. K. Peeler, 773-43A

J. A. Pike, $704-3 \mathrm{~N}$

S. N. Schlata, 704-3N

R. F. Schumacher, 773-43A

F. G. Smith, 773-42A

M. E. Smith, 773-43A

R. H. Spires, 773-A

T. K. Snyder, 704-1T

W. L. Tamosaitis, 773-A

R. C. Tuckfield, 773-43A

R. J. Workman, 773-A

TIM (4 copies), 703-43A

\section{DOE-SR}

J. W. McCullough, Jr., 704-3N

J. M. Reynolds, II, 704-S

P. C. Suggs, 704-196N
Pacific Northwest National Laboratory PO Box 999, MSIN

Richland, WA 99352

Attn: D. W. Wester, P7-25 